Cos

\title{
Mapping gravitational-wave backgrounds using methods from CMB analysis: Application to pulsar timing arrays
}

\author{
Jonathan Gair \\ Institute of Astronomy, University of Cambridge, Madingley Road, Cambridge CB3 OHA, United Kingdom \\ Joseph D. Romano \\ Department of Physics and Astronomy and Center for Gravitational-Wave Astronomy, \\ University of Texas at Brownsville, Brownsville, Texas 78520, USA \\ Stephen Taylor \\ Institute of Astronomy, University of Cambridge, Madingley Road, Cambridge CB3 OHA, United Kingdom
}

Chiara M. F. Mingarelli

School of Physics and Astronomy, University of Birmingham, Edgbaston, Birmingham B15 2TT, United Kingdom; Max Planck Institute for Radio Astronomy, Auf dem Hügel 69, D-53121 Bonn, Germany, and Theoretical Astrophysics, California Institute of Technology, 1200 E California Blvd., M/C 350-17, Pasadena, California 91125, USA

(Received 17 June 2014; published 1 October 2014)

\begin{abstract}
We describe an alternative approach to the analysis of gravitational-wave backgrounds, based on the formalism used to characterize the polarization of the cosmic microwave background. In contrast to standard analyses, this approach makes no assumptions about the nature of the background and so has the potential to reveal much more about the physical processes that generated it. An arbitrary background can be decomposed into modes whose angular dependence on the sky is given by gradients and curls of spherical harmonics. We derive the pulsar timing overlap reduction functions for the individual modes, which are given by simple combinations of spherical harmonics evaluated at the pulsar locations. We show how these can be used to recover the components of an arbitrary background, giving explicit results for both isotropic and anisotropic uncorrelated backgrounds. We also find that the response of a pulsar timing array to curl modes is identically zero, so half of the gravitational-wave sky will never be observed using pulsar timing, no matter how many pulsars are included in the array. An isotropic, unpolarized and uncorrelated background can be accurately represented using only three modes, and so a search of this type will be only slightly more complicated than the standard cross-correlation search using the Hellings and Downs overlap reduction function. However, by measuring the components of individual modes of the background and checking for consistency with isotropy, this approach has the potential to reveal much more information. Each individual mode on its own describes a background that is correlated between different points on the sky. A measurement of the components that indicates the presence of correlations in the background on large angular scales would suggest startling new physics.
\end{abstract}

DOI: 10.1103/PhysRevD.90.082001

PACS numbers: 04.80.Nn, 04.30.Db, 07.05.Kf, 95.55.Ym

\section{INTRODUCTION}

Near-future detections of gravitational waves (GWs) will open a new window onto the cosmos by allowing astrophysical and cosmological phenomena that generate only weak or difficult-to-detect electromagnetic signatures to be probed for the first time and with an unprecedented precision. Within the next several years a global network of advanced kilometer-scale laser interferometers will come online, providing insights into stellar-mass compact binary systems and stochastic gravitational-wave backgrounds in the $\mathrm{kHz}$ band [1-4]. In 20 years, the launch of a $\sim 10^{9} \mathrm{~m}$ arm-length space-based laser interferometer will allow precision tests of fundamental physics and perform detailed demographic studies of massive black holes throughout the Universe [5].

Concurrently with these efforts are dedicated programs observing the regular pulsed emission from ensembles of Galactic millisecond pulsars with the aim of detecting and characterizing nanohertz gravitational waves [6-9]. The long-term stability of integrated pulse profiles allows incredibly accurate models of the time of arrival (TOA) of pulses to be constructed and enables these pulsars to be used as standard clocks in the sky. Potential gravitationalwave targets in the $\mathrm{nHz}$ band are single resolvable sources [e.g., chirping supermassive black-hole (SMBH) binaries [10-12] or cosmic-string bursts [13-15]] and stochastic backgrounds from the superposition of many inspiraling 
SMBH binary systems [16-18], decaying cosmic-string networks [19-22], or even backgrounds of primordial origin $[23,24]$.

A pulsar timing array (PTA) can be thought of as a galactic-scale gravitational-wave detector [25]. When a gravitational wave transits the Earth-pulsar line of sight, it creates a perturbation in the intervening metric, causing a change in the proper separation, which manifests as a redshift in the pulse frequency [26-29]. Standard timing models only factor in deterministic influences to the TOAs, such that a subtraction of modeled TOAs from the raw observations will result in a stream of timing residuals, which encode the influence of gravitational waves along with stochastic noise processes. A PTA allows one to crosscorrelate the residuals from many pulsars, leveraging the common influence of a gravitational-wave background against undesirable, uncorrelated noise processes.

In fact, for a Gaussian-stationary, isotropic, unpolarized stochastic background composed of plus/cross gravitationalwave polarization states, the cross-correlation of timing residuals is a unique smoking-gun signature of the background's presence, and it depends only on the angular separation between pulsars on the sky: this is the famous Hellings and Downs curve [30]. Backgrounds composed of non-Einsteinian polarization states [31,32], or influenced by nonzero graviton mass [33], will induce different correlation signatures, as will anisotropy in the background's energy density [34,35], where the signature will contain rich information on the distribution of gravitational-wave power with respect to the position of pulsars on the sky.

Each of these standard analyses assumes a model describing the nature of the background and then tries to measure a small number of model parameters. For an isotropic, unpolarized and uncorrelated background there is just one measurable parameter, which is the amplitude of the background. While such analyses are optimal for the type of background being modeled, they will not be as sensitive to alternative models and will not indicate whether the model is correct. In this paper, we describe how pulsar timing residuals can be used instead to construct a map of the gravitational-wave background that makes no assumptions about its nature. The properties of the observed background can be checked for consistency with any particular model, e.g., to what extent it is isotropic, unpolarized and uncorrelated, but this approach has the potential to reveal much more, since we will extract all of the information that can be determined about the background by a given pulsar timing array. This information will not only tell us which out of our current models provides the best description of the background, but will clearly indicate if none of those models are accurate and therefore that new physical models of the background are required.

The polarization of the cosmic microwave background (CMB), which has two independent components, can be represented as a transverse traceless tensor field on the sky
[36]. In the analysis of CMB data, the polarization field is represented as a superposition of gradients and curls of spherical harmonics, and $\mathrm{CMB}$ measurements attempt to determine the individual components of those modes. A gravitational-wave background is also a transverse traceless tensor field on the sky, and so the same formalism can be applied to the analysis of a gravitational-wave background. It is this that we describe in this paper. That the CMB approach can be readily applied to gravitational waves is most easily seen from the fact that the gradients and curls of spherical harmonics can also be written as the real and imaginary parts of spin- \pm 2 spin-weighted spherical harmonics, which are widely used to decompose the gravitational-wave emission from a source [37].

Any gravitational-wave background can be decomposed as a sum of gradient and curl modes. The components of this decomposition are the expansion coefficients of the metric perturbation in terms of the gradient and curl spherical harmonics; see Eq. (10). The signature that arises in the cross-correlation of the timing residuals of pairs of pulsars in a PTA can therefore be computed as a sum of the cross-correlation curves (overlap reduction functions) of each mode. For an unpolarized statistically isotropic background the overlap reduction functions for the individual modes are just Legendre polynomials, and the Hellings and Downs curve can be recovered straightforwardly as a superposition of these. Three modes are sufficient to represent the Hellings and Downs correlation for reasonable assumptions about the PTA, so applying this formalism to an isotropic, unpolarized and uncorrelated background will not be much more computationally challenging than the standard analysis.

The overlap reduction functions for individual modes can also be computed for anisotropic backgrounds. For pulsar timing arrays, the resulting expression is relatively simple since the response of a pulsar to curl modes is identically zero, while the response to a gradient mode is proportional to the corresponding spherical harmonic evaluated at the direction to the pulsar. For anisotropic, unpolarized and uncorrelated backgrounds, the integral expressions for the spherical harmonic components of the overlap reduction function can be evaluated analytically, allowing us to extend the results given for quadrupole and lower backgrounds in [34].

It is also relatively straightforward to reconstruct a map of the gravitational-wave sky for that part of the background spanned by the gradient modes visible to a PTA, and we describe how this can be done. For a PTA consisting of $N$ pulsars, at any given frequency we make two measurements - an amplitude and a phase-with each pulsar. Since PTAs are static, the response function is frequency independent, and we would therefore not expect to be able to measure more than $2 N$ real components of the background. The fact that PTAs are sensitive to only $2 \mathrm{~N}$ components of the background is consistent with recent unpublished results by Cornish and van Haasteren (private 
communication). We describe how we can recover these $N$ complex combinations of gradient mode components and which components we expect to measure most accurately (those for the low- $l$ modes). In practice, we can either restrict our mapping search to fewer than $N$ low- $l$ modes or use singular-value decomposition (SVD) of the mapping matrix to determine the $N$ linear combinations to which the array is sensitive. Since we make no assumptions about the properties of the underlying background in this analysis, we can interpret the map that we obtain in terms of its implications for fundamental physics, as described below. To characterize an isotropic, unpolarized and uncorrelated background we need to reach an angular resolution of $l_{\max } \sim 4$, which requires 21 pulsars, well within reach of current PTA efforts. To reach the angular resolution at which we expect to resolve individual sources with a PTA we must probe $l_{\max } \sim 10$, which will require $\sim 100$ pulsars. This should be achievable with the square-kilometer array (SKA) [38].

In our approach, each individual mode used in the decomposition describes a background that is correlated between different points on the sky. By this we will mean a correlation in the gravitational radiation coming from different angular directions, which is different from the correlation between the pulsar responses, present for all types of backgrounds. It is also different from spatial correlations that may exist between the metric perturbations evaluated at different locations in space. A background that is spatially homogeneous and isotropic can have spatial correlations provided the correlations depend only on the distance $|\vec{x}-\vec{y}|$ between any two points $\vec{x}$ and $\vec{y}$, and any background of this form will be uncorrelated in Fourier (angle) space. We focus on angular correlations because we will measure the gravitational-wave background at a single point only and therefore cannot compute spatial correlations from our data. Assumptions about the presence or absence of spatial correlations are needed to compute the statistical properties of a background in any particular physical model, but here we will focus only on a measurement of the background, and so the angular correlation properties are the most important.

The gravitational-wave background in the pulsar timing band is most likely generated by a superposition of emission from many individual astrophysical sources. Such a background will not show angular correlations between different sky locations, but would show anisotropy indicative of the spatial distribution of sources contributing to the background. A background of cosmological origin could in principle show angular correlations on some scale, and the spectrum of modes present will be characteristic of the quantum fluctuations that produced it. However, there are no mechanisms currently known that would generate such correlations in the nanohertz frequency band. Nonetheless, the power of the analysis described here is that it can represent any background and it makes no assumptions about the correlation properties or isotropy. It will allow us to derive a map of the background, free from model assumptions, that will encode all of the details about the underlying physical processes that produced the background and that are possible to deduce from our observations. If the map indicates the presence of correlated emission or significant anisotropy, it will be a startling and profound result, pointing to either unmodeled physics in the early Universe or an unknown systematic affecting the timing data. In either case, the result would be of great significance.

This paper is organized as follows: In Sec. II we describe the general formalism, which is based on that used to characterize $\mathrm{CMB}$ polarization and can be used to describe arbitrary gravitational-wave backgrounds. We include a description of the basis functions used to expand the backgrounds, and we give definitions of the response functions and overlap reduction functions for arbitrary gravitational-wave detectors. In Sec. III we specialize to the case of PTAs, deriving the overlap reduction function for an unpolarized statistically isotropic background, and show how the Hellings and Downs curve for an isotropic, unpolarized and uncorrelated background is well approximated by a combination of the first three modes, $l=2,3,4$. We show that the response of a pulsar to the curl modes of a gravitational-wave background is identically zero, while the response to an individual gradient mode is simply proportional to the corresponding spherical harmonic evaluated at the direction to the pulsar. We also demonstrate how the formalism can be used by recovering the coefficients of the expansion from a simulated pulsar-timing data set. In Sec. IV we compute the overlap reduction functions needed to represent arbitrary anisotropic backgrounds, giving explicit expressions for a PTA. In Sec. V we discuss how one can reconstruct a map of the gravitational-wave sky in terms of the gradient components visible to a PTA. We show that an $N$-pulsar array can measure $N$ (complex) combinations of the gradient components of the background, but is blind to the curl component, irrespective of the value of $N$. Finally, in Sec. VI we summarize the results and discuss some of the implications if a measurement of these parameters is made that is indicative of significant correlations in the background.

We also include several appendixes: Appendixes A and $\mathrm{B}$ contain useful definitions and identities for spinweighted spherical harmonics and associated Legendre functions and Legendre polynomials, respectively. In Appendix $\mathrm{C}$, we calculate the oscillatory behavior of the pulsar term for an isotropic, unpolarized and uncorrelated stochastic background and show that it is negligible. In Appendix D, we derive the grad and curl responses for a static interferometer and find that the curl response is zero, similar to that for a PTA. In Appendix E, we derive analytic expressions for the spherical harmonic components of the overlap reduction function for anisotropic, unpolarized and 
uncorrelated backgrounds for all values of $l$ and $m$, extending the analytical results of [34].

\section{GENERAL FORMALISM}

The gravitational-wave field is a symmetric transversetraceless tensor field, with two independent polarization states, $h_{+}$and $h_{\times}$, which transform under rotations of the polarization axes defined at each point on the sky [39]. In the analysis of the $\mathrm{CMB}$, polarization is characterized by a two-dimensional, symmetric and trace-free matrix, which is analogous to the symmetric transverse-traceless metric perturbations describing a general gravitational-wave field. Therefore, our analysis will closely parallel the treatment of polarization in analyses of the CMB; see, e.g., [36,40].

\section{A. Gradient and curl spherical harmonics}

Any symmetric trace-free rank-two tensor field on the two-sphere $S^{2}$ can be written as the sum of the "gradient" of a scalar field $A(\hat{k})$

$$
A_{; a b}-\frac{1}{2} g_{a b} A_{; c}{ }^{c}
$$

plus the "curl" of another scalar field $B(\hat{k})$

$$
\frac{1}{2}\left(B_{; a c} \epsilon_{b}^{c}+B_{; b c} \epsilon_{a}^{c}\right)
$$

where a semicolon denotes covariant differentiation, $g_{a b}$ is the metric tensor on the sphere, and $\epsilon_{a b}$ is the Levi-Cività antisymmetric tensor

$$
\epsilon_{a b}=\sqrt{g}\left(\begin{array}{cc}
0 & 1 \\
-1 & 0
\end{array}\right)
$$

Following standard practice, we use the metric tensor $g_{a b}$ and its inverse $g^{a b}$ to "lower" and "raise" tensor indicese.g., $\epsilon_{b}^{c} \equiv g^{c a} \epsilon_{a b}$. In standard spherical coordinates $(\theta, \phi)$,

$$
g_{a b}=\left(\begin{array}{cc}
1 & 0 \\
0 & \sin ^{2} \theta
\end{array}\right), \quad \sqrt{g}=\sin \theta .
$$

Since any scalar field on the two-sphere can be written as a sum of spherical harmonics, $Y_{l m}(\hat{k})$, it follows that any symmetric trace-free rank-two tensor field can be written as a sum of gradients and curls of spherical harmonics $[36,41]$.

Defining the gradient and curl spherical harmonics for $l \geq 2$ by

$$
\begin{aligned}
& Y_{(l m) a b}^{G}=N_{l}\left(Y_{(l m) ; a b}-\frac{1}{2} g_{a b} Y_{(l m) ; c} c\right), \\
& Y_{(l m) a b}^{C}=\frac{N_{l}}{2}\left(Y_{(l m) ; a c} \epsilon_{b}^{c}+Y_{(l m) ; b c} \epsilon_{a}^{c}\right),
\end{aligned}
$$

where

$$
N_{l}=\sqrt{\frac{2(l-2) !}{(l+2) !}},
$$

it follows that

$$
\begin{aligned}
& \int_{S^{2}} \mathrm{~d}^{2} \Omega_{\hat{k}} Y_{(l m) a b}^{G}(\hat{k}) Y_{\left(l^{\prime} m^{\prime}\right)}^{G} a b *(\hat{k})=\delta_{l l^{\prime}} \delta_{m m^{\prime}}, \\
& \int_{S^{2}} \mathrm{~d}^{2} \Omega_{\hat{k}} Y_{(l m) a b}^{C}(\hat{k}) Y_{\left(l^{\prime} m^{\prime}\right)}^{C} a b *(\hat{k})=\delta_{l l^{\prime}} \delta_{m m^{\prime}}, \\
& \int_{S^{2}} \mathrm{~d}^{2} \Omega_{\hat{k}} Y_{(l m) a b}^{G}(\hat{k}) Y_{\left(l^{\prime} m^{\prime}\right)}^{C} a b *(\hat{k})=0 .
\end{aligned}
$$

Note that we have adopted the notational convention used in the CMB literature, e.g., [36], by putting parentheses around multipole moment indices $l$ and $m$ to distinguish these indices from spatial tensor indices $a, b$, etc.

\section{B. Expanding the metric perturbations}

In transverse-traceless coordinates, the metric perturbations $h_{a b}(t, \vec{x})$ associated with a gravitational wave are transverse to the direction of propagation $\hat{k}$ and hence define a symmetric trace-free tensor field on the twosphere. The Fourier components $h_{a b}(f, \hat{k})$ of the field can therefore be decomposed as

$$
\begin{array}{r}
h_{a b}(f, \hat{k})=\sum_{l=2}^{\infty} \sum_{m=-l}^{l}\left[a_{(l m)}^{G}(f) Y_{(l m) a b}^{G}(\hat{k})\right. \\
\left.\quad+a_{(l m)}^{C}(f) Y_{(l m) a b}^{C}(\hat{k})\right],
\end{array}
$$

with

$$
\begin{aligned}
& a_{(l m)}^{G}(f)=\int_{S^{2}} \mathrm{~d}^{2} \Omega_{\hat{k}} h_{a b}(f, \hat{k}) Y_{(l m)}^{G} a b *(\hat{k}), \\
& a_{(l m)}^{C}(f)=\int_{S^{2}} \mathrm{~d}^{2} \Omega_{\hat{k}} h_{a b}(f, \hat{k}) Y_{(l m)}^{C} a b *(\hat{k}) .
\end{aligned}
$$

Note that the summation over $l$ starts at $l=2$ and not at $l=0$, as would be the case if we were expanding a scalar function on the sphere in terms of ordinary (i.e., undifferentiated) spherical harmonics $Y_{l m}(\hat{k})$. In what follows we will use the shorthand notation $\sum_{(l m)}$ for $\sum_{l=2}^{\infty} \sum_{m=-l}^{l}$. From the above definitions it follows that

$$
\begin{aligned}
& Y_{(l m) a b}^{G, C *}(\hat{k})=(-1)^{m} Y_{(l,-m) a b}^{G, C}(\hat{k}), \\
& Y_{(l m)}^{G}(-\hat{k})=(-1)^{l} Y_{(l m)}^{G}(\hat{k}), \\
& Y_{(l m)}^{C}(-\hat{k})=(-1)^{l+1} Y_{(l m)}^{C}(\hat{k}),
\end{aligned}
$$

and 


$$
\begin{aligned}
& a_{(l m)}^{G, C *}(f)=(-1)^{m} a_{(l,-m)}^{G, C}(-f), \\
& a_{(l m)}^{G}(f) \rightarrow(-1)^{l} a_{(l m)}^{G}(f), \\
& a_{(l m)}^{C}(f) \rightarrow(-1)^{l+1} a_{(l m)}^{C}(f),
\end{aligned}
$$

with respect to complex conjugation and parity (i.e., $\hat{k} \rightarrow-\hat{k}$ ) transformations. Note that the gradient modes have "electric-type" parity, while the curl modes have "magnetictype" parity. These are sometimes referred to as " $E$ modes" and " $B$ modes," respectively, in the CMB literature.
A general stochastic gravitational-wave background can be written as a superposition of plane waves having frequency $f$ and propagation direction $\hat{k}$. We assume that gravitational waves of different frequencies are uncorrelated with one another, which follows if the background is stationary with respect to time. Using the preceding decomposition, we can therefore write the metric perturbation induced by an arbitrary stochastic background in transverse-traceless coordinates as

$$
h_{a b}(t, \vec{x})=\int_{-\infty}^{\infty} \mathrm{d} f \int_{S^{2}} \mathrm{~d}^{2} \Omega_{\hat{k}}\left\{\sum_{(l m)}\left[a_{(l m)}^{G}(f) Y_{(l m) a b}^{G}(\hat{k})+a_{(l m)}^{C}(f) Y_{(l m) a b}^{C}(\hat{k})\right]\right\} e^{i 2 \pi f(t-\hat{k} \cdot \vec{x} / c)} .
$$

Introducing the usual orthogonal coordinate axes on the sky

$$
\begin{aligned}
\hat{k} & =\sin \theta \cos \phi \hat{x}+\sin \theta \sin \phi \hat{y}+\cos \theta \hat{z}=\hat{r}, \\
\hat{l} & =\cos \theta \cos \phi \hat{x}+\cos \theta \sin \phi \hat{y}-\sin \theta \hat{z}=\hat{\theta}, \\
\hat{m} & =-\sin \phi \hat{x}+\cos \phi \hat{y}=\hat{\phi},
\end{aligned}
$$

and defining two polarization tensors by

$$
\begin{aligned}
& e_{a b}^{+}(\hat{k})=\hat{l}_{a} \hat{l}_{b}-\hat{m}_{a} \hat{m}_{b}, \\
& e_{a b}^{\times}(\hat{k})=\hat{l}_{a} \hat{m}_{b}+\hat{m}_{a} \hat{l}_{b},
\end{aligned}
$$

the gradient and curl spherical harmonics can be written explicitly as [42]

$$
\begin{aligned}
& Y_{(l m) a b}^{G}(\hat{k})=\frac{N_{l}}{2}\left[W_{(l m)}(\hat{k}) e_{a b}^{+}(\hat{k})+X_{(l m)}(\hat{k}) e_{a b}^{\times}(\hat{k})\right], \\
& Y_{(l m) a b}^{C}(\hat{k})=\frac{N_{l}}{2}\left[W_{(l m)}(\hat{k}) e_{a b}^{\times}(\hat{k})-X_{(l m)}(\hat{k}) e_{a b}^{+}(\hat{k})\right],
\end{aligned}
$$

where

$$
\begin{aligned}
W_{(l m)}(\hat{k}) & =\left(\frac{\partial^{2}}{\partial \theta^{2}}-\cot \theta \frac{\partial}{\partial \theta}+\frac{m^{2}}{\sin ^{2} \theta}\right) Y_{(l m)}(\hat{k}) \\
& =\left(2 \frac{\partial^{2}}{\partial \theta^{2}}+l(l+1)\right) Y_{(l m)}(\hat{k}), \\
X_{(l m)}(\hat{k}) & =\frac{2 i m}{\sin \theta}\left(\frac{\partial}{\partial \theta}-\cot \theta\right) Y_{(l m)}(\hat{k}) .
\end{aligned}
$$

These functions are related to spin- 2 spherical harmonics $[43,44]$ through the equation

$$
{ }_{ \pm 2} Y_{(l m)}(\hat{k})=\frac{N_{l}}{\sqrt{2}}\left[W_{(l m)}(\hat{k}) \pm i X_{(l m)}(\hat{k})\right]
$$

and can be written in terms of associated Legendre functions as

$$
W_{(l m)}(\hat{k})=+2 \sqrt{\frac{2 l+1}{4 \pi} \frac{(l-m) !}{(l+m) !}} G_{(l m)}^{+}(\cos \theta) e^{i m \phi},
$$

$$
\begin{aligned}
i X_{(l m)}(\hat{k})= & -2 \sqrt{\frac{2 l+1}{4 \pi} \frac{(l-m) !}{(l+m) !}} G_{(l m)}^{-}(\cos \theta) e^{i m \phi}, \\
G_{(l m)}^{+}(\cos \theta)= & -\left(\frac{l-m^{2}}{\sin ^{2} \theta}+\frac{1}{2} l(l-1)\right) P_{l}^{m}(\cos \theta) \\
& +(l+m) \frac{\cos \theta}{\sin ^{2} \theta} P_{l-1}^{m}(\cos \theta), \\
G_{(l m)}^{-}(\cos \theta)= & \frac{m}{\sin ^{2} \theta}\left[(l-1) \cos \theta P_{l}^{m}(\cos \theta)\right. \\
& \left.-(l+m) P_{l-1}^{m}(\cos \theta)\right] .
\end{aligned}
$$

Using this explicit form for the gradient and curl spherical harmonics, Eq. (14) becomes

$$
\begin{aligned}
h_{a b}(t, \vec{x})=\int_{-\infty}^{\infty} \mathrm{d} f \int_{S^{2}} \mathrm{~d}^{2} \Omega_{\hat{k}}\left\{\sum_{(l m)} \frac{N_{l}}{2}\left(a_{(l m)}^{G}(f) W_{(l m)}(\hat{k})-a_{(l m)}^{C}(f) X_{(l m)}(\hat{k})\right) e_{a b}^{+}(\hat{k})\right. \\
\left.\quad+\frac{N_{l}}{2}\left(a_{(l m)}^{G}(f) X_{(l m)}(\hat{k})+a_{(l m)}^{C}(f) W_{(l m)}(\hat{k})\right) e_{a b}^{\times}(\hat{k})\right\} e^{i 2 \pi f(t-\hat{k} \cdot \vec{x} / c)} .
\end{aligned}
$$

In terms of the more traditional "plus" and "cross" decomposition of the Fourier components, 


$$
h_{a b}(t, \vec{x})=\int_{-\infty}^{\infty} \mathrm{d} f \int_{S^{2}} \mathrm{~d}^{2} \Omega_{\hat{k}}\left[h_{+}(f, \hat{k}) e_{a b}^{+}(\hat{k})+h_{\times}(f, \hat{k}) e_{a b}^{\times}(\hat{k})\right] e^{i 2 \pi f(t-\hat{k} \cdot \vec{x} / c)},
$$

we see that

$$
\begin{aligned}
& h_{+}(f, \hat{k})=\sum_{(l m)} \frac{N_{l}}{2}\left[a_{(l m)}^{G}(f) W_{(l m)}(\hat{k})-a_{(l m)}^{C}(f) X_{(l m)}(\hat{k})\right], \\
& h_{\times}(f, \hat{k})=\sum_{(l m)} \frac{N_{l}}{2}\left[a_{(l m)}^{G}(f) X_{(l m)}(\hat{k})+a_{(l m)}^{C}(f) W_{(l m)}(\hat{k})\right],
\end{aligned}
$$

and, conversely,

$$
\begin{aligned}
& a_{(l m)}^{G}(f)=N_{l} \int_{S^{2}} \mathrm{~d}^{2} \Omega_{\hat{k}}\left[h_{+}(f, \hat{k}) W_{(l m)}^{*}(\hat{k})+h_{\times}(f, \hat{k}) X_{(l m)}^{*}(\hat{k})\right], \\
& a_{(l m)}^{C}(f)=N_{l} \int_{S^{2}} \mathrm{~d}^{2} \Omega_{\hat{k}}\left[h_{\times}(f, \hat{k}) W_{(l m)}^{*}(\hat{k})-h_{+}(f, \hat{k}) X_{(l m)}^{*}(\hat{k})\right] .
\end{aligned}
$$

Finally, in terms of spin-weighted spherical harmonics

$$
Y_{(l m) a b}^{G}(\hat{k}) \pm i Y_{(l m) a b}^{C}(\hat{k})=\frac{1}{\sqrt{2}}\left(e_{a b}^{+}(\hat{k}) \pm i e_{a b}^{\times}(\hat{k})\right)_{\mp 2} Y_{(l m)}(\hat{k})
$$

and

$$
\begin{aligned}
h_{+}(f, \hat{k}) \pm i h_{\times}(f, \hat{k}) & =\frac{1}{\sqrt{2}} \sum_{(l m)}\left(a_{(l m)}^{G}(f) \pm i a_{(l m)}^{C}(f)\right)_{ \pm 2} Y_{(l m)}(\hat{k}), \\
\frac{1}{\sqrt{2}}\left(a_{(l m)}^{G}(f) \pm i a_{(l m)}^{C}(f)\right) & =\int \mathrm{d}^{2} \Omega_{\hat{k}}\left(h_{+}(f, \hat{k}) \pm i h_{\times}(f, \hat{k})\right)_{ \pm 2} Y_{(l m)}^{*}(\hat{k}) .
\end{aligned}
$$

These latter expressions for $h_{+}(f, \hat{k}), h_{\times}(f, \hat{k}), a_{(l m)}^{G}(f)$, and $a_{(l m)}^{C}(f)$ are convenient when one can make use of relations derived for the spin-weighted spherical harmonics ${ }_{ \pm 2} Y_{(l m)}(\hat{k})$ (see, e.g., Appendix A).

\section{Statistical properties of the background}

The statistical properties of a Gaussian-stationary background are encoded in the quadratic expectation values $\left\langle h_{A}(f, \hat{k}) h_{A^{\prime}}^{*}\left(f^{\prime}, \hat{k}^{\prime}\right)\right\rangle$ or, equivalently, $\left\langle a_{l m}^{P}(f) a_{l^{\prime} m^{\prime}}^{P^{\prime}}\left(f^{\prime}\right)\right\rangle$, for $A, A^{\prime}=\{+, \times\}$ and $P, P^{\prime}=\{G, C\}$. For a statistically unpolarized and uncorrelated isotropic background

$$
\begin{aligned}
&\left\langle h_{+}(f, \hat{k}) h_{+}^{*}\left(f^{\prime}, \hat{k}^{\prime}\right)\right\rangle=\left\langle h_{\times}(f, \hat{k}) h_{\times}^{*}\left(f^{\prime}, \hat{k}^{\prime}\right)\right\rangle=\frac{1}{2} H(f) \delta^{2}\left(\hat{k}, \hat{k}^{\prime}\right) \delta\left(f-f^{\prime}\right), \\
&\left\langle h_{+}(f, \hat{k}) h_{\times}^{*}\left(f^{\prime}, \hat{k}^{\prime}\right)\right\rangle=\left\langle h_{\times}(f, \hat{k}) h_{+}^{*}\left(f^{\prime}, \hat{k}^{\prime}\right)\right\rangle=0,
\end{aligned}
$$

where $H(f)>0$. The factor of $1 / 2$ has been included so that $H(f)$ is the two-sided gravitational-wave strain power, when summed over both polarizations. Using Eq. (28) and assuming the above expectation values, it follows that

$$
\begin{aligned}
\left\langle a_{(l m)}^{G}(f) a_{\left(l^{\prime} m^{\prime}\right)}^{G *}\left(f^{\prime}\right)\right\rangle= & N_{l} N_{l}^{\prime} \int \mathrm{d}^{2} \Omega_{\hat{k}} \int \mathrm{d}^{2} \Omega_{\hat{k}^{\prime}}\left\langle\left[h_{+}(f, \hat{k}) W_{(l m)}^{*}(\hat{k})+h_{\times}(f, \hat{k}) X_{(l m)}^{*}(\hat{k})\right]\right. \\
& \left.\times\left[h_{+}^{*}\left(f^{\prime}, \hat{k}^{\prime}\right) W_{\left(l^{\prime} m^{\prime}\right)}\left(\hat{k}^{\prime}\right)+h_{\times}^{*}\left(f^{\prime}, \hat{k}^{\prime}\right) X_{\left(l^{\prime} m^{\prime}\right)}\left(\hat{k}^{\prime}\right)\right]\right\rangle \\
& =\frac{N_{l} N_{l^{\prime}}}{2} \int \mathrm{d}^{2} \Omega_{\hat{k}} \int \mathrm{d}^{2} \Omega_{\hat{k}^{\prime}} \delta^{2}\left(\hat{k}, \hat{k}^{\prime}\right)\left[W_{(l m)}^{*}(\hat{k}) W_{\left(l^{\prime} m^{\prime}\right)}\left(\hat{k}^{\prime}\right)+X_{(l m)}^{*}(\hat{k}) X_{\left(l^{\prime} m^{\prime}\right)}\left(\hat{k}^{\prime}\right)\right] H(f) \delta\left(f-f^{\prime}\right) \\
& =\delta_{l l^{\prime}} \delta_{m m^{\prime}} H(f) \delta\left(f-f^{\prime}\right),
\end{aligned}
$$


where the last line follows from the orthogonality relation

$$
\int_{S^{2}} \mathrm{~d}^{2} \Omega_{\hat{k}}\left[W_{(l m)}^{*}(\hat{k}) W_{\left(l^{\prime} m^{\prime}\right)}(\hat{k})+X_{(l m)}^{*}(\hat{k}) X_{\left(l^{\prime} m^{\prime}\right)}(\hat{k})\right]=\frac{2}{N_{l} N_{l}^{\prime}} \delta_{l l^{\prime}} \delta_{m m^{\prime}}
$$

which is a consequence of Eqs. (7) and (8). In a similar way, one can show that

$$
\begin{aligned}
& \left\langle a_{(l m)}^{C}(f) a_{\left(l^{\prime} m^{\prime}\right)}^{C *}\left(f^{\prime}\right)\right\rangle=\delta_{l l^{\prime}} \delta_{m m^{\prime}} H(f) \delta\left(f-f^{\prime}\right), \\
& \left\langle a_{(l m)}^{G}(f) a_{\left(l^{\prime} m^{\prime}\right)}^{C *}\left(f^{\prime}\right)\right\rangle=0=\left\langle a_{(l m)}^{C}(f) a_{\left(l^{\prime} m^{\prime}\right)}^{G *}\left(f^{\prime}\right)\right\rangle,
\end{aligned}
$$

where the zero expectation values follow from

$$
\int_{S^{2}} \mathrm{~d}^{2} \Omega_{\hat{k}}\left[X_{(l m)}^{*}(\hat{k}) W_{\left(l^{\prime} m^{\prime}\right)}(\hat{k})-W_{(l m)}^{*}(\hat{k}) X_{\left(l^{\prime} m^{\prime}\right)}(\hat{k})\right]=0
$$

which is a consequence of Eq. (9). Thus, if we define

$$
\left\langle a_{(l m)}^{P}(f) a_{\left(l^{\prime} m^{\prime}\right)}^{P^{\prime *}}\left(f^{\prime}\right)\right\rangle=\delta_{l l^{\prime}} \delta_{m m^{\prime}} C_{l}^{P P^{\prime}}(f) \delta\left(f-f^{\prime}\right),
$$

where the correlation functions have the form

$$
C_{l}^{P P^{\prime}}(f) \equiv C_{l}^{P P^{\prime}} H(f)
$$

we deduce that an isotropic, unpolarized and uncorrelated background may be described by Eqs. (37) and (38) with

$$
C_{l}^{G G}=C_{l}^{C C} \equiv C_{l}=1, \quad C_{l}^{G C}=0=C_{l}^{C G},
$$

for $l \geq 2$.

\section{Statistically isotropic backgrounds}

Stochastic backgrounds described by expectation values of the form given in Eq. (37) are said to be statistically isotropic. This means that there is no preferred direction on the sky, even though there can be nontrivial angular dependence in the distribution of gravitational-wave power via the $C_{l}^{P P^{\prime}}(f)$. The fact that the quadratic expectation values in Eq. (37) depend only on $l$ and not on $m$ is equivalent to the statement that the angular distribution is independent of the orientation of the reference frame in which it is evaluated. In Sec. IVA, we will extend our analysis to include more general (i.e., statistically anisotropic) backgrounds, allowing expectation values that can also depend on $m$; cf. Eq. (106). In principle, the correlation functions $C_{l}^{P P^{\prime}}(f)$ for a statistically isotropic background are arbitrary, but if we impose additional physicality constraints the forms are restricted, as we shall discuss in Sec. VI A. Requiring the background to be statistically unpolarized imposes the restrictions

$$
\begin{aligned}
& C_{l}^{G G}(f)=C_{l}^{C C}(f) \equiv C_{l}(f), \\
& C_{l}^{G C}(f)=-C_{l}^{C G}(f),
\end{aligned}
$$

which follow from invariance of the expectation values under rotations about a point on the sky. In addition, invariance of the expectation values under a parity transformation $(\hat{k} \rightarrow-\hat{k})$ further requires

$$
C_{l}^{G C}(f)=0=C_{l}^{C G}(f) .
$$

To see that this is indeed the case, recall that under a parity transformation [cf. Eq. (13)],

$$
\begin{aligned}
& a_{(l m)}^{G}(f) \rightarrow(-1)^{l} a_{(l m)}^{G}(f), \\
& a_{(l m)}^{C}(f) \rightarrow(-1)^{l+1} a_{(l m)}^{C}(f),
\end{aligned}
$$

for which

$$
\begin{aligned}
\left\langle a_{(l m)}^{G}(f) a_{\left(l^{\prime} m^{\prime}\right)}^{C *}\left(f^{\prime}\right)\right\rangle & \rightarrow(-1)^{l+l^{\prime}+1}\left\langle a_{(l m)}^{G}(f) a_{\left(l^{\prime} m^{\prime}\right)}^{C *}\left(f^{\prime}\right)\right\rangle \\
& =(-1)^{l+l^{\prime}+1} \delta_{l l^{\prime}} \delta_{m m^{\prime}} C_{l}^{G C}(f) \delta\left(f-f^{\prime}\right) \\
& =-\delta_{l l^{\prime}} \delta_{m m^{\prime}} C_{l}^{G C}(f) \delta\left(f-f^{\prime}\right) \\
& =-\left\langle a_{(l m)}^{G}(f) a_{\left(l^{\prime} m^{\prime}\right)}^{C *}\left(f^{\prime}\right)\right\rangle .
\end{aligned}
$$

Thus, invariance under a parity transformation requires

$$
\left\langle a_{(l m)}^{G}(f) a_{\left(l^{\prime} m^{\prime}\right)}^{C *}\left(f^{\prime}\right)\right\rangle=0
$$

so $C_{l}^{G C}(f)=0$. Similarly, one can show $C_{l}^{C G}(f)=0$. Hence, a statistically isotropic, unpolarized and parityinvariant background is completely characterized by the single correlation function $C_{l}(f) \equiv C_{l}^{G G}(f)=C_{l}^{C C}(f)$. 


\section{E. Detector response functions}

The response of a detector to a passing gravitational wave is given by the convolution of the metric perturbations $h_{a b}(t, \vec{x})$ with the impulse response $R^{a b}(t, \vec{x})$ of the detector,

$$
r(t)=\int_{-\infty}^{\infty} \mathrm{d} \tau \int \mathrm{d}^{3} y R^{a b}(\tau, \vec{y}) h_{a b}(t-\tau, \vec{x}-\vec{y}) .
$$

If we expand the metric perturbations in terms of the plus and cross Fourier modes $h_{A}(f, k)$, where $A=\{+, \times\}$, we can write the response as

$r(t)=\int_{-\infty}^{\infty} \mathrm{d} f \int_{S^{2}} \mathrm{~d}^{2} \Omega_{\hat{k}} \sum_{A} R^{A}(f, \hat{k}) h_{A}(f, \hat{k}) e^{i 2 \pi f t}$,

where

$$
\begin{aligned}
R^{A}(f, \hat{k})= & e^{-i 2 \pi f \hat{k} \cdot \vec{x} / c} e_{a b}^{A}(\hat{k}) \\
& \times \int_{-\infty}^{\infty} \mathrm{d} \tau \int \mathrm{d}^{3} y R^{a b}(\tau, \vec{y}) e^{-i 2 \pi f(\tau-\hat{k} \cdot \vec{y} / c)} .
\end{aligned}
$$

Alternatively, if we expand the metric perturbations in terms of the gradient and curl spherical harmonic modes $a_{(l m)}^{P}(f)$, where $P=\{G, C\}$, we have

$$
r(t)=\int_{-\infty}^{\infty} \mathrm{d} f \sum_{(l m)} \sum_{P} R_{(l m)}^{P}(f) a_{(l m)}^{P}(f) e^{i 2 \pi f t},
$$

where

$$
\begin{aligned}
R_{(l m)}^{P}(f)= & \int_{S^{2}} \mathrm{~d}^{2} \Omega_{\hat{k}} e^{-i 2 \pi f \hat{k} \cdot \vec{x} / c} Y_{(l m) a b}^{P}(\hat{k}) \\
& \times \int_{-\infty}^{\infty} \mathrm{d} \tau \int \mathrm{d}^{3} y R^{a b}(\tau, \vec{y}) e^{-i 2 \pi f(\tau-\hat{k} \cdot \vec{y} / c)} .
\end{aligned}
$$

The detector response functions implicitly depend on the assumptions made about the choice of polarization axes, but we will assume these are consistent with the definitions used in Eqs. (15) and (16) above. Note that the response functions for the two different mode decompositions are related by

$R^{+}(f, \hat{k})=\sum_{(l m)} N_{l}\left[R_{(l m)}^{G}(f) W_{(l m)}^{*}(\hat{k})-R_{(l m)}^{C}(f) X_{(l m)}^{*}(\hat{k})\right]$,
$R^{\times}(f, \hat{k})=\sum_{(l m)} N_{l}\left[R_{(l m)}^{G}(f) X_{(l m)}^{*}(\hat{k})+R_{(l m)}^{C}(f) W_{(l m)}^{*}(\hat{k})\right]$,

and, conversely,

$$
\begin{aligned}
R_{(l m)}^{G}(f)=\frac{N_{l}}{2} \int_{S^{2}} \mathrm{~d}^{2} \Omega_{\hat{k}} & R^{+}(f, \hat{k}) W_{(l m)}(\hat{k}) \\
+ & \left.R^{\times}(f, \hat{k}) X_{(l m)}(\hat{k})\right], \\
R_{(l m)}^{C}(f)=\frac{N_{l}}{2} \int_{S^{2}} \mathrm{~d}^{2} \Omega_{\hat{k}} & R^{\times}(f, \hat{k}) W_{(l m)}(\hat{k}) \\
& \left.-R^{+}(f, \hat{k}) X_{(l m)}(\hat{k})\right],
\end{aligned}
$$

which follow from Eq. (17).

\section{F. Overlap reduction function}

Using Eqs. (37) and (38) for a statistically isotropic background, and assuming $C_{l}^{G G}=C_{l}^{C C} \equiv C_{l}$ and $C_{l}^{G C}=$ $0=C_{l}^{C G}$, the expectation value of the correlation between two detectors, labeled by 1 and 2, can be written as

$$
\left\langle r_{1}(t) r_{2}\left(t^{\prime}\right)\right\rangle=\int_{-\infty}^{\infty} \mathrm{d} f e^{i 2 \pi f\left(t-t^{\prime}\right)} H(f) \Gamma_{12}(f),
$$

where $\Gamma_{12}(f)$ is the overlap reduction function (see, e.g., [45-47]) and is given by

$$
\Gamma_{12}(f)=\sum_{l=2}^{\infty} C_{l} \Gamma_{12, l}(f),
$$

with

$$
\Gamma_{12, l}(f) \equiv \sum_{m=-l}^{l} \sum_{P} R_{1(l m)}^{P}(f) R_{2(l m)}^{P_{*}^{*}}(f),
$$

where $R_{I(I m)}^{P}(f)$ are the gradient and curl response functions for the two detectors, $I=1,2$. In Sec. IVA, we will extend our analysis to compute overlap reduction functions for general anisotropic backgrounds.

\section{APPLICATION TO PULSAR TIMING ARRAYS}

In this section, we apply the above formalism to PTAs, deriving the overlap reduction function for statistically isotropic backgrounds, and showing how one can recover the Hellings and Downs curve. The same approach can also be used to characterize gravitational-wave backgrounds in other frequency bands, relevant to ground-based or spacebased detectors. Although the overlap reduction functions in those cases will be different due to the different detector response functions, they can be calculated in a similar way to the pulsar timing response derived here.

\section{A. Detector response functions}

As a plane gravitational wave transits the Earth-pulsar line of sight, it creates a perturbation in the intervening 
metric, causing a change in the proper separation, which is manifested as a redshift in the pulse frequency [26-29],

$$
z(t, \hat{k}) \equiv \frac{\Delta v(t)}{\nu_{0}}=\frac{1}{2} \frac{u^{a} u^{b}}{1+\hat{k} \cdot \hat{u}} \Delta h_{a b}(t, \hat{k})
$$

where $\hat{k}$ is the direction of propagation of the gravitational wave, $\hat{u}$ is the direction to the pulsar, and $\Delta h_{a b}(t, \hat{k})$ is the difference between the metric perturbation at Earth, $(t, \vec{x})$, and at the pulsar, $\left(t_{p}, \vec{x}_{p}\right)=$ $(t-L / c, \vec{x}+L \hat{u})$,

$$
\begin{aligned}
\Delta h_{a b}(t, \hat{k}) & \equiv \int_{-\infty}^{\infty} \mathrm{d} f h_{a b}(f, \hat{k})\left[e^{i 2 \pi f(t-\hat{k} \cdot \vec{x} / c)}-e^{i 2 \pi f\left(t_{p}-\hat{k} \cdot \vec{x}_{p} / c\right)}\right] \\
& =\int_{-\infty}^{\infty} \mathrm{d} f h_{a b}(f, \hat{k}) e^{i 2 \pi f(t-\hat{k} \cdot \vec{x} / c)}\left[1-e^{-i 2 \pi f L(1+\hat{k} \cdot \hat{u}) / c}\right] .
\end{aligned}
$$

For a gravitational-wave background, which is a superposition of plane waves from all directions on the sky, the pulsar redshift integrated over $\hat{k}$ is given by

$$
z(t)=\int_{-\infty}^{\infty} \mathrm{d} f \int_{S^{2}} \mathrm{~d}^{2} \Omega_{\hat{k}} \frac{1}{2} \frac{u^{a} u^{b}}{1+\hat{k} \cdot \hat{u}} h_{a b}(f, \hat{k}) e^{i 2 \pi f(t-\hat{k} \cdot \vec{x} / c)}\left[1-e^{-i 2 \pi f L(1+\hat{k} \cdot \hat{u}) / c}\right] .
$$

The quantity that is actually observed by a pulsar timing measurement is the timing residual $r(t)$, which is related to the redshift $z(t)$ via

$$
\begin{aligned}
r(t) & \equiv \int_{0}^{t} \mathrm{~d} t^{\prime} z\left(t^{\prime}\right) \\
& =\int_{-\infty}^{\infty} \mathrm{d} f \frac{1}{i 2 \pi f} \int_{S^{2}} \mathrm{~d}^{2} \Omega_{\hat{k}} \frac{1}{2} \frac{u^{a} u^{b}}{1+\hat{k} \cdot \hat{u}} h_{a b}(f, \hat{k}) e^{i 2 \pi f(t-\hat{k} \cdot \vec{x} / c)}\left[1-e^{-i 2 \pi f L(1+\hat{k} \cdot \hat{u}) / c}\right] .
\end{aligned}
$$

If we expand $h_{a b}(f, \hat{k})$ in terms of either $h^{A}(f, \hat{k})$ or $a_{(l m)}^{P}(f)$ [see Eqs. (26) and (14)], and then compare the above expressions with Eqs. (46) and (48), we see that the detector response functions for the timing residuals $r(t)$ are given by

$$
\begin{gathered}
R^{A}(f, \hat{k})=\frac{1}{i 2 \pi f} \frac{1}{2} \frac{u^{a} u^{b}}{1+\hat{k} \cdot \hat{u}} e_{a b}^{A}(\hat{k}) e^{-i 2 \pi f \hat{k} \cdot \vec{x} / c}\left[1-e^{-i 2 \pi f L(1+\hat{k} \cdot \hat{u}) / c}\right], \\
R_{(l m)}^{P}(f)=\frac{1}{i 2 \pi f} \int_{S^{2}} \mathrm{~d}^{2} \Omega_{\hat{k}} \frac{1}{2} \frac{u^{a} u^{b}}{1+\hat{k} \cdot \hat{u}} Y_{(l m) a b}^{P}(\hat{k}) e^{-i 2 \pi f \hat{k} \cdot \vec{x} / c}\left[1-e^{-i 2 \pi f L(1+\hat{k} \cdot \hat{u}) / c}\right] .
\end{gathered}
$$

The detector response functions for the redshift $z(t)$ are the above expressions without the factors of $1 /(i 2 \pi f)$.

In what follows, we will make the approximations

$$
\begin{aligned}
R^{A}(f, \hat{k}) & =(i 2 \pi f)^{-1} F^{A}(\hat{k}), \\
F^{A}(\hat{k}) & \equiv \frac{1}{2} \frac{u^{a} u^{b}}{1+\hat{k} \cdot \hat{u}} e_{a b}^{A}(\hat{k}),
\end{aligned}
$$

and

$$
\begin{aligned}
R_{(l m)}^{P}(f) & =(i 2 \pi f)^{-1} F_{(l m)}^{P}, \\
F_{(l m)}^{P} & \equiv \int_{S^{2}} \mathrm{~d}^{2} \Omega_{\hat{k}} \frac{1}{2} \frac{u^{a} u^{b}}{1+\hat{k} \cdot \hat{u}} Y_{(l m) a b}^{P}(\hat{k}) .
\end{aligned}
$$

This amounts to (i) choosing a reference frame with the origin at the solar-system barycenter (SSB), for which a detector (i.e., a radio telescope on Earth) has $\vec{x} \approx \overrightarrow{0}$, and (ii) omitting the pulsar term, which is proportional to $\exp [-i 2 \pi f L(1+\hat{k} \cdot \hat{u}) / c]$. In the case of an uncorrelated background, the contribution from this term averages to zero in the limit $f L / c \rightarrow \infty$, except for the autocorrelation of each individual pulsar, which this term increases by a factor of 2. The integrand that enters the cross-correlation $\left\langle r_{1}(t) r_{2}\left(t^{\prime}\right)\right\rangle$ for an uncorrelated background contains the factor (see, e.g., [34])

$$
\kappa_{12}(f, \hat{k})=\left[1-e^{-i 2 \pi f L_{1}\left(1+\hat{k} \cdot \hat{u}_{1}\right) / c}\right]\left[1-e^{i 2 \pi f L_{2}\left(1+\hat{k} \cdot \hat{u}_{2}\right) / c}\right] .
$$

For the autocorrelation of data from pulsar 1 with itself, this factor becomes

$$
\left|1-e^{-i 2 \pi f L_{1}\left(1+\hat{k} \cdot \hat{u}_{1}\right) / c}\right|^{2}=2-2 \cos \left[2 \pi f L_{1}\left(1+\hat{k} \cdot \hat{u}_{1}\right) / c\right] .
$$


It is clear that for $f L_{1} / c \gg 1$ the contribution of the oscillatory term to the integral for the overlap reduction function will be suppressed by a factor of a least $1 /\left(f L_{1} / c\right)$ and can hence be ignored. In fact, for an isotropic uncorrelated background, the contribution from the oscillatory term is always small as it is suppressed by a factor of $1 /\left(f L_{1} / c\right)^{2}$. (Details of this calculation are given in Appendix C.) As the angular separation between pulsar pairs increases from zero, the value of the overlap reduction function decreases rapidly to the Earth-term only value. This is a continuous transition; however, for $f L_{1} / c \gg 1$, it is well modeled by a step function at zero angular separation of the pulsar pair. In the following analysis we will consider only interpulsar correlations and can therefore ignore the pulsar term. For a more rigorous investigation of when the pulsar term can be ignored, see, e.g., [48].

\section{B. Antenna beam patterns and pulsar response sky maps}

Figure 1 shows Mollweide projections of the frequencyindependent response functions $F^{+}(\hat{k}), F^{\times}(\hat{k})$ for a pulsar located in direction $(\theta, \phi)=\left(50^{\circ}, 60^{\circ}\right)$, which corresponds to $\left(40^{\circ} \mathrm{N}, 60^{\circ} \mathrm{E}\right)$ in the two plots. Note that the two sky maps are related by a rotation of $45^{\circ}$ around an axis passing through the direction to the pulsar. Plots of $F^{+}(\hat{k}), F^{\times}(\hat{k})$ are sometimes referred to as "antenna beam patterns" in the literature, e.g., [34]. In Fig. 2, we plot the real and imaginary parts of the Fourier transform of the redshift response

$$
\tilde{z}(f)=\int_{S^{2}} \mathrm{~d}^{2} \Omega_{\hat{k}}\left[F^{+}(\hat{k}) h_{+}(f, \hat{k})+F^{\times}(\hat{k}) h_{\times}(f, \hat{k})\right]
$$

to a + -polarized point source $h_{+}(f, \hat{k}) \equiv \delta^{2}\left(\hat{k},-\hat{k}_{0}\right)$, $h_{\times}(f, \hat{k}) \equiv 0$ located in direction $\hat{k}_{0}$ having angular coordinates $\left(\theta_{0}, \phi_{0}\right)=\left(50^{\circ}, 60^{\circ}\right)$. In this pulsar response sky map, the point source is fixed, but the direction to the pulsar is variable, specified by the angles $(\theta, \phi)$. If we had the ability to move pulsars on the sky, this map shows us where the pulsars should ideally be placed to maximize the magnitude of the response (the dark red and dark blue areas). As a simple example, one can show that $\tilde{z}(f)=$ $\frac{1}{2}(1+\cos \theta) \cos (2 \phi)$ for a +-polarized point source located at the North Pole. For a more complicated
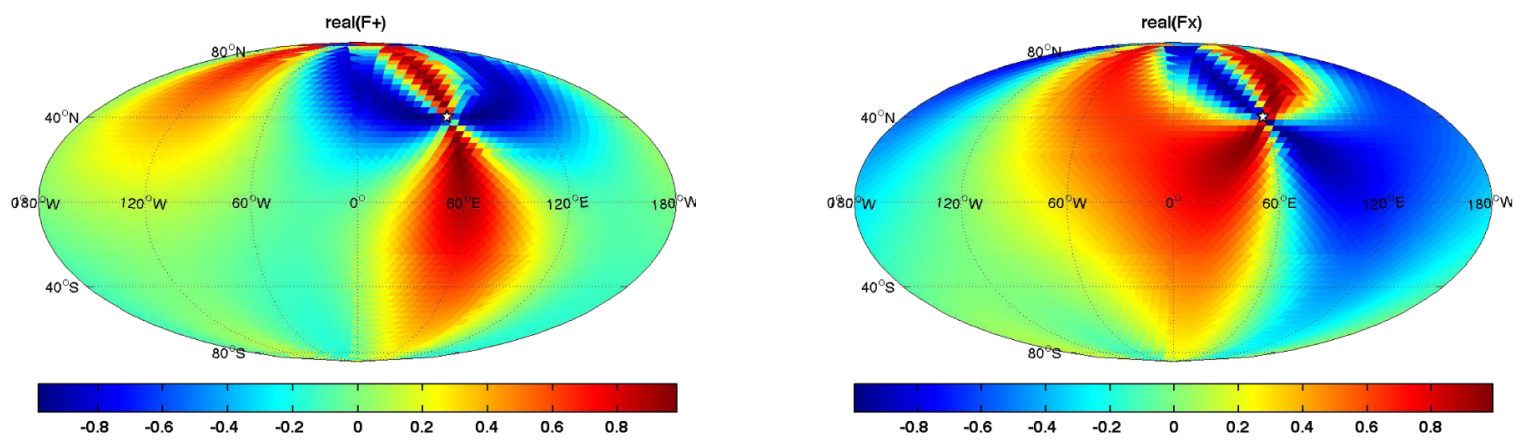

FIG. 1 (color online). Mollweide projections of the frequency-independent response functions $F^{+}(\hat{k}), F^{\times}(\hat{k})$, for a pulsar located in direction $(\theta, \phi)=\left(50^{\circ}, 60^{\circ}\right)$, indicated on the plots by a white star. The imaginary parts of both response functions are identically zero and are not shown above.
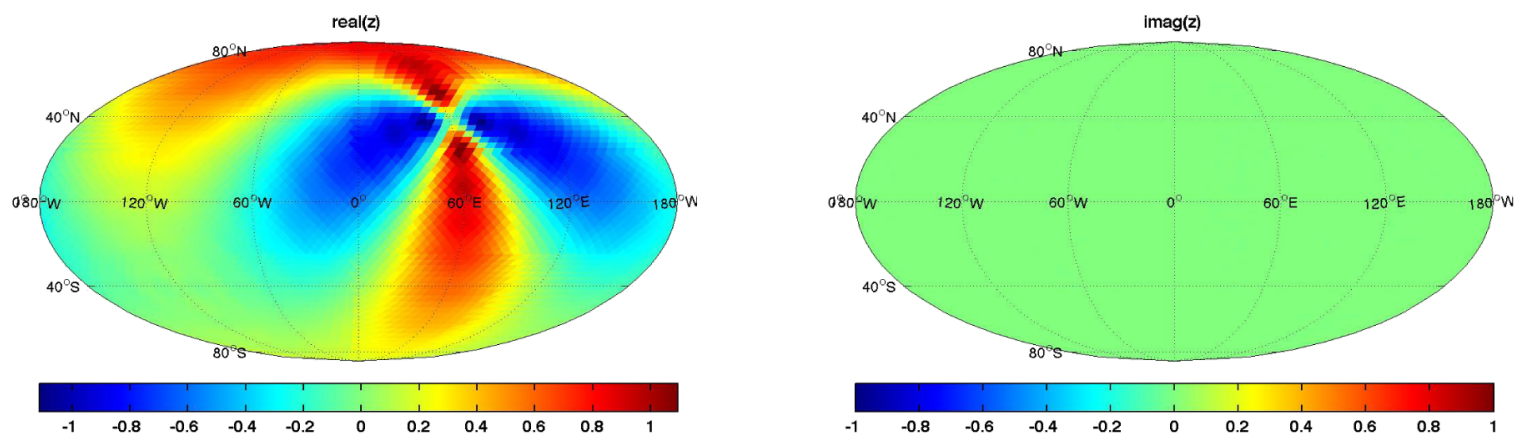

FIG. 2 (color online). Mollweide projections of the real and imaginary parts of the Fourier transform of the redshift response $\tilde{z}(f)$ to a + -polarized point source located in direction $\left(\theta_{0}, \phi_{0}\right)=\left(50^{\circ}, 60^{\circ}\right)$. The sky location of the pulsar is variable. The imaginary part of the response is identically zero, indicated by the solid green color of the second plot. 

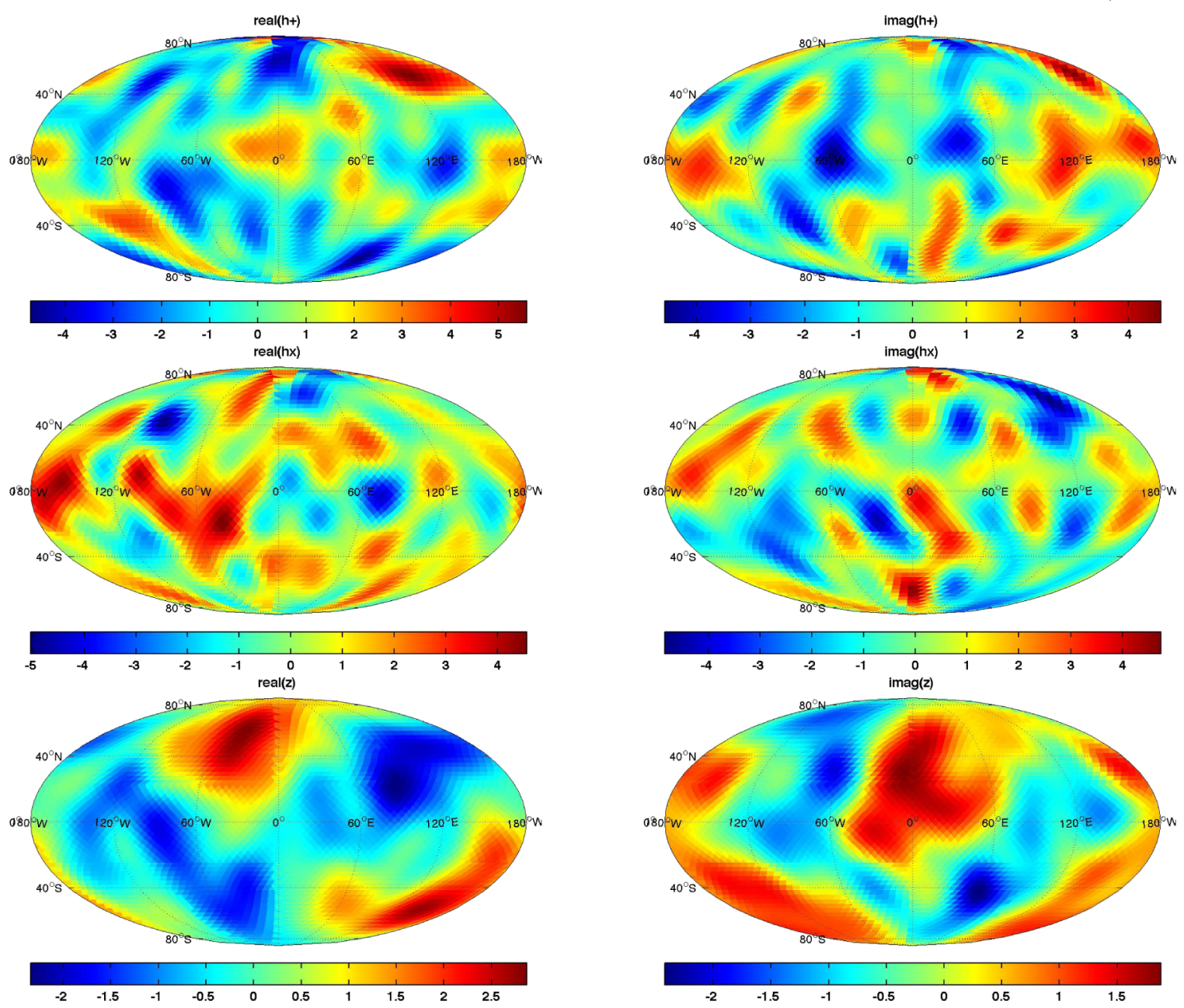

FIG. 3 (color online). Mollweide projections of the real and imaginary parts of $h_{+}(\hat{k}), h_{\times}(\hat{k})$ for a simulated gravitational-wave background (top two rows) and the corresponding pulsar response map (bottom row).

gravitational-wave background, the pulsar response map is shown in Fig. 3.

Note that the Fourier transform of the timing residual response $\tilde{r}(f)$ is related to the Fourier transform of the redshift response $\tilde{z}(f)$ by the frequency-dependent scaling $\tilde{r}(f)=\tilde{z}(f) /(i 2 \pi f)$, which follows from Eq. (58). So it is a simple matter to go back and forth between these two types of response.

\section{Overlap reduction function}

Consider two pulsars, labeled by 1 and 2 , and let $\hat{u}_{1}, \hat{u}_{2}$ be unit vectors pointing from Earth to each pulsar. To calculate the expected value of the correlation between the signals from these two pulsars due to a statistically isotropic gravitational-wave background, we need to evaluate the integrals (51) that appear in Eq. (54) for the components of the overlap reduction function. This can be done in any reference frame. Here we follow the standard approach of $[34,35]$, and work in the so-called "computational" frame in which pulsar 1 is located on the $z$ axis and pulsar 2 is located in the $x z$ plane, making an angle $\zeta$ with respect to the $z$ axis,

$$
\begin{aligned}
& \hat{u}_{1}=(0,0,1), \\
& \hat{u}_{2}=(\sin \zeta, 0, \cos \zeta) .
\end{aligned}
$$

We will also assume that the detector locations are at the origin (the SSB),

$$
\vec{x}_{1} \approx \vec{x}_{2} \approx \overrightarrow{0}
$$

Furthermore, we will do the calculation in terms of the traditional + and $\times$ detector response functions, making the approximation $R_{I}^{A}(f, \hat{k})=(i 2 \pi f)^{-1} F_{I}^{A}(\hat{k}) \quad($ for $I=1,2)$ discussed above. (In Sec. III D, we will give an alternative derivation of the overlap reduction function, doing the calculation in different reference frames for each pulsar, and working more directly with the gradient and curl response functions.) 
In this computational frame, it is easy to show that

$$
\begin{aligned}
& F_{1}^{+}(\hat{k})=\frac{1}{2} \frac{u_{1}^{a} u_{1}^{b}}{1+\hat{k} \cdot \hat{u}_{1}} e_{a b}^{+}(\hat{k})=\frac{1}{2}(1-\cos \theta), \\
& F_{1}^{\times}(\hat{k})=\frac{1}{2} \frac{u_{1}^{a} u_{1}^{b}}{1+\hat{k} \cdot \hat{u}_{1}} e_{a b}^{\times}(\hat{k})=0 .
\end{aligned}
$$

Since $W_{(l m)}(\hat{k})$ and $X_{(l m)}(\hat{k})$ are both proportional to $e^{i m \phi}$ and $F_{1}^{+}(\hat{k})$ is independent of $\phi$, the integral over $\phi$ gives zero for all $m \neq 0$. We note also that $X_{(10)}=0$ for all $l$, which follows from Eq. (19). Thus, Eq. (54) simplifies to

$$
\Gamma_{12, l}(f)=\frac{1}{(2 \pi f)^{2}} \frac{\left(N_{l}\right)^{2}}{4}\left[\int \mathrm{d}^{2} \Omega_{\hat{k}} F_{1}^{+}(\hat{k}) W_{(l 0)}(\hat{k})\right]\left[\int \mathrm{d}^{2} \Omega_{\hat{k}^{\prime}} F_{2}^{+*}\left(\hat{k}^{\prime}\right) W_{(l 0)}^{*}\left(\hat{k}^{\prime}\right)\right],
$$

where the factor of $(2 \pi f)^{-2}$ comes from the frequency-dependent factors of $(i 2 \pi f)^{-1}$ in Eqs. (61) and (62). Note that the frequency dependence in Eq. (69) is usually absorbed into $H(f)$ in Eq. (52); see, e.g., [35]. We will denote the frequencyindependent part of the overlap reduction functions with an overbar, e.g., $\bar{\Gamma}_{12}$ and $\bar{\Gamma}_{12, l}$.

We will consider the integral over $\hat{k}$ first, making use of Eq. (18) written in terms of $x=\cos \theta$,

$$
\int \mathrm{d}^{2} \Omega_{\hat{k}} F_{1}^{+}(\hat{k}) W_{(l 0)}(\hat{k})=\sqrt{\frac{(2 l+1) \pi}{4}} \int_{-1}^{1} \mathrm{~d} x(1-x)\left(2\left(1-x^{2}\right) \frac{\mathrm{d}^{2}}{\mathrm{~d} x^{2}}-2 x \frac{\mathrm{d}}{\mathrm{d} x}+l(l+1)\right) P_{l}(x),
$$

where $P_{l}(x)$ is a Legendre polynomial. This last expression in brackets can be simplified further, noting that

$$
\left(2\left(1-x^{2}\right) \frac{\mathrm{d}^{2}}{\mathrm{~d} x^{2}}-2 x \frac{\mathrm{d}}{\mathrm{d} x}+l(l+1)\right) P_{l}(x)=\left(1-x^{2}\right) \frac{\mathrm{d}^{2}}{\mathrm{~d} x^{2}} P_{l}(x) .
$$

Thus,

$$
\int \mathrm{d}^{2} \Omega_{\hat{k}} F_{1}^{+}(\hat{k}) W_{(l 0)}(\hat{k})=\sqrt{\frac{(2 l+1) \pi}{4}} \int_{-1}^{1} \mathrm{~d} x(1-x)\left(1-x^{2}\right) \frac{\mathrm{d}^{2}}{\mathrm{~d} x^{2}} P_{l}(x) .
$$

If we now integrate by parts twice, and use the orthogonality of the Legendre polynomials, Eq. (B6) (noting that $l \geq 2$ for this tensor harmonic basis), we find that only the second boundary term contributes, yielding

$$
\int \mathrm{d}^{2} \Omega_{\hat{k}} F_{1}^{+}(\hat{k}) W_{(l 0)}(\hat{k})=2 \sqrt{(2 l+1) \pi}(-1)^{l} .
$$

To evaluate the integral over $\hat{k}^{\prime}$, we note that

$$
\begin{aligned}
F_{2}^{+*}\left(\hat{k}^{\prime}\right) & =\frac{1}{2} \frac{u_{2}^{a} u_{2}^{b}}{1+\hat{k}^{\prime} \cdot \hat{u}_{2}} e_{a b}^{+}\left(\hat{k}^{\prime}\right) \\
& =\frac{1}{2} \frac{\left(\sin \zeta \cos \theta^{\prime} \cos \phi^{\prime}-\cos \zeta \sin \theta^{\prime}\right)^{2}-\left(-\sin \zeta \sin \phi^{\prime}\right)^{2}}{1+\cos \zeta \cos \theta^{\prime}+\sin \zeta \sin \theta^{\prime} \cos \phi^{\prime}} \\
& =\frac{1}{2}\left[\left(1-\cos \zeta \cos \theta^{\prime}-\sin \zeta \sin \theta^{\prime} \cos \phi^{\prime}\right)-\frac{2 \sin ^{2} \zeta \sin ^{2} \phi^{\prime}}{1+\cos \zeta \cos \theta^{\prime}+\sin \zeta \sin \theta^{\prime} \cos \phi^{\prime}}\right]
\end{aligned}
$$

[We do not need to calculate $F_{2}^{\times}\left(\hat{k}^{\prime}\right)$, since it does not enter the expression for the overlap reduction function in our chosen reference frame.] The integral can be written following [34] as a sum of two parts,

$$
\int \mathrm{d}^{2} \Omega_{\hat{k}^{\prime}} F_{2}^{+*}\left(\hat{k}^{\prime}\right) W_{(l 0)}^{*}\left(\hat{k}^{\prime}\right)=Q_{l}+R_{l}
$$

where

$$
Q_{l}=\sqrt{\frac{(2 l+1) \pi}{4}} \int_{-1}^{1} \mathrm{~d} x(1-x \cos \zeta)\left(1-x^{2}\right) \frac{\mathrm{d}^{2}}{\mathrm{~d} x^{2}} P_{l}(x),
$$




$$
R_{l}=-\sqrt{\frac{(2 l+1)}{4 \pi}} \sin ^{2} \zeta \int_{-1}^{1} \mathrm{~d} x\left(1-x^{2}\right) \frac{\mathrm{d}^{2}}{\mathrm{~d} x^{2}} P_{l}(x) \int_{0}^{2 \pi} \mathrm{d} \phi^{\prime} \frac{\sin ^{2} \phi^{\prime}}{1+x \cos \zeta+\sqrt{1-x^{2}} \sin \zeta \cos \phi^{\prime}}
$$

The $Q_{l}$ integral is similar in form to the integral over $\hat{k}$ given above and can be evaluated in the same way,

$$
Q_{l}=\sqrt{(2 l+1) \pi}\left[(1-\cos \zeta)+(1+\cos \zeta)(-1)^{l}\right] .
$$

For $R_{l}$, the integral over $\phi^{\prime}$ is familiar from the standard computation of the Hellings and Downs curve. $R_{l}$ can be reduced to the form

$$
\begin{aligned}
R_{l} & =-\sqrt{(2 l+1) \pi}\left[\int_{-1}^{-\cos \zeta} \mathrm{d} x \frac{(1+\cos \zeta)}{(1-x)}\left(1-x^{2}\right) \frac{\mathrm{d}^{2}}{\mathrm{~d} x^{2}} P_{l}(x)+\int_{-\cos \zeta}^{1} \mathrm{~d} x \frac{(1-\cos \zeta)}{(1+x)}\left(1-x^{2}\right) \frac{\mathrm{d}^{2}}{\mathrm{~d} x^{2}} P_{l}(x)\right] \\
& =-\sqrt{(2 l+1) \pi}\left[(1+\cos \zeta) \int_{-1}^{-\cos \zeta} \mathrm{d} x(1+x) \frac{\mathrm{d}^{2}}{\mathrm{~d} x^{2}} P_{l}(x)+(1-\cos \zeta) \int_{-1}^{-\cos \zeta} \mathrm{d} x(1-x) \frac{\mathrm{d}^{2}}{\mathrm{~d} x^{2}} P_{l}(x)\right] .
\end{aligned}
$$

Integrating each term by parts we obtain

$$
R_{l}=-\sqrt{(2 l+1) \pi}\left[(1-\cos \zeta)+(1+\cos \zeta)(-1)^{l}-2(-1)^{l} P_{l}(\cos \zeta)\right] .
$$

Thus, the $\hat{k}^{\prime}$ integral is simply

$$
\int \mathrm{d}^{2} \Omega_{\hat{k}^{\prime}} F_{2}^{+*}\left(\hat{k}^{\prime}\right) W_{(l 0)}^{*}\left(\hat{k}^{\prime}\right)=2 \sqrt{(2 l+1) \pi}(-1)^{l} P_{l}(\cos \zeta) .
$$

Putting the above results together, we obtain

$$
\Gamma_{12, l}(f)=\frac{1}{(2 \pi f)^{2}}\left(N_{l}\right)^{2}(2 l+1) \pi P_{l}(\cos \zeta) .
$$

Thus, for a statistically isotropic background, the overlap reduction functions for the individual $l$ modes are proportional to Legendre polynomials. The full overlap reduction function, assuming $C_{l}^{G G}=C_{l}^{C C} \equiv C_{l}$ and $C_{l}^{G C}=0=C_{l}^{C G}$, is given by the sum

$$
\Gamma_{12}(f)=\frac{1}{(2 \pi f)^{2}} \sum_{l=2}^{\infty} C_{l}\left(N_{l}\right)^{2}(2 l+1) \pi P_{l}(\cos \zeta) .
$$

\section{Alternative derivation of the overlap reduction function}

The above result for $\Gamma_{12, l}(f)$ is surprisingly simple, considering the somewhat involved calculation needed to derive it. This raises the question as to whether there is an alternative approach that would lead more directly to the final result. The answer to this question is yes, and it is based on the observation that Eq. (54) for $\Gamma_{12, l}(f)$ involves a sum of products of two integrals, each of the form given by Eq. (60). This means that the integrals for pulsars 1 and 2 need not be evaluated in the same reference frame as we did in the previous section, but can be evaluated in different reference frames, appropriately chosen to simplify the calculation of the integral for each pulsar separately. In particular, we can rotate coordinates so that, for each pulsar, $\hat{u}_{I}$ is directed along the transformed $z$ axis.

To be more explicit, consider a particular pulsar $I$ located in direction $\hat{u}_{I}$. In the "cosmic" reference frame, where the angular dependence of the gravitational-wave background is to be described, let the angular coordinates of the pulsar be $\left(\zeta_{I}, \chi_{I}\right)$, so that

$$
u_{I}^{a}=\left(\sin \zeta_{I} \cos \chi_{I}, \sin \zeta_{I} \sin \chi_{I}, \cos \zeta_{I}\right)
$$

A rotation

$$
\mathbf{R}(\alpha, \beta, \gamma)=\mathbf{R}_{z}(\gamma) \mathbf{R}_{y}(\beta) \mathbf{R}_{z}(\alpha)=\left(\begin{array}{ccc}
\cos \gamma & \sin \gamma & 0 \\
-\sin \gamma & \cos \gamma & 0 \\
0 & 0 & 1
\end{array}\right)\left(\begin{array}{ccc}
\cos \beta & 0 & -\sin \beta \\
0 & 1 & 0 \\
\sin \beta & 0 & \cos \gamma
\end{array}\right)\left(\begin{array}{ccc}
\cos \alpha & \sin \alpha & 0 \\
-\sin \alpha & \cos \alpha & 0 \\
0 & 0 & 1
\end{array}\right)
$$


defined by the Euler angles

$$
(\alpha, \beta, \gamma)=\left(\chi_{I}, \zeta_{I}, 0\right)
$$

will rotate the cosmic frame (with coordinates $x^{a}$ ) to the computational frame for pulsar $I$ [with coordinates $\left.x_{I}^{\bar{a}}=\mathbf{R}\left(\chi_{I}, \zeta_{I}, 0\right)^{\bar{a}}{ }_{a} x^{a}\right]$, so that

$$
u_{I}^{\bar{a}}=(0,0,1) .
$$

To evaluate the response functions in the computational frame, we need to know how the integrands transform under a rotation. Ignoring the pulsar term and making the approximation $\vec{x}_{I} \approx \overrightarrow{0}$, these integrals simplify to

$$
\begin{aligned}
R_{I(l m)}^{P}(f) & =(i 2 \pi f)^{-1} F_{I(l m)}^{P}, \\
F_{I(l m)}^{P} & \equiv \int_{S^{2}} \mathrm{~d}^{2} \Omega_{\hat{k}} \frac{1}{2} \frac{u_{I}^{a} u_{I}^{b}}{1+\hat{k} \cdot \hat{u}_{I}} Y_{(l m) a b}^{P}(\hat{k})
\end{aligned}
$$

as mentioned in the text following Eqs. (61) and (62). The frequency-dependent factor $(i 2 \pi f)^{-1}$ is a scalar with respect to rotations, so we need only determine the transformation properties of $F_{I(l m)}^{P}$. Since the gradient and curl spherical harmonics transform like tensors with respect to the $a b$ indices and like ordinary spherical harmonics with respect to the $l m$ indices [40], we have

$$
\begin{aligned}
& Y_{(l m) a b}^{P}(\theta, \phi)=\sum_{m^{\prime}=-l}^{l}\left[D_{m m^{\prime}}^{l}\left(\chi_{I}, \zeta_{I}, 0\right)\right]^{*} \\
& \quad \times Y_{\left(l m^{\prime}\right) \bar{a} \bar{b}}^{P}\left(\bar{\theta}_{I}, \bar{\phi}_{I}\right) \mathbf{R}\left(\chi_{I}, \zeta_{I}, 0\right)^{\bar{a}}{ }_{a} \mathbf{R}\left(\chi_{I}, \zeta_{I}, 0\right)^{\bar{b}}{ }_{b} .
\end{aligned}
$$

Here $D_{m^{\prime} m}^{l}\left(\chi_{I}, \zeta_{I}, 0\right)$ is the Wigner-D matrix associated with the rotation $\mathbf{R}\left(\chi_{I}, \zeta_{I}, 0\right)$, and $\left(\bar{\theta}_{I}, \bar{\phi}_{I}\right)$ are the angular coordinates of the direction $\hat{k}$ in the computational frame for pulsar $I$. Thus, for the gradient response

$$
\begin{aligned}
F_{I(l m)}^{G} & =\int_{0}^{2 \pi} \mathrm{d} \phi \int_{-1}^{1} \mathrm{~d} \cos \theta \frac{1}{2} \frac{u_{I}^{a} u_{I}^{b}}{1+\hat{k} \cdot \hat{u}_{I}} Y_{(l m) a b}^{G}(\theta, \phi) \\
& =\sum_{m^{\prime}=-l}^{l}\left[D^{l}{ }_{m m^{\prime}}\left(\chi_{I}, \zeta_{I}, 0\right)\right]^{*} \int_{0}^{2 \pi} \mathrm{d} \bar{\phi}_{I} \int_{-1}^{1} \mathrm{~d} \cos \bar{\theta}_{I} \frac{1}{2} \frac{u_{I}^{\bar{a}} u_{I}^{\bar{b}}}{1+\hat{k} \cdot \hat{u}_{I}} Y_{\left(l m^{\prime}\right) \bar{a} \bar{b}}^{G}\left(\bar{\theta}_{I}, \bar{\phi}_{I}\right) \\
& =\sum_{m^{\prime}=-l}^{l}\left[D^{l}{ }_{m m^{\prime}}\left(\chi_{I}, \zeta_{I}, 0\right)\right]^{*} \int_{0}^{2 \pi} \mathrm{d} \bar{\phi}_{I} \int_{-1}^{1} \mathrm{~d} \cos \bar{\theta}_{I} \frac{N_{l}}{2}\left[F_{I}^{+}(\bar{\theta}, \bar{\phi}) W_{\left(l m^{\prime}\right)}\left(\bar{\theta}_{I}, \bar{\phi}_{I}\right)+F_{I}^{\times}\left(\bar{\theta}_{I}, \bar{\phi}_{I}\right) X_{\left(l m^{\prime}\right)}\left(\bar{\theta}_{I}, \bar{\phi}_{I}\right)\right],
\end{aligned}
$$

where we used Eq. (51) with the $R$ 's replaced by $F$ 's to get the last line. Since in the computational frame

$$
F_{I}^{+}\left(\bar{\theta}_{I}, \bar{\phi}_{I}\right)=\frac{1}{2}\left(1-\cos \bar{\theta}_{I}\right), \quad F_{I}^{\times}\left(\bar{\theta}_{I}, \bar{\phi}_{I}\right)=0,
$$

we can ignore the $F_{I}^{\times}\left(\bar{\theta}_{I}, \bar{\phi}_{I}\right)$ term. In addition, because $F_{I}^{+}\left(\bar{\theta}_{I}, \bar{\phi}_{I}\right)$ is independent of $\bar{\phi}_{I}$, we only get a contribution from $m^{\prime}=0$. Thus,

$$
\begin{aligned}
F_{I(l m)}^{G} & =\left[D_{m 0}^{l}\left(\chi_{I}, \zeta_{I}, 0\right)\right]^{*} \int_{0}^{2 \pi} \mathrm{d} \bar{\phi}_{I} \int_{-1}^{1} \mathrm{~d} \cos \bar{\theta}_{I} \frac{N_{l}}{2} F_{I}^{+}\left(\bar{\theta}_{I}, \bar{\phi}_{I}\right) W_{(l 0)}\left(\bar{\theta}_{I}, \bar{\phi}_{I}\right) \\
& =\left[D^{l}{ }_{m 0}\left(\chi_{I}, \zeta_{I}, 0\right)\right]^{*} N_{l} \sqrt{(2 l+1) \pi}(-1)^{l} \\
& =2 \pi(-1)^{l} N_{l} Y_{(l m)}\left(\zeta_{I}, \chi_{I}\right),
\end{aligned}
$$

where we used Eq. (73) for the $W_{(10)}\left(\bar{\theta}_{I}, \bar{\phi}_{I}\right)$ integration and Eq. (A6) for the Wigner-D matrix.

Proceeding in exactly the same manner for the curl response, we find

$$
\begin{aligned}
F_{I(l m)}^{C} & =\int_{0}^{2 \pi} \mathrm{d} \phi \int_{-1}^{1} \mathrm{~d} \cos \theta \frac{1}{2} \frac{u_{I}^{a} u_{I}^{b}}{1+\hat{k} \cdot \hat{u}_{I}} Y_{(l m) a b}^{C}(\theta, \phi) \\
& =\sum_{m^{\prime}=-l}^{l}\left[D^{l}{ }_{m m^{\prime}}\left(\chi_{I}, \zeta_{I}, 0\right)\right]^{*} \int_{0}^{2 \pi} \mathrm{d} \bar{\phi}_{I} \int_{-1}^{1} \mathrm{~d} \cos \bar{\theta}_{I} \frac{1}{2} \frac{u_{I}^{\bar{a}} u_{I}^{\bar{b}}}{1+\hat{k} \cdot \hat{u}_{I}} Y_{\left(l m^{\prime}\right) \bar{a} \bar{b}}^{C}\left(\bar{\theta}_{I}, \bar{\phi}_{I}\right) \\
& =\sum_{m^{\prime}=-l}^{l}\left[D^{l}{ }_{m m^{\prime}}\left(\chi_{I}, \zeta_{I}, 0\right)\right]^{*} \int_{0}^{2 \pi} \mathrm{d} \bar{\phi}_{I} \int_{-1}^{1} \mathrm{~d} \cos \bar{\theta}_{I} \frac{N_{l}}{2}\left[F_{I}^{\times}\left(\bar{\theta}_{I}, \bar{\phi}_{I}\right) W_{\left(l m^{\prime}\right)}\left(\bar{\theta}_{I}, \bar{\phi}_{I}\right)-F_{I}^{+}\left(\bar{\theta}_{I}, \bar{\phi}_{I}\right) X_{\left(l m^{\prime}\right)}\left(\bar{\theta}_{I}, \bar{\phi}_{I}\right)\right] \\
& =-\left[D^{l}{ }_{m 0}\left(\chi_{I}, \zeta_{I}, 0\right)\right]^{*} \int_{0}^{2 \pi} \mathrm{d} \bar{\phi}_{I} \int_{-1}^{1} \mathrm{~d} \cos \bar{\theta}_{I} \frac{N_{l}}{2} F_{I}^{+}\left(\bar{\theta}_{I}, \bar{\phi}_{I}\right) X_{(l 0)}\left(\bar{\theta}_{I}, \bar{\phi}_{I}\right) \\
& =0,
\end{aligned}
$$


where the last equality follows from $X_{l 0}\left(\bar{\theta}_{I}, \bar{\phi}_{I}\right)=0$ by Eq. (19). The significance of this result will be discussed in detail in Sec. IVA.

Returning now to Eq. (54), and including the factors of $(i 2 \pi f)^{-1}$, we have

$$
\begin{aligned}
\Gamma_{12, l}(f) & =\sum_{m=-l}^{l} \sum_{P} R_{1(l m)}^{P}(f) R_{2(l m)}^{P *}(f) \\
& =\frac{1}{(2 \pi f)^{2}} 4 \pi^{2}\left(N_{l}\right)^{2} \sum_{m=-l}^{l} Y_{(l m)}\left(\zeta_{1}, \chi_{1}\right) Y_{(l m)}^{*}\left(\zeta_{2}, \chi_{2}\right) \\
& =\frac{1}{(2 \pi f)^{2}}\left(N_{l}\right)^{2}(2 l+1) \pi P_{l}\left(\hat{u}_{1} \cdot \hat{u}_{2}\right),
\end{aligned}
$$

where we used the addition theorem for (ordinary) spherical harmonics, Eq. (A12), to eliminate the summation over $m$. Note that this reproduces the result from the previous subsection, Eq. (82), with $\cos \zeta=\hat{u}_{1} \cdot \hat{u}_{2}$, where $\zeta$ is the angle between the directions to the two pulsars.

\section{E. Recovery of the Hellings and Downs curve}

For an isotropic, unpolarized and uncorrelated gravitational-wave background, we expect to recover the Hellings and Downs curve from the frequency-independent part of the overlap reduction function

$$
\bar{\Gamma}_{12} \equiv \sum_{l=2}^{\infty} C_{l}\left(N_{l}\right)^{2}(2 l+1) \pi P_{l}\left(\hat{u}_{1} \cdot \hat{u}_{2}\right)
$$

For such a background, we saw in Sec. II C that

$$
C_{l}^{G G}=C_{l}^{C C}=C_{l}=1, \quad C_{l}^{G C}=0=C_{l}^{C G},
$$

for $l \geq 2$. To show that these are indeed the coefficients that recover the Hellings and Downs curve, we decompose the Hellings and Downs curve as a superposition of Legendre polynomials

$$
\begin{array}{r}
\frac{1}{2}\left(1+\frac{3}{2}(1-\cos \zeta)\left[\ln \left(\frac{1-\cos \zeta}{2}\right)-\frac{1}{6}\right]\right) \\
=\sum_{l=0}^{\infty} a_{l} P_{l}(\cos \zeta)
\end{array}
$$

The coefficients are given in the usual way

$$
a_{l}=\frac{2 l+1}{2} \int_{-1}^{1} \mathrm{~d} x \frac{1}{2}\left(1+\frac{3}{2}(1-x)\left[\ln \left(\frac{1-x}{2}\right)-\frac{1}{6}\right]\right) P_{l}(x),
$$

which follows from

$$
\int_{-1}^{1} \mathrm{~d} x P_{l}(x) P_{l^{\prime}}(x)=\frac{2}{2 l+1} \delta_{l l^{\prime}}
$$

Considering $a_{0}$ and $a_{1}$ first,

$$
\begin{aligned}
4 a_{0} & =\int_{-1}^{1} \mathrm{~d} x\left(1+\frac{3}{2}(1-x)\left[\ln \left(\frac{1-x}{2}\right)-\frac{1}{6}\right]\right) \\
& =\left[x-\frac{3}{4}(1-x)^{2} \ln \left(\frac{1-x}{2}\right)+\frac{(1-x)^{2}}{2}\right]_{-1}^{1} \\
& =1-(-1+2) \\
& =0
\end{aligned}
$$

and

$$
\begin{aligned}
\frac{4}{3} a_{1}= & \int_{-1}^{1} \mathrm{~d} x\left(x+\frac{3}{2} x(1-x)\left[\ln \left(\frac{1-x}{2}\right)-\frac{1}{6}\right]\right) \\
= & {\left[\frac{3}{8} x^{2}+\left(\frac{1}{2}(1-x)^{3}-\frac{3}{4}(1-x)^{2}\right) \ln \left(\frac{1-x}{2}\right)\right.} \\
& \left.+\frac{3}{8}(1-x)^{2}-\frac{(1-x)^{3}}{6}+\frac{x^{3}}{12}\right]_{-1}^{1} \\
= & \left(\frac{3}{8}+\frac{1}{12}\right)-\left(\frac{3}{8}+\frac{3}{2}-\frac{8}{6}-\frac{1}{12}\right) \\
= & 0 .
\end{aligned}
$$

The vanishing of these coefficients is to be expected, as the expansion in Eqs. (10) and (53) starts at $l=2$.

For $l \geq 2$, since $1-(1-x) / 4=3 P_{0}(x) / 4+P_{1}(x) / 4$, orthogonality of the Legendre polynomials ensures

$$
\begin{aligned}
\frac{4}{2 l+1} a_{l} & =\int_{-1}^{1} \mathrm{~d} x \frac{3}{2}(1-x) \ln \left(\frac{1-x}{2}\right) P_{l}(x) \\
& =6 \int_{0}^{1} \mathrm{~d} y y \ln (y) P_{l}(1-2 y),
\end{aligned}
$$

where we have made the substitution $y=(1-x) / 2$. The shifted Legendre polynomials $\tilde{P}_{l}(y) \equiv P_{l}(1-2 y)$ are given by the formula

$$
\tilde{P}_{l}(y)=\frac{1}{l !} \frac{\mathrm{d}^{l}}{\mathrm{~d} y^{l}}\left[\left(y-y^{2}\right)^{l}\right]
$$

and therefore we obtain 


$$
\begin{aligned}
\frac{4}{2 l+1} a_{l} & =\frac{6}{l !} \int_{0}^{1} \mathrm{~d} y y \ln (y) \frac{\mathrm{d}^{l}}{\mathrm{~d} y^{l}}\left[\left(y-y^{2}\right)^{l}\right] \\
& =\frac{6}{l !}\left[y \ln (y) \frac{\mathrm{d}^{l-1}}{\mathrm{~d} y^{l-1}}\left[\left(y-y^{2}\right)^{l}\right]\right]_{0}^{1}-\frac{6}{l !} \int_{0}^{1} \mathrm{~d} y(1+\ln (y)) \frac{\mathrm{d}^{l-1}}{\mathrm{~d} y^{l-1}}\left[\left(y-y^{2}\right)^{l}\right] \\
& =-\frac{6}{l !}\left[(1+\ln (y)) \frac{\mathrm{d}^{l-2}}{\mathrm{~d} y^{l-2}}\left[\left(y-y^{2}\right)^{l}\right]\right]_{0}^{1}+\frac{6}{l !} \int_{0}^{1} \mathrm{~d} y \frac{1}{y} \frac{\mathrm{d}^{l-2}}{\mathrm{~d} y^{l-2}}\left[\left(y-y^{2}\right)^{l}\right] \\
& =\frac{6}{l !} \int_{0}^{1} \mathrm{~d} y \frac{1}{y^{2}} \frac{\mathrm{d}^{l-3}}{\mathrm{~d} y^{l-3}}\left[\left(y-y^{2}\right)^{l}\right]=\cdots=\frac{6(l-2) !}{l !} \int_{0}^{1} \mathrm{~d} y \frac{\left(y-y^{2}\right)^{l}}{y^{l-1}}=\frac{6(l-2) !}{l !} \int_{0}^{1} \mathrm{~d} y y(1-y)^{l} \\
& =\frac{6(l-2) !}{l !} \int_{0}^{1} \mathrm{~d} y(1-y) y^{l}=\frac{6(l-2) !}{l !}\left[\frac{y^{l+1}}{l+1}-\frac{y^{l+2}}{l+2}\right]_{0}^{1}=\frac{6(l-2) !}{(l+2) !}=3 N_{l}^{2},
\end{aligned}
$$

where we have used the fact that, for $k<n$, $\mathrm{d}^{k} / \mathrm{d} y^{k}\left[\left(y-y^{2}\right)^{n}\right]=0$ at $y=0,1$. Thus,

$$
a_{l}=\frac{3}{4}\left(N_{l}\right)^{2}(2 l+1) \text {. }
$$

Comparing these coefficients with the $C_{l}$ given in Eq. (95) for two pulsars separated by the angle $\zeta$, we see that the $l$ dependence of the $a_{l}$ agrees with $C_{l}=\operatorname{const}(=3 / 4 \pi)$ for $l \geq 2$, precisely as found above. We note that, since the correlation function for an isotropic background must depend only on the pulsar separation, we would expect to be able to expand it as a sum of Legendre polynomials. This property was exploited in a search for isotropic gravitationalwave backgrounds with non-general relativistic polarizations in [31], where a Legendre polynomial expansion up to $l=5$ was used as a fit to numerically computed correlation curves for longitudinal polarization modes.

We now explore the utility of this decomposition by generating and analyzing a set of pulsar TOAs. The data set was constructed using the GWbkgrd plugin within the pulsar-timing software package TEMPO2 [49-51], which injects an isotropic, unpolarized and uncorrelated gravitational-wave background into a set of specified pulsar observations. We employ an array of 10 pulsars spread uniformly over the sky and observed fortnightly for 5 years. The injected background power spectrum is flat, creating a correlated white-noise influence in pulsars separated across the sky.

Using a Bayesian time-domain likelihood formalism [52,53], we test models of the overlap reduction function which truncate the expansion in Eq. (95) at varying $l_{\max }$. Bayesian statistics uses the volume under the priorweighted likelihood (the Bayesian evidence) as a modelcomparison statistic. However, this typically involves the evaluation of an expensive multidimensional integral, which can sometimes be prohibitive for costly likelihood computations or high-dimensional parameter spaces. Fortunately, mature nested-sampling [54] algorithms, such as MultiNest [55-57], now exist to tackle this problem, and as such, we employ MultiNest in all the following parameter-estimation and evidence recovery calculations.

Our results are shown in Fig. 4, where we see that an expansion up to and including $l=4$ is sufficient to recover the shape of the Hellings and Downs curve. We can show this explicitly by drawing the envelope of overlap reduction functions corresponding to expansion coefficients lying in the $95 \%$ credible interval of the Bayesian analysis. The result is shown in Fig. 5(a), along with the injected Hellings and Downs curve. We also show in Fig. 5(b) that the coefficients of the expansion are consistent with the analytic result of $C_{l}=$ const, $\forall l \geq 2$.

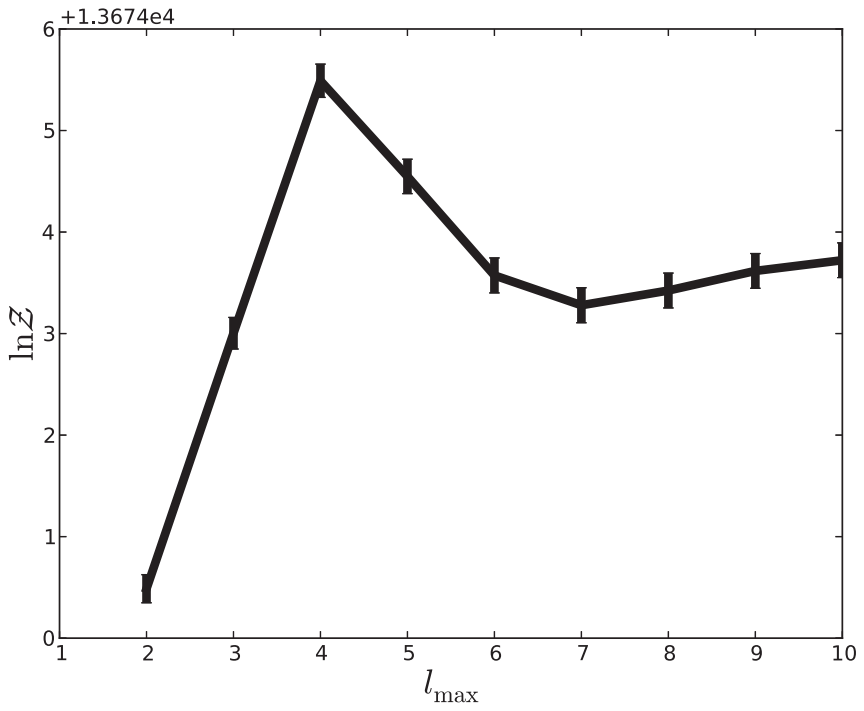

FIG. 4. Bayesian evidence for models of the overlap reduction function truncated at varying $l_{\max }$. To produce this plot, we injected an isotropic, unpolarized and uncorrelated gravitationalwave background with a white-noise spectrum into a set of realistic-format pulsar TOAs. Testing truncated expansions of the form in Eq. (95), and recovering the Bayesian evidence, we find that an expansion up to and including $l=4$ is sufficient to recover the Hellings and Downs curve. 


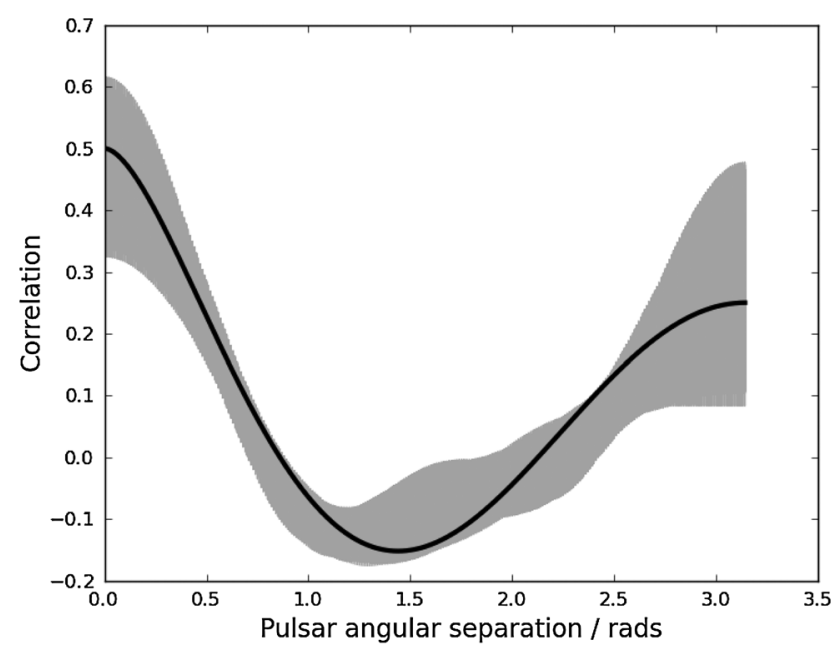

(a)

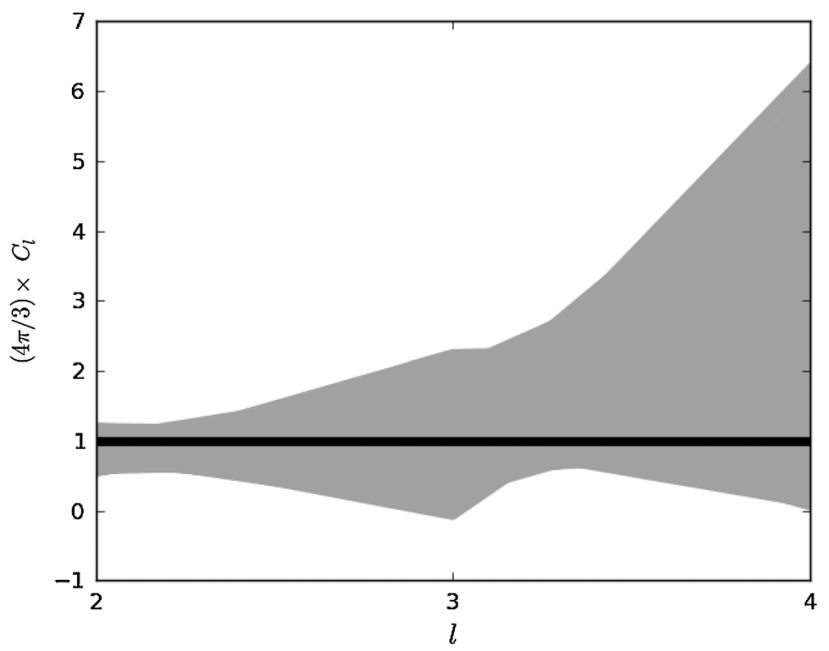

(b)

FIG. 5. In (a) we explicitly show the envelope of overlap reduction functions corresponding to expansion coefficients lying in the 95\% credible interval of the Bayesian analysis of an isotropic, unpolarized and uncorrelated gravitational-wave background. With an expansion up to and including $l=4$, our reconstruction is sufficiently consistent with the injected Hellings and Downs curve. In (b) we demonstrate that our numerical analysis is consistent with the analytic hypothesis that $C_{l}=$ const, $\forall l \geq 2$.

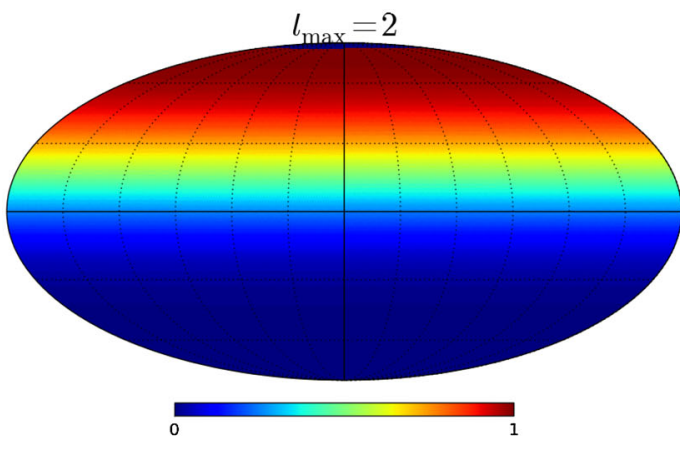

(a)

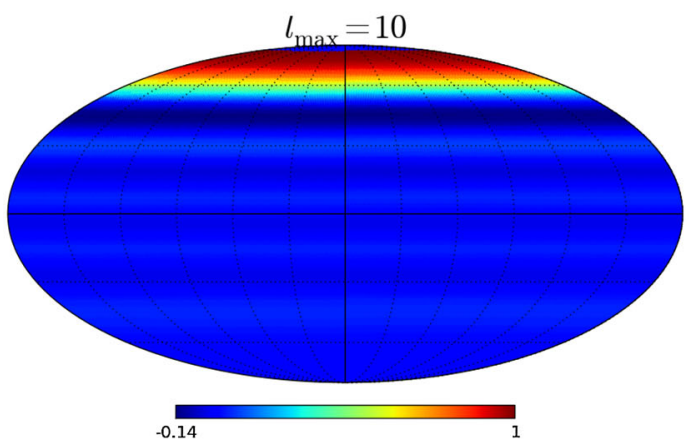

(c)

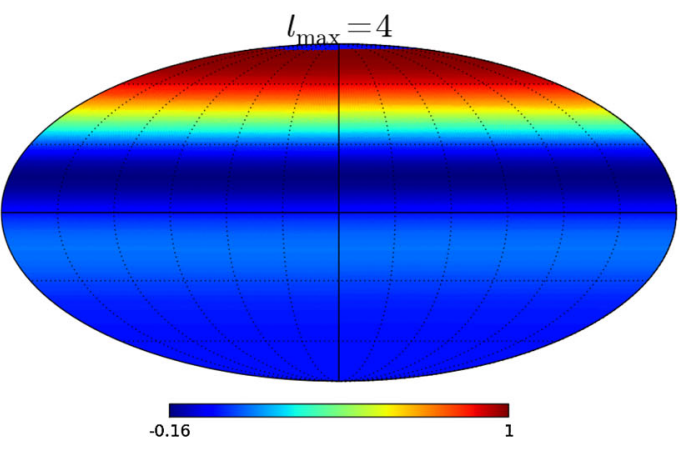

(b)

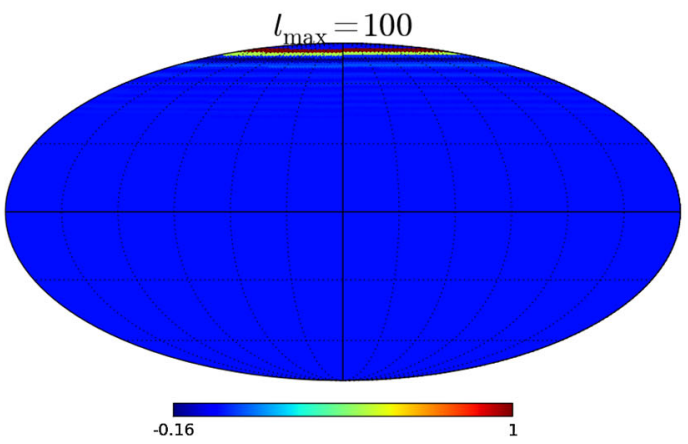

(d)

FIG. 6 (color online). Theoretical correlation maps $\left(\left|\left\langle h_{+}(\hat{k}) h_{+}^{*}\left(\hat{k}^{\prime}\right)\right\rangle_{k}\right|\right)$ showing the degree to which Fourier modes along the $z$ axis and elsewhere on the sky are correlated. An uncorrelated gravitational-wave background should have a delta function in the sky location for the quadratic expectation value of Fourier modes [see Eq. (32)]. As we include higher multipoles in the expansion of Eq. (95), we converge toward this behavior. 


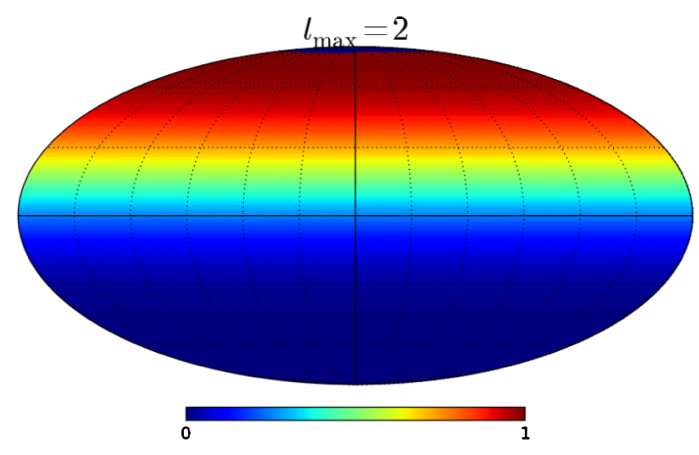

(a)

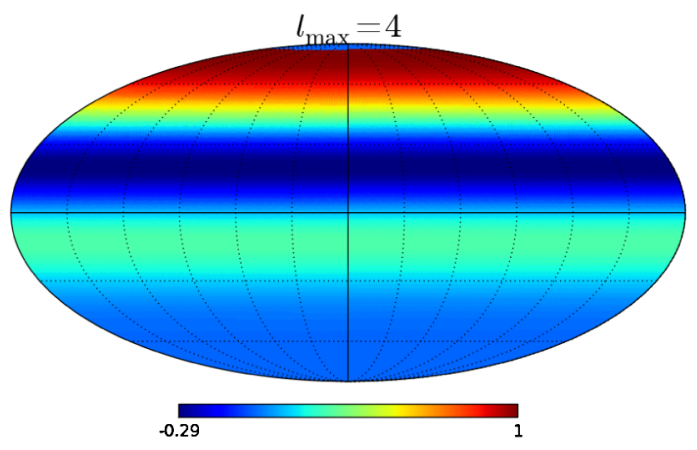

(b)

FIG. 7 (color online). Correlation maps [displaying $\left|\left\langle h_{+}(\hat{k}) h_{+}^{*}\left(\hat{k}^{\prime}\right)\right\rangle_{k}\right|$ ] constructed from the maximum-a posteriori $C_{l}$ values of our Bayesian analysis of a pulsar TOA data set containing an injected isotropic, unpolarized and uncorrelated gravitational-wave background. We see that the truncated expansion of Eq. (95) which gives the highest Bayesian evidence (corresponding to $l_{\text {max }}=4$ ) adequately recovers the restricted sky correlation that is characteristic of an uncorrelated gravitational-wave background.

Furthermore, by expanding to higher multipoles we converge toward the injected case of an uncorrelated background. From Eq. (32) we see that, by definition, an uncorrelated gravitational-wave background should have a delta function in the sky location for the quadratic expectation value of the Fourier amplitudes. We show this explicitly in Fig. 6, where the correlation between Fourier modes along the $z$ axis and elsewhere on the sky are color coded to indicate strength of correlation. For $l_{\max }=100$ the correlation between the Fourier mode along the $z$ axis with other sky locations is almost negligible, effectively giving the delta-function characteristic of an uncorrelated background. From our analysis of the pulsar TOA data set containing an injected uncorrelated gravitational-wave background, we can produce maximum-a posteriori correlation maps from the recovered $C_{l}$ values. In Fig. 7, we see that the expansion with the highest Bayesian evidence (corresponding to $l_{\max }=4$ ) adequately replicates the restricted sky correlation of Fourier modes that is characteristic of an uncorrelated background.

Note that these sky maps of the Fourier-mode correlation are not directly measurable in a PTA analysis - the maps in Fig. 6 were constructed from the theoretical expectation of the correlation of the grad/curl-expanded "plus"/"cross" GW amplitudes for an isotropic background [see Eq. (37) and the discussion in Sec. VI A]. Likewise the maps in Fig. 7 are not directly measurable, but are constructed by inserting the measured coefficients of the overlap reduction function expansion from Eq. (95) into the expressions for the expectation of the Fourier-mode correlations.

\section{CORRELATION FUNCTIONS FOR ARBITRARY BACKGROUNDS}

\section{A. Characterizing anisotropic backgrounds}

The most general form of Eq. (37) that we can write down for the correlation between different modes of the background, while still assuming that different frequency components are uncorrelated and that the frequency and spatial dependence can be factorized is

$$
\left\langle a_{(l m)}^{P}(f) a_{\left(l^{\prime} m^{\prime}\right)}^{P^{\prime} *}\left(f^{\prime}\right)\right\rangle=C_{l m l^{\prime} m^{\prime}}^{P P^{\prime}}(f) \delta\left(f-f^{\prime}\right),
$$

where

$$
C_{l m l^{\prime} m^{\prime}}^{P P^{\prime}}(f)=C_{l m l^{\prime} m^{\prime}}^{P P^{\prime}} H(f) \text {. }
$$

If the background is of cosmological origin, we would impose additional restrictions on the correlation functions. It would be normal to impose statistical isotropy (i.e., no preferred direction on the sky) and possibly requiring parity-invariance or no polarization. This will be discussed in more detail in Sec. VI A. In the following we make no further restrictions on the correlation functions to allow for a completely general background.

For expectation values of the form given in Eq. (106), the correlation between the output of the two detectors given in Eqs. (52)-(54) generalizes to

$$
\left\langle r_{1}(t) r_{2}\left(t^{\prime}\right)\right\rangle=\int_{-\infty}^{\infty} \mathrm{d} f e^{i 2 \pi f\left(t-t^{\prime}\right)} H(f) \Gamma_{12}(f),
$$

where

$\Gamma_{12}(f)=\sum_{(l m)} \sum_{\left(l^{\prime} m^{\prime}\right)} \sum_{P} \sum_{P^{\prime}} C_{l m l^{\prime} m^{\prime}}^{P P^{\prime}} R_{1(l m)}^{P}(f) R_{2\left(l^{\prime} m^{\prime}\right)}^{P^{\prime} *}(f)$,

with $R_{I(l m)}^{P}(f)$ given by Eq. (49). For pulsar timing arrays, the above expression simplifies. As we showed in Sec. III D,

$$
\begin{aligned}
& R_{I(l m)}^{G}(f)=(i 2 \pi f)^{-1} 2 \pi(-1)^{l} N_{l} Y_{(l m)}\left(\hat{u}_{I}\right), \\
& R_{I(l m)}^{C}(f)=0 .
\end{aligned}
$$


Thus,

$$
\begin{aligned}
\Gamma_{12}(f)=f^{-2} \sum_{(l m)} \sum_{\left(l^{\prime} m^{\prime}\right)} & (-1)^{l+l^{\prime}} N_{l} N_{l^{\prime}} C_{l m l^{\prime} m^{\prime}}^{G G} \\
& \times Y_{(l m)}\left(\hat{u}_{1}\right) Y_{\left(l^{\prime} m^{\prime}\right)}^{*}\left(\hat{u}_{2}\right) .
\end{aligned}
$$

A couple of remarks are in order as follows: (i) The overlap reduction function does not depend on $C_{l m l^{\prime} m^{\prime}}^{C C}$, $C_{l m l^{\prime} m^{\prime}}^{G C}$, or $C_{l m l^{\prime} m^{\prime}}^{C G}$. This means that a pulsar timing array is insensitive to the curl modes of the radiation field, no matter how great the amplitude of the curl modes may be. This is a surprising result but arises from the fact that all pulsar lines of sight share a common end, at the SSB, which we have taken to be the origin. We can rotate the axis of the coordinate system used to compute $R_{(l m)}^{P}(f)$ for any given pulsar so that the pulsar is in the $z$ direction and then the response has no dependence on the azimuthal coordinate. For curl modes, in this frame $F^{\times}(\hat{k})=0$, while $F^{+}(\hat{k})$ is multiplied by $X_{(l m)}(\hat{k}) \propto m e^{i m \phi}$. The integral over $\phi$ therefore vanishes for any function that has no azimuthal dependence. A ground-based interferometer such as LIGO that is static also has zero response to curl modes (see Appendix D for a derivation of the response to both grad and curl modes). The reason that we have no sensitivity to curl modes is that the metric perturbation for these modes vanishes at the origin of coordinates. This is somewhat analogous to a separation between even and odd modes. If we have waves propagating in opposite directions in one dimension, a measurement at the origin can only determine half of the parameters characterizing the waves since odd modes are always zero there. If we have a pointable detector, we can recover all the modes by pointing the detector first to the left and then to the right, and we can also do this by adding a measurement at a second point. (CMB temperature and polarization measurements, for example, are made with detectors that are sensitive to only a small fraction of the sky.) Gravitational-wave detectors are not pointable, but if we had a network of spatially separated detectors we should have sensitivity to these modes as the origin can be taken to be at the location of one detector only. Mathematically, shifting the SSB to the point $\vec{x}_{0}$ introduces an extra term $\exp \left[-i 2 \pi f \hat{k} \cdot \vec{x}_{0} / c\right]$ in the response, which breaks the azimuthal symmetry. Physically, although curl modes are transformed to curl modes under rotations, a translation mixes curl and grad modes, which leads to this nonzero response in the shifted reference frame.

The symmetry is also broken by detector motion. Groundbased interferometers move due to both the rotation of Earth and the orbital motion of Earth around the Sun. Pulsartiming arrays use radio telescopes based on Earth which also move. However, there are two fundamental differences between PTAs and ground-based interferometers. First, the frequency ranges are very different. The light-travel time from Earth to the Sun is $~ 500 \mathrm{~s}$ while the maximum frequency a PTA is sensitive to is $\sim 10^{-6} \mathrm{~Hz}$ (assuming observations approximately every two weeks). The value of $f \hat{k} \cdot \vec{x} / c$ therefore varies by less than 0.0005 for a PTA. For LIGO the maximum frequency is $\sim 1 \mathrm{kHz}$, and over a year $f \hat{k} \cdot \vec{x} / c$ varies by $5 \times 10^{5}$ due to the motion of Earth around the Sun, and by an additional $\sim 20$ on a daily basis due to Earth's rotation. Even for the lowest frequencies $(f \sim 10 \mathrm{~Hz})$ that LIGO can detect, the daily variation from Earth's orbital motion is $\gtrsim 10$. The second difference is in the nature of the data. For a PTA the raw data are the arrival times of pulses from given pulsars at the detector. This is typically (and straightforwardly) converted into an arrival time at the SSB, which is a fixed origin. For ground-based interferometers, the measurement is of a path-length difference between two arms with a particular instantaneous orientation, which cannot be readily converted into the response of a fixedorientation detector at some other point. We conclude that PTAs really are static point detectors with no response to curl modes, while ground-based interferometers will be able to measure these modes in practice as a result of the motion of the detectors. The energy density in the gravitational-wave background is given by the sum of the squares of the grad and curl amplitudes, so the curl component is a real physical part of the background to which PTAs are insensitive. (Note that including the pulsar term does not change this conclusion as it is also axisymmetric in the frame in which the pulsar position is along the $z$ axis.)

(ii) We can recover Eq. (83) for the overlap reduction function for a statistically isotropic background with $C_{l}^{G G}=C_{l}^{C C} \equiv C_{l}$ and $C_{l}^{G C}=0=C_{l}^{C G}$ by simply setting $C_{l m l^{\prime} m^{\prime}}^{G G}=C_{l} \delta_{l l^{\prime}} \delta_{m m^{\prime}}$. The insensitivity of a PTA to curl modes is irrelevant when searching for such a background, since all the information about the correlation structure of the background is contained in $C_{l}^{G G}$. (This will be discussed in more detail in Sec. V D.) Making the above substitution for $C_{l m l^{\prime} m^{\prime}}^{G G}$ into Eq. (111) and using the addition theorem for (ordinary) spherical harmonics, we obtain Eq. (83) with $\cos \zeta=\hat{u}_{1} \cdot \hat{u}_{2}$.

\section{B. Representation of anisotropic uncorrelated backgrounds}

Equations (33) and (35) give the correlation functions that have to be imposed on the $a_{(l m)}^{P}(f)$ 's in order to recover an isotropic, unpolarized and uncorrelated background. If instead we want to represent an unpolarized, uncorrelated but anisotropic background, then we require

$$
\begin{aligned}
&\left\langle h_{+}(f, \hat{k}) h_{+}^{*}\left(f^{\prime}, \hat{k}^{\prime}\right)\right\rangle=\left\langle h_{\times}(f, \hat{k}) h_{\times}^{*}\left(f^{\prime}, \hat{k}^{\prime}\right)\right\rangle=\frac{1}{2} P(\hat{k}) H(f) \delta^{2}\left(\hat{k}, \hat{k}^{\prime}\right) \delta\left(f-f^{\prime}\right), \\
&\left\langle h_{+}(f, \hat{k}) h_{\times}^{*}\left(f^{\prime}, \hat{k}^{\prime}\right)\right\rangle=\left\langle h_{\times}(f, \hat{k}) h_{+}^{*}\left(f^{\prime}, \hat{k}^{\prime}\right)\right\rangle=0,
\end{aligned}
$$


where $P(\hat{k})$ defines the anisotropic distribution of gravitational-wave power on the sky. [We are assuming here that the spectral and angular dependence of the background factorize as $P(\hat{k}) H(f)$.] Since an arbitrary scalar function $P(\hat{k})$ can be written as a linear combination of spherical harmonics, we consider a single term in this sum, $Y_{L M}(\hat{k})$. Using Eq. (31) to solve for $a_{(l m)}^{G}(f)$ and $a_{(l m)}^{C}(f)$, we find

$$
\begin{aligned}
\left\langle a_{(l m)}^{G}(f) a_{\left(l^{\prime} m^{\prime}\right)}^{G *}\left(f^{\prime}\right)\right\rangle & =\left\langle a_{(l m)}^{C}(f) a_{\left(l^{\prime} m^{\prime}\right)}^{C *}\left(f^{\prime}\right)\right\rangle \\
& =\frac{1}{2} \int \mathrm{d}^{2} \Omega_{\hat{k}} Y_{L M}(\hat{k})\left({ }_{2} Y_{(l m)}^{*}(\hat{k})_{2} Y_{\left(l^{\prime} m^{\prime}\right)}(\hat{k})+_{-2} Y_{(l m)}^{*}(\hat{k})_{-2} Y_{\left(l^{\prime} m^{\prime}\right)}(\hat{k})\right) H(f) \delta\left(f-f^{\prime}\right), \\
\left\langle a_{(l m)}^{G}(f) a_{\left(l^{\prime} m^{\prime}\right)}^{C *}\left(f^{\prime}\right)\right\rangle & =-\left\langle a_{(l m)}^{C}(f) a_{\left(l^{\prime} m^{\prime}\right)}^{G *}\left(f^{\prime}\right)\right\rangle \\
& \left.=\frac{i}{2} \int \mathrm{d}^{2} \Omega_{\hat{k}} Y_{L M}(\hat{k}){ }_{2} Y_{(l m)}^{*}(\hat{k})_{2} Y_{\left(l^{\prime} m^{\prime}\right)}(\hat{k})-_{-2} Y_{(l m)}^{*}(\hat{k})_{-2} Y_{\left(l^{\prime} m^{\prime}\right)}(\hat{k})\right) H(f) \delta\left(f-f^{\prime}\right) .
\end{aligned}
$$

The integrals can be written in terms of Wigner 3-j symbols (see for example [58,59]),

$$
\begin{aligned}
\left\langle a_{(l m)}^{G}(f) a_{\left(l^{\prime} m^{\prime}\right)}^{G *}\left(f^{\prime}\right)\right\rangle & =\left\langle a_{(l m)}^{C}(f) a_{\left(l^{\prime} m^{\prime}\right)}^{C *}\left(f^{\prime}\right)\right\rangle \\
& =\frac{(-1)^{m}}{2} \sqrt{\frac{(2 L+1)(2 l+1)\left(2 l^{\prime}+1\right)}{4 \pi}}\left(\begin{array}{ccc}
L & l & l^{\prime} \\
M & -m & m^{\prime}
\end{array}\right)\left[\left(\begin{array}{ccc}
L & l & l^{\prime} \\
0 & 2 & -2
\end{array}\right)+\left(\begin{array}{ccc}
L & l & l^{\prime} \\
0 & -2 & 2
\end{array}\right)\right] H(f) \delta\left(f-f^{\prime}\right), \\
\left\langle a_{(l m)}^{G}(f) a_{\left(l^{\prime} m^{\prime}\right)}^{C *}\left(f^{\prime}\right)\right\rangle & =-\left\langle a_{(l m)}^{C}(f) a_{\left(l^{\prime} m^{\prime}\right)}^{G *}\left(f^{\prime}\right)\right\rangle \\
& =\frac{i(-1)^{m}}{2} \sqrt{\frac{(2 L+1)(2 l+1)\left(2 l^{\prime}+1\right)}{4 \pi}}\left(\begin{array}{ccc}
L & l & l^{\prime} \\
M & -m & m^{\prime}
\end{array}\right)\left[\left(\begin{array}{ccc}
L & l & l^{\prime} \\
0 & 2 & -2
\end{array}\right)-\left(\begin{array}{ccc}
L & l & l^{\prime} \\
0 & -2 & 2
\end{array}\right)\right] H(f) \delta\left(f-f^{\prime}\right) .
\end{aligned}
$$

Explicit expressions for the Wigner 3-j symbols are given in Appendix A.

It is clear from the above that the representation of an anisotropic background using this formalism is quite complicated. However, that is also the case for the standard approach $[34,35]$ to searching for an anisotropic uncorrelated background. The spherical harmonic components of the frequency-independent part of the overlap reduction function for such a background are given by [34]

$$
\bar{\Gamma}_{12, l m}=\int_{S^{2}} \mathrm{~d}^{2} \Omega_{\hat{k}} Y_{l m}(\hat{k})\left[F_{1}^{+}(\hat{k}) F_{2}^{+}(\hat{k})+F_{1}^{\times}(\hat{k}) F_{2}^{\times}(\hat{k})\right],
$$

where $l=0,1,2, \ldots$. These integrals can be evaluated using techniques similar to those used in Sec. III C. This is described in detail in Appendix E. There we derive analytic expressions for $\bar{\Gamma}_{12, l m}$ for all values of $l$ and $m$, extending the analytical results of [34]. Figure 8 shows plots of $\bar{\Gamma}_{12, l m}$ for $l=0,1, \ldots, 5$, calculated using formulas from Appendix E. The plots for $l=0,1,2,3$ are identical to those in Fig. 2 of [34], as expected. We will not consider anisotropic uncorrelated backgrounds further in this paper, but refer the reader to $[34,35]$ where these are considered in depth.

\section{MAPPING THE GRAVITATIONAL-WAVE BACKGROUND}

In the preceding sections we showed how this formalism can be used to describe two specific models for the gravitational-wave background. However, the advantage of this approach is that it allows us to characterize the background and produce a map of it, without making any assumptions about its underlying nature. As described below, we will be able to produce a map of both the amplitude and the phase of the gravitational-wave background as a function of position on the sky. This map will have a resolution limited by the number of pulsars in the array, and in general we will measure the small- $l$ (large angular scale) components more accurately, so we expect the resolution of the map to increase as more pulsars are added. This map encodes all the information that it is possible to extract from our data about the gravitationalwave background. The power distribution on the sky will identify hot spots where there is enhanced gravitationalwave emission, which might correspond to nearby individual sources or even clusters containing multiple sources. In addition, the variation of the phase across the sky will indicate whether there is any correlation between the emission at different sky locations. The amount of information that can be extracted will of course depend on the 


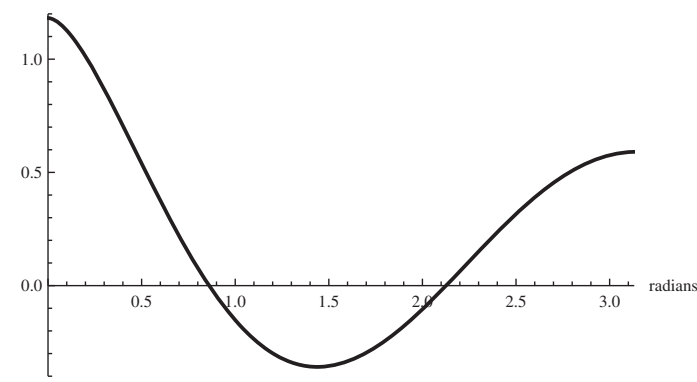

(a)

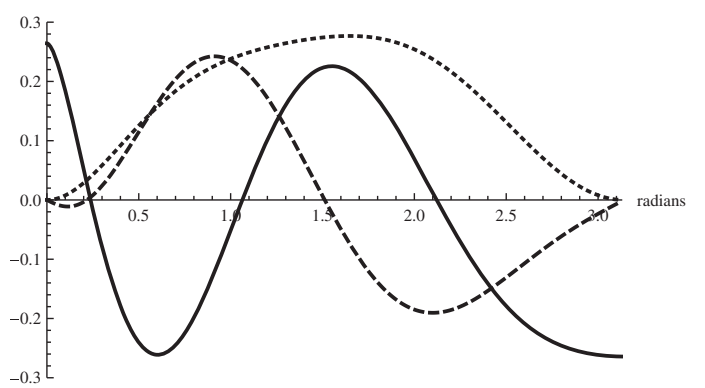

(c)

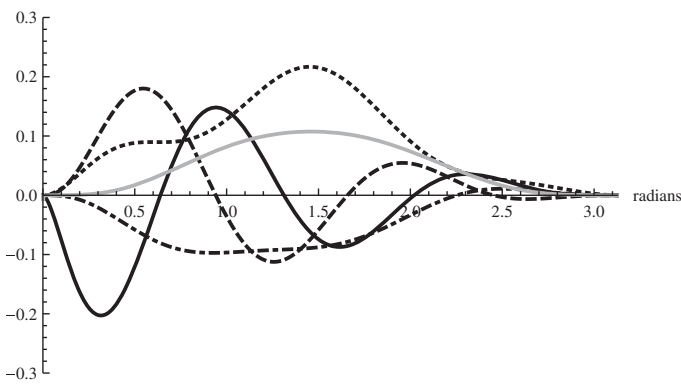

(e)

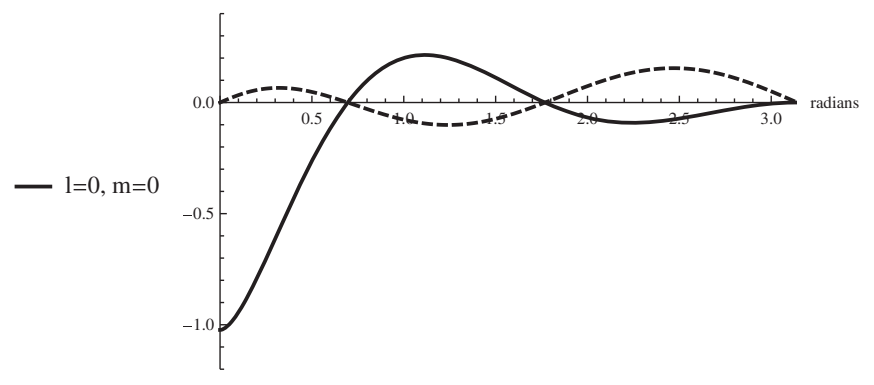

$\begin{array}{rl}-1 & 1, m=0 \\ ---1=1, m=1\end{array}$

(b)

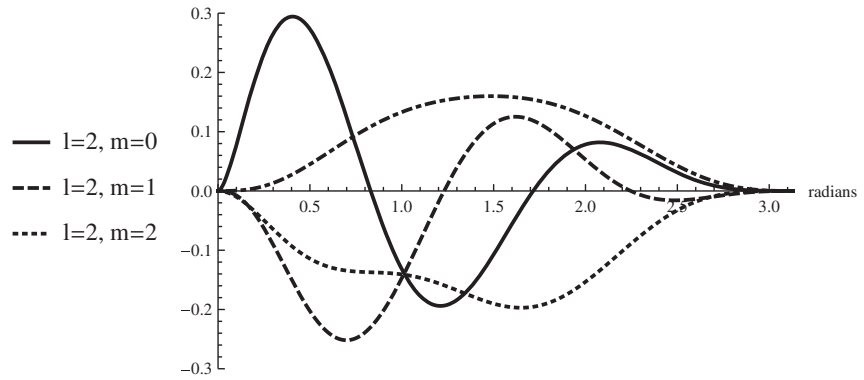

$-1=3, m=0$
$-\cdots 1=3, m=1$
$\cdots \quad l=3, m=2$
$\cdots 1=3, m=3$

(d)

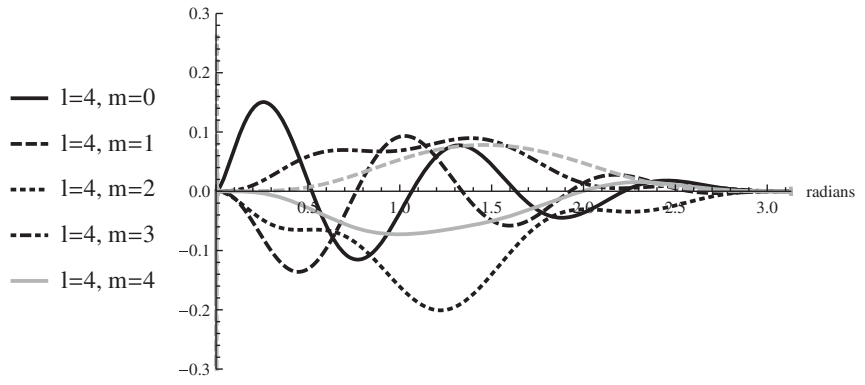

$\begin{aligned} &-1=5, m=0 \\ &--1=5, m=1 \\ & \cdots 1=5, m=2 \\ &-\cdots 1=5, m=3 \\ &-1=5, m=4 \\ &--1=5, m=5\end{aligned}$

(f)

FIG. 8. Plots of $\bar{\Gamma}_{12, l m}$ for $l=0,1, \ldots, 5$ as a function of the angle between the two pulsars for an anisotropic, unpolarized and uncorrelated background.

resolution of the map that we can measure. The expectation is that the background will be isotropic, unpolarized and uncorrelated, and we saw above that we would need to measure components up to $l_{\max }=4$ to recover the Hellings and Downs curve characteristic of that case. Our ability to identify departures from this model will depend on the size of those departures. If the departures are large, they will become apparent much more quickly, perhaps even $l_{\max }=3$ would be sufficient, but for small departures we would require many more components. To identify hot spots we would need an angular resolution comparable to the size of the hot spot. Taking the latter to be the typical resolution of an individual source on the sky can be used to set requirements on $l_{\max }$ and hence the number of pulsars. We will discuss all of these considerations in this section.

\section{A. Likelihood function for the $a_{(l m)}^{G}$ 's}

By measuring the coefficients $a_{(l m)}^{P}(f)$ using a pulsar timing array we can reconstruct a map of the gravitationalwave sky, or at least that part of it, spanned by the gradient modes $a_{(l m)}^{G}(f)$, which is visible to a PTA. A typical PTA will consist of measured timing residuals $\delta t_{I}(t)$ for a set of $N$ pulsars, labeled by $I$. The timing residuals will be a combination of a gravitational-wave signal component and a noise component

$$
\delta t_{I}(t)=r_{I}(t)+n_{I}(t), \quad \tilde{\delta} t_{I}(f)=\tilde{r}_{I}(f)+\tilde{n}_{I}(f),
$$

where $I=1,2, \ldots, N$ and the tilde denotes Fourier transform. Usually the stochastic background is taken to be part of the noise, but in the approach described here we are using the decomposition into grad and curl modes to construct a template for the gravitational-wave background; therefore it is the signal component of the timing residuals. In the Fourier domain we have [cf. Eqs. (48) and (49)]

$$
\tilde{r}_{I}(f) \equiv \tilde{r}_{I}(f ; \vec{a})=\sum_{(l m)} R_{I(l m)}^{G}(f) a_{(l m)}^{G}(f),
$$


where $\vec{a} \equiv\left\{a_{(l m)}^{G}(f)\right\}$ is shorthand notation for the collection of parameters describing the gravitational-wave background. Assuming stationary and Gaussian noise, but allowing for possible correlated noise between different pulsars, we have

$$
\left\langle\tilde{n}_{I}(f) \tilde{n}_{J}^{*}\left(f^{\prime}\right)\right\rangle=C_{n I J}(f) \delta\left(f-f^{\prime}\right),
$$

and the likelihood of measuring the data $\tilde{\delta} t_{I}(f)$ is then

$$
p\left(\delta t \mid C_{n}, \vec{a}\right) \propto \exp \left\{-\frac{1}{2} \int_{-\infty}^{\infty} \mathrm{d} f\left[\left(\tilde{\delta} t_{I}^{*}(f)-\tilde{r}_{I}^{*}(f ; \vec{a})\right) C_{n I J}^{-1}(f)\left(\tilde{\delta} t_{J}(f)-\tilde{r}_{J}(f ; \vec{a})\right)\right]\right\},
$$

where summation over the pulsar indices $I, J$ is assumed. The values of the coefficients $a_{(l m)}^{G}(f)$ can then be recovered either by using Bayesian inference to construct their posterior distributions given prior probability distributions for the signal and noise parameters or by computing the frequentist maximum-likelihood estimators of the parameters, which we will illustrate below.

As written above there are an uncountable number of model parameters, since we not only have a sum over different $(\mathrm{lm})$ modes of the background, but we are allowing the coefficients $a_{(l m)}^{G}(f)$ to be arbitrary functions of frequency. To make progress there are several possible approaches: (i) we assume that the $a_{(l m)}^{G}(f)$ are independent of frequency, for instance by filtering the data so that we consider only a narrow range of frequencies for which this would be a reasonable approximation; (ii) we assume that the frequency and spatial dependence of the coefficients factorize, $a_{(l m)}^{G}(f)=\sqrt{H(f)} a_{(l m)}^{G}$; (iii) we assume the background consists of a finite number of components only, with frequencies $f_{j}$. In approach (iii) the included frequency components could be the entire set of discrete frequencies to which we are sensitive with a finite data set, or a smaller number of components. It has been shown in the context of the International Pulsar Timing Array (IPTA) mock data challenge that an isotropic stochastic background can be well represented as a superposition of a small number of components in this way [60]. All three of these approaches are mathematically equivalent in the sense that they reduce the number of $a_{(l m)}^{G}(f)$ coefficients to a finite value for a given $(\mathrm{Im})$, but we will use approach (iii) to illustrate how to obtain the maximum-likelihood estimators of the $a_{(l m)}^{G}(f)$ 's in the following.

If we make this assumption and also assume the data set is finite so that the integral over frequency in Eq. (119) reduces to a sum, then we can introduce a new signal vector which combines the response of all the pulsars

$$
\vec{\delta} t \equiv\left\{\delta t_{i}\right\}^{T} \equiv\left\{\tilde{\delta} t_{1}\left(f_{1}\right), \tilde{\delta}_{1}\left(f_{2}\right), \ldots, \tilde{\delta} t_{N}\left(f_{n}\right)\right\}^{T},
$$

where $i=1,2, \ldots, n N$ labels a component of $\vec{\delta} t$, and $j=1,2, \ldots, n$ labels a frequency component $f_{j}$ to which we are sensitive. The (symmetric) $n N \times n N$ correlation matrix for the whole signal then takes the form

$$
F_{i i^{\prime}}=C_{n I I^{\prime}}\left(f_{j}\right) \delta_{j j^{\prime}}
$$

where $j=\bmod (i, n)$ labels a discrete frequency and $I=$ $\lceil i / n\rceil$ labels a pulsar. (Here $\lceil X\rceil$ denotes the smallest integer greater than $X$.) Similarly, the response of the pulsar timing array to the background can be written as $H \vec{a}$, where

$$
H_{i k}=R_{I(l m)_{k}}^{G}\left(f_{j_{k}}\right) \delta_{j j_{k}}
$$

and

$$
\vec{a} \equiv\left\{a_{k}\right\}^{T} \equiv\left\{a_{(l m)_{k}}^{G}\left(f_{j_{k}}\right)\right\}^{T} .
$$

Here $k$ labels a particular component of the signal parameter vector $\vec{a}$, which is specified by spherical harmonic indices $(\operatorname{lm})_{k}$ and discrete frequency index $j_{k}$. Note that each column of $H$ corresponds to a single component of $\vec{a}$ and describes the frequency-domain response of the PTA network to the corresponding mode of the background. Using this notation the likelihood takes the general form

$$
p(\delta t \mid F, \vec{a}) \propto \exp \left[-\frac{1}{2}(\vec{\delta} t-H \vec{a})^{\dagger} F^{-1}(\vec{\delta} t-H \vec{a})\right] .
$$

The reason for introducing this notation is that, in practice, PTA data are measured in the time domain and are not evenly sampled, which makes analyzing the data in the frequency domain as described above quite challenging. In addition, a timing model is fitted out for each pulsar in the array to account for various astrophysical effects including the proper motion of the pulsar, its spin-down and possible binary parameters [61]. This timing-model fit can also affect the gravitational-wave signal in the data. Accounting for this by introducing timing-model parameters into the likelihood and then marginalizing over them results in a modified likelihood that takes exactly the same form as Eq. (124). The measured data $\vec{\delta} t$ are again the concatenation of the measured residuals from the pulsars in the array, the model matrix $H$ now contains the timedomain response of the PTA to the modes of the background, and the correlation matrix takes the form $F^{-1}=G\left(G^{T} C_{n} G\right)^{-1} G^{T}$, where $C_{n}$ is the time-domain correlation matrix for the noise and $G$ is constructed from 
the left null space of the timing-model design matrix, where it effectively projects the timing residuals into a space orthogonal to the timing model [53].

\section{B. Maximum-likelihood estimates of the gravitational-wave sky}

Starting from a likelihood of the form given in Eq. (124), it is relatively simple to compute the frequentist maximumlikelihood estimators of the signal parameters since they enter the model linearly. We find

$$
\left.\vec{a}\right|_{\mathrm{ML}}=\left(H^{\dagger} F^{-1} H\right)^{-1} H^{\dagger} F^{-1} \vec{\delta} t,
$$

assuming that the vector $\vec{\delta} t$ and matrix $F$ are both real. Once these maximum-likelihood estimators or posterior distributions for the $\vec{a}$ parameters have been obtained, a sky map of the gravitational-wave background at each frequency $f_{j}$ can be constructed using Eq. (27) and, in the Bayesian case, integrating over the posterior distribution of $\vec{a}$. The matrix $H^{\dagger} F^{-1} H$ that enters the above expression is the Fisher information matrix, and we have assumed it is invertible, but this will not necessarily be the case. Although we have reduced the number of $a_{(I m)}^{G}(f)$ coefficients by assuming a finite number of frequency components, there are still an infinite number of coefficients as we vary over $l$ and $m$. In practice we will not be able to measure all of the coefficients as we have only a finite amount of noisy data and, most importantly, a finite number of pulsars. Each pulsar will allow us to measure the amplitude of a sine and a cosine quadrature at a particular frequency. As the array is static we would therefore expect to be able to measure only $2 N$ real components of the background at any given frequency.

That this is the number of modes that can be resolved can be understood mathematically by considering the structure of the matrix $H$. Up until now we have allowed $H$ and $\vec{a}$ to be arbitrary complex quantities, but the measured data in the time domain must be real. As we saw in Eq. (13), the negative-frequency components of $a_{(l m)}^{G}(f)$ must satisfy the constraint

$$
a_{(l m)}^{G}(-f)=(-1)^{m} a_{(l,-m)}^{G *}(f) .
$$

In addition, Eqs. (110) and (A5) imply that $R_{(l m)}^{G}(-f)=$ $(-1)^{m} R_{(l,-m)}^{G *}(f)$, and therefore this constraint is sufficient to make $H \vec{a}$ a real time series. Including only positivefrequency components in both $H$ and $\vec{a}$, the likelihood takes the same form, but with $H \vec{a}$ replaced by $\left(H \vec{a}+H^{*} \vec{a}^{*}\right)$. Alternatively, we can replace $\vec{a}$ by a real vector with twice as many components, with alternate entries being the real and imaginary parts of the complex $a_{(l m)}^{G}(f)$ components, and similarly double the number of columns of $H$, making it into a real matrix.
If we suppose that we have included $N_{m}$ modes in $\vec{a}$, and consider a single frequency component $f_{j}$ for simplicity, then, in the time domain, the matrix $H$ has the form

$$
H_{i k}=\sum_{I=1}^{N} x_{I i} R_{I(l m)_{k}}^{G}\left(f_{j}\right),
$$

in which $\left\{x_{I i}\right\}$ is the (complex) time series of pulsar $I$ for the given frequency component $f_{j}$ (i.e., $\exp \left[i 2 \pi f_{j} t\right]$ evaluated at the appropriate discrete times for that pulsar) and has zeros in all components that correspond to samples from the other pulsars. [Note that for the time-domain analysis, the index $i=1,2, \ldots, M$ now labels the times for all the pulsars in the array, and $k$, as before, labels a particular component of the signal parameter vector $\vec{a}$, having spherical harmonic indices $(l m)_{k}$ and frequency $f_{j_{k}}=f_{j}$ for this particular case.] It is clear that the nontrivial left eigenspace of $H$ (the range of $H$ ) is spanned by the $N$ complex vectors $\vec{x}_{I} \equiv\left\{x_{I i}\right\}$ (equivalently, the columns of $H$ are linear combinations of these $N$ complex vectors) and that there are therefore $N_{m}-N$ complex left null eigenvectors. If we do a singular-value decomposition

$$
H=U \Sigma V^{\dagger},
$$

then the $M \times N_{m}$ rectangular matrix $\Sigma$ will have at most $N$ nonzero elements on its diagonal, $\sigma_{i} \equiv \Sigma_{i i}$. (For this form of singular-value decomposition, $U$ and $V$ are unitary matrices having dimensions $M \times M$ and $N_{m} \times N_{m}$, respectively.) This means that we will have sensitivity to $N$ complex combinations of the $\vec{a}$ coefficients and that there will be an additional $N_{m}-N$ combinations which we cannot detect. In the real representation, $H$ has twice as many columns and there will be twice as many nonzero singular values, essentially corresponding to the real and imaginary parts of the columns of $U$. For a given column $\vec{v}_{i}$ of $V$ and $\vec{u}_{i}$ of $U$, we have $H \vec{v}_{i}=\sigma_{i} \vec{u}_{i}$. The vectors $\vec{v}_{i}$ and $-i \vec{v}_{i}$ give the two corresponding singular vectors of $\left(H \vec{a}+H^{*} \vec{a}^{*}\right)$, which map onto real vectors that are twice the real and twice the imaginary parts of the complex vector $\sigma_{i} \vec{u}_{i}$.

We can split the columns of the matrix $U$ as $U=\left[H_{\text {range }} H_{\text {null }}\right]$, where $H_{\text {range }}$ represents the first $N$ columns of $U$ which span the range of $H$. We must then replace $H \vec{a}$ by $H_{\text {range }} \vec{b}$ in the likelihood, Eq. (124), and can proceed as before. Once the maximum-likelihood estimator of $\vec{b}$ is obtained we get the maximum-likelihood value of $\vec{a}$, projected into the space to which we have sensitivity, by computing $\vec{a}=V \Sigma^{+} \vec{b}$, where $\Sigma^{+}$is the pseudoinverse of $\Sigma$, obtained by replacing the nonzero elements on the diagonal of $\Sigma$ by their reciprocals and taking the transpose of the resulting matrix.

As this work was nearing completion, we became aware of an ongoing independent study by Cornish and van Haasteren (private communication; this work has subsequently appeared as [62]) that is also concerned with 
PTA mapping of the gravitational-wave background. Their approach is based on a real-space description of the background using pixelized sky maps, and they have found that an $N$-pulsar array is sensitive to $2 N$ different "sky map basis" elements. These correspond to the $N$ complex combinations of $a_{(l m)}^{G}(f)$ modes to which we find a PTA is sensitive. The Cornish and van Hassteren work will provide a more detailed study of sky mapping, demonstrating the practical application of ideas similar to those discussed in this section, while the theoretical results described elsewhere in this paper provide a way to interpret such results and an understanding of the physical nature of the individual sky map basis elements. In addition, our results indicate that there is a portion of the gravitationalwave sky that a pulsar timing array will never be able to see. At fixed resolution (which means a fixed pixel size in a real-space representation or a fixed number of $l$ modes in our representation) you would expect to be able to measure all of the background components once you had more pulsars in the array than components in the finite resolution representation. However, this is not the case. While it is possible to measure the whole grad component of the sky at a fixed resolution once sufficiently many pulsars are included in the array, the array is always blind to the curl component.

Increasing the number of frequency components does not provide sensitivity to additional components of the background. When adding another frequency, we can simply add another set of $a_{(l m)}^{G}$ 's at the new frequency, in which case we will be able to determine $N$ of these coefficients for each frequency component. Alternatively, we can assume that the new $a_{(l m)}^{G}$ 's are equal to the first set of $a_{(l m)}^{G}$ 's, or equal to a frequency-dependent multiple of the first set. In that case we still cannot measure any additional combinations of $a_{(l m)}^{G}$ 's, but the additional frequency components will allow us to measure the same combinations of coefficients with greater precision.

\section{Example recovery of a simulated background}

We now illustrate the mapping procedure described above by constructing maximum-likelihood sky maps of $h_{+}(f, \hat{k})$ and $h_{\times}(f, \hat{k})$ for a simulated gravitational-wave background. For simplicity, we consider only a single frequency component and a noise-free simulation. [So we will drop the $f$ from $h_{+, \times}(f, \hat{k})$ and simply write $h_{+, \times}(\hat{k})$ in what follows.] More realistic simulations will be presented in a detailed follow-up paper to Ref. [62]. Mollweide projections of the real part of $h_{+}(\hat{k})$ for the simulated background and its gradient and curl components are shown in Fig. 9. The sky map for the simulated background shown in panel (a) is the same as the real part of $h_{+}(\hat{k})$ in the top-left panel of Fig. 3. [The imaginary part of $h_{+}(\hat{k})$ and the real and imaginary parts of $h_{\times}(\hat{k})$ for the simulated background are qualitatively similar and are shown in three other panels in Fig. 3.] The simulated gradient component shown in Fig. 9(b) is for a statistically isotropic background with $C_{l}=1$ for $l=2,3, \ldots, 10$; and the simulated curl component shown in Fig. 9(c) is also for a statistically isotropic background, but with $C_{l}=4$ for $l=2,3,4,5$. (The smaller value of $l_{\max }$ for the curl component is responsible for the larger angular-scale structure in the curl map, and the larger values for the $C_{l}$ 's for the curl component were chosen to make its contribution to the total simulated background comparable to that of the grad component.) The total simulated background in Fig. 9(a) is just the sum of the gradient and curl components.

Figure 10 shows the results of the maximum-likelihood estimation of the simulated background for PTAs containing different numbers of pulsars. Mollweide projections of the real part of $h_{+}(\hat{k})$ are shown for the gradient component of the simulated background (first column), the maximumlikelihood recovered sky maps (second column), and the residual sky maps (third column). The residual maps are the difference between the gradient component of the simulated background and the recovered maps. The six rows correspond to PTAs containing $N=1,5,10,20,50$, and 100 pulsars, respectively. The maximum-likelihood estimates of the spherical harmonic components are constructed out to $l_{\max }=10$. The size of the pixels used for the sky maps corresponds to an angular resolution of $\Delta \Omega \sim 50 \mathrm{deg}^{2}$, which is much finer than that achievable

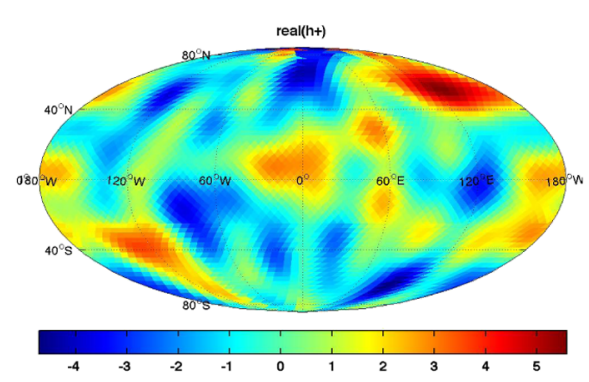

(a) Total map (grad+curl)

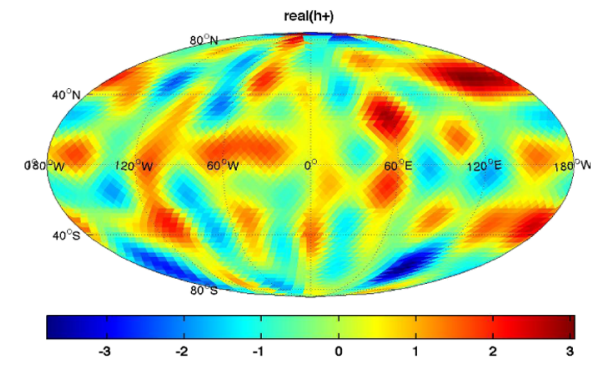

(b) Gradient component

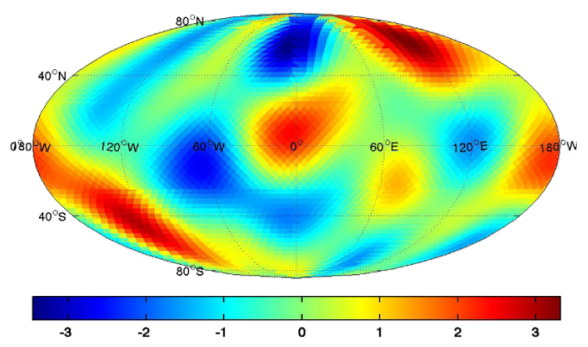

(c) Curl component

FIG. 9 (color online). Mollweide projections of $\operatorname{Re}\left(h_{+}\right)$for the simulated gravitational-wave background. Panel (a) shows the total simulated background (grad+curl components); panel (b) shows the gradient component; and panel (c) shows the curl component. Sky maps of $\operatorname{Im}\left(h_{+}\right), \operatorname{Re}\left(h_{\times}\right)$, and $\operatorname{Im}\left(h_{\times}\right)$are similar. 

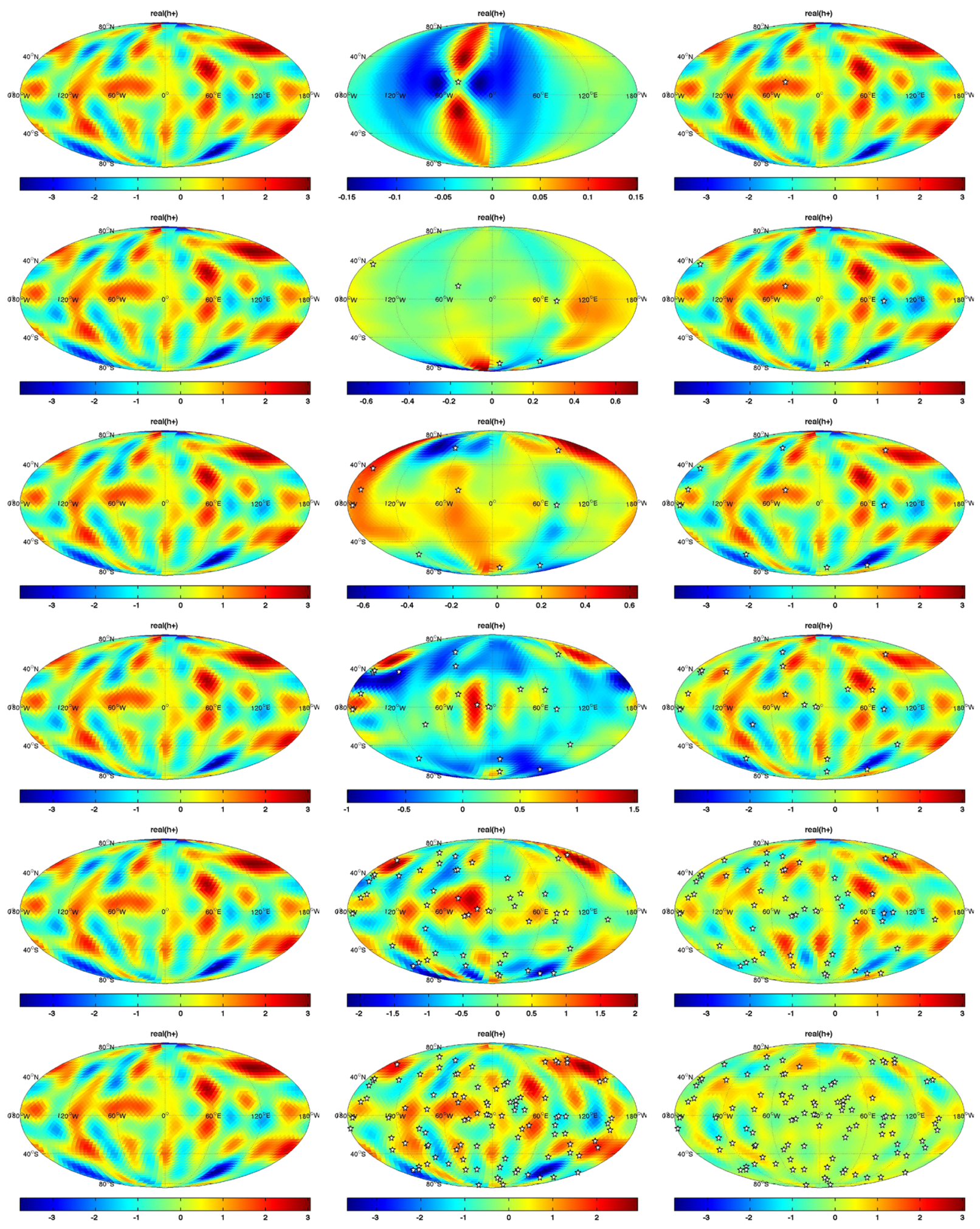

FIG. 10 (color online). Mollweide projections of $\operatorname{Re}\left(h_{+}\right)$for the grad component of the simulated background (first column), the maximum-likelihood recovered sky maps (second column), and residual sky maps (third column) for PTAs containing different numbers of pulsars. The residual sky maps are the difference between the grad component of the simulated background and the recovered maps. Maps of $\operatorname{Im}\left(h_{+}\right), \operatorname{Re}\left(h_{\times}\right)$, and $\operatorname{Im}\left(h_{\times}\right)$are similar. The rows correspond to PTAs containing $N=1,5,10,20,50$, and 100 pulsars, respectively. The pulsar locations are shown as white stars. We used a variable color scale in the recovered maps to better show the angular structure in the small- $N$ maps, which would not have been visible if we had used the same (fixed) color scale used to make the simulated background and residual maps. 


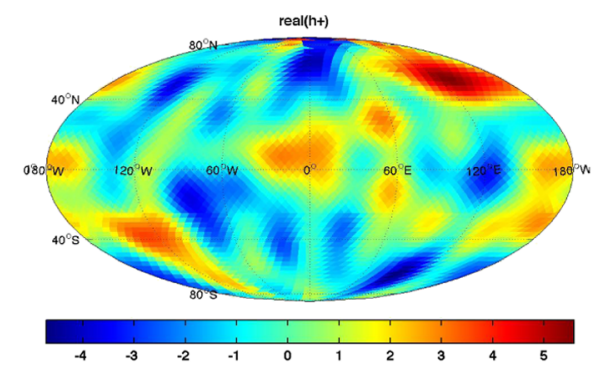

(a) Total map (grad+curl)

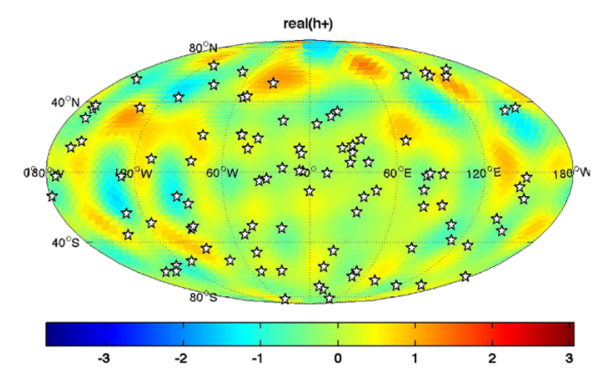

(d) Grad residual map (grad-recovered)

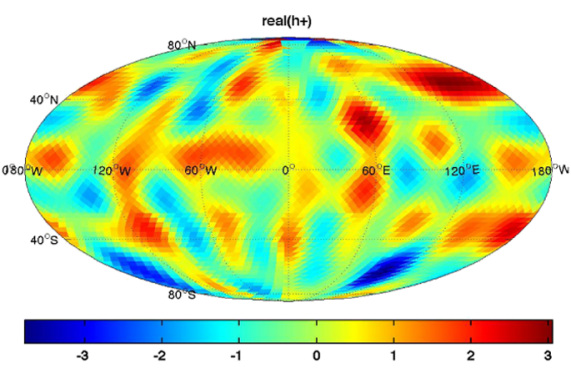

(b) Gradient component

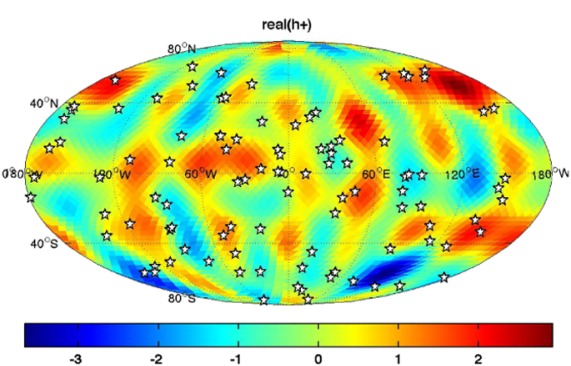

(e) Max-likelihood recovered map

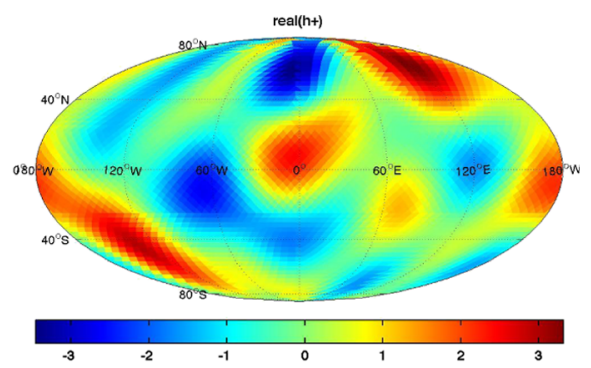

(c) Curl component

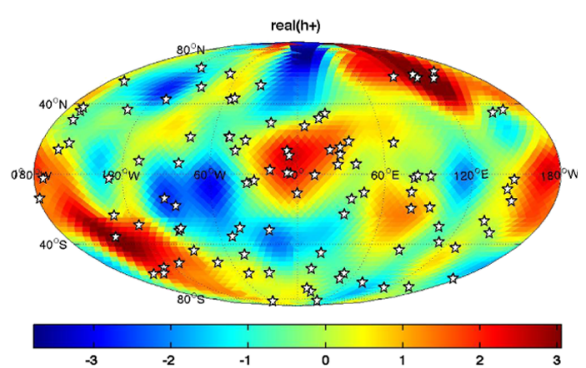

(f) Total residual map (total-recovered)

FIG. 11 (color online). Mollweide projections of $\operatorname{Re}\left(h_{+}\right)$for the different components of the simulated background (a)-(c), the maximum-likelihood recovered map for a PTA with $N=100$ pulsars (e), and the corresponding residual maps for the grad component (d) and the total simulated background (f). Sky maps of $\operatorname{Im}\left(h_{+}\right), \operatorname{Re}\left(h_{\times}\right)$, and $\operatorname{Im}\left(h_{\times}\right)$are similar. Note that the maximum-likelihood recovered map most closely resembles the gradient component of the simulated background, since a PTA is insensitive to the curl modes of a gravitational-wave background. The residual map for the grad component (d) is cleaner than the residual map for the total simulated background (f), which has angular structure that resembles the curl component of the background.

with a 100-pulsar array (see Sec. V D for details). It is apparent from the plots that the recovered maps more closely resemble the gradient component of the simulated background as the total number of pulsars in the array increases. The residual maps become "greener" (the values are getting closer to zero), and the angular scale of the structure in the residual maps generally gets smaller as the number of pulsars increases. But as discussed in Sec. IVA, we will never be able to recover the total simulated background, regardless of the number of pulsars in the array. This is because a PTA is insensitive to the curl component of the background. Figure 11 illustrates this point by comparing the maximum-likelihood recovered sky map for $N=100$ pulsars with the different components of the simulated background. The maximum-likelihood recovered map most closely resembles the gradient component of the simulated background, as expected.

\section{Implications of the limited sensitivity of a PTA}

A given PTA is only sensitive to a number of particular complex combinations of the $a_{(l m)}^{G}$ 's that is equal to the number of pulsars in the array. But which combinations are these? Considering the response functions, $R_{I(l m)}^{G}(f)=$ $(i 2 \pi f)^{-1} 2 \pi(-1)^{l} N_{l} Y_{(l m)}\left(\hat{u}_{I}\right)$, we see that for large $l$, $R_{I(l m)}^{G} \sim l^{-3 / 2}$. We therefore expect to have more sensitivity to low- $l$ modes. In an analysis with fewer modes than pulsars, and assuming that the noise matrix $F$ is a multiple of the identity, the Fisher information matrix is $H^{\dagger} H$, the elements of which are proportional to products of $R_{I(l \mathrm{~m})}^{G}$ 's. The square root of the diagonal elements of the inverse of the Fisher matrix provide a measure of the precision of parameter measurement, which indicates we would expect to measure the large- $l$ components of the background with a precision no better than $\sim l^{3 / 2}$. Considering the SVD of $H$ when there is a single pulsar in the array, we see that the column of $V$ corresponding to the nonzero singular value of $H$ is proportional to $R_{1(l m)}^{G}$, and this confirms again that we are most sensitive to the low- $l$ modes. We note that these are precisely the components you would get when decomposing the transverse-traceless projection of $\frac{1}{2} u_{1}^{a} u_{1}^{b} /(1+$ $\left.\hat{k} \cdot \hat{u}_{1}\right)$ onto the grad and curl spherical harmonic basis. Therefore, the gravitational-wave sky map corresponding to this single nonzero singular mode that we can measure is proportional to the response function for that single pulsar over the sky. This is to be expected — at fixed amplitude, an instrument is most sensitive to a signal distribution that matches its own relative sensitivity to different directions.

In practice, we can use this formalism to approach sky mapping in two different ways. First, we can acknowledge the fact that we are only sensitive to $N$ complex components of the background and limit the number of complex ( $\mathrm{lm}$ ) modes included in the model to be less than or equal 
to $N$. On average, we are equally sensitive to all $m$ modes corresponding to a particular $l$ (although the relative sensitivity to each $m$ mode will in practice depend on the pulsar distribution in the PTA). Therefore we should include complete sets of modes up to some $l_{\max }$, which means including $\left(l_{\max }+1\right)^{2}-4$ modes in total. [The $l$ th harmonic has $(2 l+1)$ independent $m$ modes, giving a total of $\left(l_{\max }+1\right)^{2}$ independent modes with $l \leq l_{\max }$, and we subtract 4 because there are no modes with $l=0$ or $l=1$.] Alternatively, we can include more than $N$ modes in the model, find the singular value decomposition of the $H$ matrix, and then recover the $N$ linear combinations of the $N_{m}$ modes to which the array is sensitive. As discussed in the preceding paragraph, we expect the measurements of the low- $l$ components to be more accurate than those of high-l components.

The next relevant question is how many pulsars do we need to produce a useful map of the gravitational-wave sky? It is reasonable to require the angular resolution of the map to be comparable to the scale on which individual gravitational-wave sources might be resolvable. In [63], it was shown that the angular resolution $\Delta \Omega$ of a PTA with 50 isotropically distributed pulsars to an individual gravitational-wave source scales as

$$
\Delta \Omega \propto 50\left(\frac{50}{N}\right)^{1 / 2}\left(\frac{10}{\mathrm{SNR}}\right)^{2} \mathrm{deg}^{2}
$$

This implies

$$
l \sim 25\left(\frac{\mathrm{SNR}}{10}\right)\left(\frac{N}{50}\right)^{\frac{1}{4}}
$$

where we have defined the angular resolution as $l \approx$ $180 / \sqrt{\Delta \Omega / \mathrm{deg}^{2}}$ to be consistent with the CMB literature. The maximum $l$ mode that can be probed with a set of $N$ pulsars scales as $l_{\max } \sim \sqrt{N+4}-1$, which grows faster than the single-source angular resolution. Assuming an SNR of $\sim 3$ is needed for detection, we find that we need $N \gtrsim 100$ pulsars to reach the angular scale of individual sources, with corresponding $l_{\max } \sim 10$ and angular resolution $\Delta \Omega \sim 400 \mathrm{deg}^{2}$. Although this is beyond the current capabilities of PTAs, the SKA could detect several thousand millisecond pulsars [38], some of which will be sufficiently stable to contribute usefully to PTA efforts. We may therefore not be able to reach this limit until the SKA era. However, we saw in Sec. III E that we only need to go to $l_{\max } \approx 4$ to recover an isotropic, unpolarized and uncorrelated background. To reach this resolution requires only 21 pulsars, which is fewer than ongoing PTA efforts are currently using. We should therefore be able to produce informative maps of the gravitational-wave background and constraints on the level of anisotropy in the near future.
The fact that we are sensitive only to a number of modes equal to the number of pulsars in the PTA is a consequence of the fact that the pulsar lines of sight are fixed, and so our detector does not scan across the sky. Other gravitationalwave detectors such as LIGO or LISA will scan the sky as Earth rotates or the spacecraft orbit the Sun, which will give these detectors sensitivity to all of the modes of the background, given sufficient integration time.

As described briefly in the introduction to this section, what we will be able to learn from measurements of the gravitational-wave background is influenced both by the resolution of the map and by the nature of the background. It is clear from Fig. 10 in the preceding section that adding pulsars to the array improves the resolution of the map, allowing us to resolve finer structures. As the number of pulsars is increased, the size of the structures that are resolved in the map and that are visible in the unresolved part of the background becomes smaller. We have argued that we need $\sim 21$ pulsars to reach $l_{\max } \approx 4$, which is required to confidently recover an isotropic, unpolarized and uncorrelated background, and we need $\sim 100$ pulsars to reach a resolution at which we could identify hot spots of the size expected for individual gravitational-wave sources. Once a map has been made, it can be used to constrain models. Even with a low resolution map, we can check if the coefficients are consistent with the expectation for any particular model. If the background is isotropic, unpolarized and uncorrelated, then with a map of any resolution we will be able to make a statement that the background is consistent with being isotropic, unpolarized and uncorrelated to a certain level. That level of consistency will improve as the resolution increases. If the background deviates from isotropic, unpolarized and uncorrelated, then we will most likely not be able to identify this with a very low resolution map, but at some critical point the departures will start to show significantly.

It is important to note that a typical model for the background will not predict precise values of the components of the background, $a_{(I m)}^{G}$, but their statistical properties, $\left\langle a_{(l m)}^{G} a_{\left(l^{\prime} m^{\prime}\right)}^{G^{\prime} *}\right\rangle=C_{l m l^{\prime} m^{\prime}}^{G G^{\prime}}$. Section V F below describes how to recast the detection problem as a measurement of the $C_{l m l^{\prime} m^{\prime}}^{G G^{\prime}}$ coefficients. A given background map is a representation of the specific values of the coefficients in our realization of the Universe and so even with perfect data (but finite resolution, i.e., a finite number of pulsars) the map will not be able to distinguish all possible models. This is the usual problem of cosmic variance familiar from other areas of astrophysics, including analysis of the CMB-we can observe only one Universe, which limits our ability to determine its statistical properties. An $N$-pulsar map can measure $N$ linear combinations of the (infinite set of) $a_{(l m)}^{G}$ 's, but to illustrate we will assume, for simplicity, that these are the $N$ coefficients of lowest $l$, and we will assume that we have measured them perfectly. A typical model can be represented by a specification of the correlation 
coefficients, written as a matrix $C$, and for an isotropic, unpolarized and uncorrelated background we have $C=I$. An alternative model will either predict a specific $C$ or, more likely, make some generic statement about its properties, e.g., the diagonal components are significantly different from each other or the off-diagonal components are significantly different from zero. We consider the first case, again for simplicity. The model selection problem would ask if the alternative model fits the measured data better than an isotropic background. Since in this simplified problem the two models have no free parameters, the Bayesian evidence is equal to the likelihood ratio and, denoting the measured parameters by $\vec{a}$, the difference in log evidence is just

$$
\Delta \ln \mathcal{Z}=-\frac{1}{2} \ln (\operatorname{det} C)-\frac{1}{2} \vec{a}^{\dagger}\left(C^{-1}-I\right) \vec{a} .
$$

If the difference in log evidence is large enough, we will disfavor the isotropic model. To decide if we will be able to distinguish two models with a given $N$-pulsar array, we can compute the expectation value, under the alternative model, of this difference in log evidence. This expectation value is

$$
\langle\Delta \ln \mathcal{Z}\rangle=\frac{1}{2}[\operatorname{Tr}(C)-N-\ln (\operatorname{det} C)]
$$

It would be possible to come up with a contrived model in which only one of the components of the correlation matrix differs from the identity. In such a scenario the addition of extra pulsars to the array does not improve the distinguishability of the models. This is not surprising, as in such a scenario only one or two of the coefficients have different statistical properties in the alternative model, so measuring additional coefficients by adding pulsars does not provide any discriminating power. In more realistic alternative models, many components of the correlation matrix will differ from the identity. In that case, the trace of $C$ will grow roughly like $N$ and so at some point the models will be distinguishable, with the number of pulsars required depending on the size of the differences in $C$. We will leave a complete investigation of map making, including the effects of imperfect measurement of the coefficients and marginalizing over unknown parameters in the model of the background, for future work, but this simple illustration serves to demonstrate how a map might be used to constrain models and how the ability to place constraints improves with the number of pulsars in the array. In practice, what we will do is reevaluate the map as new data and new pulsars are added to the array and look for signatures of anisotropy or correlation. As the maps contain all the measurable information about the background, anyone will be able to check predictions of their favorite model against the data without having to reanalyze the entire data set.

The insensitivity of a PTA to the curl modes of the background means, in principle, that we are losing half of the information that is available. However, most of the information about the physics generating the background should be encoded in both the grad and curl components. For an unpolarized and uncorrelated background, the components of the grad and curl modes are independent realizations from the same probability distribution, so either of them encodes the information about that probability distribution (which is the power distribution across the sky). For a point source, i.e., a binary, contributing to the background, the grad and curl components both encode the source parameters, but there are only a small number of parameters that characterize an individual system, and so these can be extracted from the grad components alone provided there is a sufficient number of pulsars in the array. Our ability to resolve the parameters of multiple individual sources will be limited by the number of pulsars in the array, but, as described above, we can only measure $N$ complex parameters at any given frequency using $N$ pulsars, and so this limitation applies whether we are sensitive to the curl modes or not. In principle, a correlated stochastic background generated in the early Universe could have a pure curl polarization, or a curl amplitude much greater than the grad amplitude. In that case we would never see such a background with a pulsar timing array and would clearly have incomplete information about the background. However, as described above, the distinction between grad and curl modes is origin dependent. If the background was completely dominated by the curl component, then the Solar System would be in a very special location in the Universe, and so this situation should be regarded as extremely unlikely on anthropic grounds. Being unable to measure the curl background does mean, however, that we will not be able to compare its statistical properties to those of the grad background, and so we will not be sensitive to statistical polarization of the background, i.e., that the statistical properties of the grad and curl components are significantly different (this will be discussed again in Sec. VI A). However, the same anthropic argument suggests that significant statistical polarization is unlikely. In summary, while the insensitivity of a PTA to a curl background means that we will not be able to measure all of its properties, the main physical properties of the background should be evident in the grad-only part of the background, and so PTA observations still have tremendous potential to inform us about gravitational physics.

\section{E. What combinations of timing residuals can an arbitrary background produce?}

The range of the matrix $H$ describes the only possible combinations of timing residuals that could be induced in a given array of pulsars by any gravitational-wave background. Cornish and van Haasteren found that this range is spanned by the eigenvectors of the Hellings and Downs correlation matrix (private communication). This follows straightforwardly from properties of the SVD. In the SVD, 
Eq. (128), the columns of $U$ are eigenvectors of the matrix $H H^{\dagger}$ and the nonzero elements of $\Sigma$ are the square roots of the nonzero eigenvalues of this matrix. Writing $\vec{r}=H \vec{a}$ we see that

$$
\vec{r} \vec{r}^{\dagger}=H \vec{a} \vec{a}^{\dagger} H^{\dagger}
$$

If we replace $\vec{a} \vec{a}^{\dagger}$ by the identity matrix, the right hand side of this equation becomes $\mathrm{HH}^{\dagger}$, which is the matrix we want. This replacement can be accomplished by appropriate averaging over the space of possible $\vec{a}$ 's, for instance by assuming these are uniformly distributed over equal ranges symmetric about zero or by assuming they have an isotropic Gaussian distribution with correlations $\left\langle a_{k} a_{k^{\prime}}^{*}\right\rangle=\delta_{k k^{\prime}}$. So far, we have used only generic properties of the SVD, but these observations are useful only if we can efficiently compute $\left\langle\vec{r} \vec{r}^{\dagger}\right\rangle$ for such a distribution of $\vec{a}$ 's. In our case the specification $\left\langle a_{k} a_{k^{\prime}}^{*}\right\rangle=\delta_{k k^{\prime}}$ describes an isotropic, unpolarized and uncorrelated background [see Eqs. (37)-(39)]. We know that the expected response for such a background is the Hellings and Downs correlation matrix and the result follows. That this is true in both our formulation and that of Cornish and van Haasteren is a consequence of the fact that an isotropic, unpolarized and uncorrelated background is described by $\left\langle a_{k} a_{k^{\prime}}^{*}\right\rangle=\delta_{k k^{\prime}}$ in both prescriptions. This argument effectively assumes that all possible modes are present in the vector $\vec{a}$, but a similar argument can be used if we have only included a subset $\mathcal{S}$ of these modes. The $U$ matrix is then different, but its columns will be the eigenvectors of $\left\langle\vec{r} \vec{r}^{\dagger}\right\rangle_{\mathcal{S}}$, which denotes the average response over an isotropic distribution of the modes included in $\mathcal{S}$ only. Using the results in this paper we can compute $\left\langle\vec{r} \vec{r}^{\dagger}\right\rangle_{\mathcal{S}}$ straightforwardly for arbitrary $\mathcal{S}$. Specifically, we use Eqs. (53) and (54) with $C_{l}=1$ and the sum over $l$ and $m$ replaced by a sum over the modes included in $\mathcal{S}$ and the response functions, $R_{I(l m)}^{P}(f)$, taken from Eq. (110). We saw in Sec. III E that including modes up to $l_{\max }=4$ was sufficient to recover the Hellings and Downs correlation curve, so we would expect that with these 21 modes in $\vec{a}$, the $U$ matrix would already be almost equal to the infinite coefficient limit.
In the case that all modes are present in the background, $V$ has infinitely many columns. However, once $U$ and $\Sigma$ have been found, it is possible to construct the first $N$ elements of the $j$ th column of $V^{\dagger}$ for arbitrary $j$ from

$$
\left(V^{\dagger}\right)_{i j}=\Sigma_{i K}^{+} U_{I K}^{*} R_{I j}^{G}
$$

where $R_{I j}^{G}$ is the grad response of pulsar $I$ to the component of the background included in the $j$ th element of $\vec{a}$. This follows by setting $a_{k}=0 \forall k \neq j$ and $a_{j}=1$ in $H \vec{a}$, which makes $(H \vec{a})_{I}=R_{I j}^{G}$. From the SVD we have

$$
H \vec{a}=U \Sigma \vec{V}_{j}^{\dagger}
$$

where $\vec{V}_{j}^{\dagger}$ denotes the $j$ th column of $V^{\dagger}$. Premultiplying by the inverse of $U$, which is $U^{\dagger}$, and then by the pseudoinverse of $\Sigma$ gives the final result. This picks out only the first $N$ elements of $\vec{V}_{j}^{\dagger}$ due to the zeros in the $\Sigma$ matrix. However, these first $N$ elements of $\vec{V}_{j}^{\dagger}$ are precisely the components of the background to which the PTA is sensitive, and this approach allows these to be computed to whatever resolution is required.

\section{F. Marginalized likelihood function for the $C_{l m l^{\prime} m^{\prime}}^{G G^{\prime}}$ 's}

We finish by noting that an alternative way to proceed would be to start with the above likelihood, but marginalize over the parameters $\vec{a}$. For simplicity, we assume in the following that we are using the real version of the likelihood (124) with twice as many real components in $\vec{a}$ and $H$. We will also assume a Gaussian distribution on $\vec{a}$ of the form

$$
p(\vec{a} \mid C)=\frac{1}{\sqrt{\operatorname{det}(2 \pi C)}} \exp \left\{-\frac{1}{2} \vec{a}^{T} C^{-1} \vec{a}\right\},
$$

where $C \equiv\left\{C_{l m l^{\prime} m^{\prime}}^{G G}\left(f_{k}, f_{k^{\prime}}\right)\right\}$ are the model hyperparameters defined by an equation like (106). Then by marginalizing over $\vec{a}$, we obtain the likelihood

$$
p(\delta t \mid F, C) \propto \frac{1}{\sqrt{\operatorname{det}\left(I+C H^{T} F^{-1} H\right)}} \exp \left\{-\frac{1}{2} \vec{\delta}^{T}\left(F^{-1}-F^{-1} H\left(C^{-1}+H^{T} F^{-1} H\right)^{-1} H^{T} F^{-1}\right) \vec{\delta} t\right\},
$$

where we have assumed $C^{T}=C$ and $F^{T}=F$. By using the Woodbury matrix lemma, this can be simplified to

$$
p(\delta t \mid F, C)=\frac{1}{\sqrt{\operatorname{det}(2 \pi D)}} \exp \left\{-\frac{1}{2} \vec{\delta}^{T} D^{-1} \vec{\delta} t\right\}, \quad \text { where } D=F+H C H^{T},
$$


which can be seen most readily by considering the correlation matrix directly rather than its inverse [64]. One can use this modified likelihood to construct posterior distributions or estimators of the parameters $C$ using Bayesian inference or frequentist statistics as before. In particular, one reasonable assumption to make in this case would be $C_{l m l^{\prime} m^{\prime}}^{G G}\left(f_{k}, f_{k^{\prime}}\right)=\delta_{k k^{\prime}} C_{l m l^{\prime} m^{\prime}}^{G G}$, i.e., that different frequency components are uncorrelated and the covariance of a given component depends only on the angular mode parameters, $l, m, l^{\prime}, m^{\prime}$, and not on the frequency. We note that this marginalization is completely analogous to the marginalization over timing-model parameters used to derive the timedomain version of Eq. (124) in [53]. If a flat prior is used instead of a Gaussian prior, the $C^{-1}$ term does not appear in the likelihood and the term inside the exponential can be simplified to the form $H_{\text {null }}\left(H_{\text {null }}^{T} F H_{\text {null }}\right)^{-1} H_{\text {null }}^{T}$, where $H_{\text {null }}$ is the matrix of left singular eigenvectors of $H$ with zero singular values. The matrix $H_{\text {null }}$ is given by the last $2 N_{m}-2 N$ columns of $U$ in the SVD (the factors of 2 come from going from a complex to a real representation). This is an identical result to the marginalization of the timing model parameters described in [53].

\section{DISCUSSION}

We have described how the formalism used to characterize the polarization of the CMB can be applied to the analysis of gravitational-wave backgrounds. Any symmetric transverse-traceless tensor field on the sphere can be decomposed into a superposition of modes that are gradients or curls of spherical harmonics. We considered using this decomposition to analyze a gravitational-wave background observed with a pulsar timing array. Writing a statistically isotropic background as a superposition of these modes, we found that the PTA overlap reduction functions for these modes were Legendre polynomials evaluated for the pulsar angular separations. We showed that this was just a consequence of a pulsar having zero response to curl modes, and a response to each gradient mode that is proportional to the corresponding spherical harmonic evaluated at the direction to the pulsar. Although an infinite number of modes are needed to precisely recover the Hellings and Downs curve for an isotropic, unpolarized and uncorrelated background, we showed that, in practice, the background can be recovered accurately using just the lowest three $l$ modes, $l=2,3,4$.

The formalism can also be applied to arbitrary backgrounds, and we have given general expressions for the overlap reduction functions of all possible modes. For PTAs, these expressions simplify considerably, being a sum of products of ordinary spherical harmonics, and being independent of any curl correlation coefficients. We have also shown how anisotropic, unpolarized and uncorrelated backgrounds can be represented in this formalism, and how the results derived here can be used to write down overlap reduction functions for arbitrary backgrounds of that type, extending the analytical results in [34] to orders above quadrupole. Finally, we described a formalism that can be used to reconstruct a map of the gravitational-wave sky for that part of the background, spanned by the gradient modes, that is visible to a PTA. A PTA containing $N$ pulsars measures two real-valued quantities (an amplitude and a phase or a sine and a cosine quadrature) at each frequency with each pulsar. With $2 N$ measurements we cannot hope to measure more than $2 N$ components of the background. These are $2 \mathrm{~N}$ combinations of the components of the gradient modes of the background, and we will never be able to detect curl modes of the background, no matter how many pulsars are included in the array. This insensitivity to curl modes arises as a consequence of the fact that the pulsar array is static and does not move or scan across the sky. To achieve the angular resolution expected for individually resolvable single sources, we need to probe modes of the background up to $l_{\max } \sim 10$, which will require about 100 pulsars. This should be achievable with the SKA.

For the standard case of an isotropic, unpolarized and uncorrelated background, the formalism described here is more complicated than directly using the Hellings and Downs curve to model the pulsar pair cross-correlations. However, it is only marginally more complicated; the individual overlap reduction functions are simpler, and the sensitivity to isotropic backgrounds should be comparable. The power of this new approach is that it provides a single unified framework to characterize any kind of background, free from any assumptions about its physical nature. We will obtain a map of the background that encodes as much information as is possible to extract about the background using the available data. It is no longer necessary to carry out separate searches for isotropic or anisotropic backgrounds, but the level of anisotropy can be determined by looking at the resulting map. It is not necessary to assume that the background is uncorrelated between different sky locations; instead the degree of correlation will be directly measured, which will provide sensitivity to possible new and unexpected physics. If the observed gravitational-wave background is found to show significant correlations between different points on the sky, it would be a very profound discovery. A gravitational-wave background generated by a superposition of astrophysical sources will not be correlated on the sky as the properties of each source, including the formation time and phase, will be independent from one another. While nonstandard scenarios of primordial background formation could in principle generate correlations in the background, in standard scenarios the nanohertz stochastic background is expected to be uncorrelated. An observation of a correlated nanohertz background would therefore be startling and necessitate serious theoretical work to develop a plausible mechanism to explain it. 
Although such an outcome is perhaps unlikely, the small additional cost of characterizing the background in this way seems very worthwhile, given the large potential reward if something unexpected is discovered.

\section{A. Implications of a correlated background}

We will now briefly consider the hypothetical implications of a correlated background for the pulsar timing residuals. To define the correlation between gravitational waves coming from two different points on the sky we need to relate the polarization axes at the two points, since these define the distinction between the plus and cross polarizations. A natural way to do this is to relate the axes by parallel transport. There is a unique geodesic (a great circle) linking any two points on the sky. Starting at the point with direction $\hat{k}$ for which the polarization axes are $\hat{l}, \hat{m}$ we can define new coordinates for the second point, $\hat{k}^{\prime}$, by taking $\hat{k}$ to be along the $z$ axis and $\hat{l}$ to be along the $x$ axis. If in this coordinate system $\hat{k}^{\prime}$ has spherical polar coordinates $\left(\theta^{\prime}, \phi^{\prime}\right)$, then the great circle connecting $\hat{k}$ to $\hat{k}^{\prime}$ is the constant azimuth line $\phi=\phi^{\prime}$. It can easily be seen that parallel transport of a vector $v^{a}$ on the sphere around a line of constant azimuth keeps both $v^{\theta}$ and $\sin \theta v^{\phi}$ constant. The natural polarization axes at $\hat{k}^{\prime}$ are therefore

$$
\begin{gathered}
\hat{l}^{\prime}=\left(\begin{array}{c}
\cos ^{2} \phi^{\prime} \cos \theta^{\prime}+\sin ^{2} \phi^{\prime} \\
\cos \phi^{\prime} \sin \phi^{\prime}\left(\cos \theta^{\prime}-1\right) \\
-\cos \phi^{\prime} \sin \theta^{\prime}
\end{array}\right), \\
\hat{m}^{\prime}=\left(\begin{array}{c}
\cos \phi^{\prime} \sin \phi^{\prime}\left(\cos \theta^{\prime}-1\right) \\
\sin ^{2} \phi^{\prime} \cos \theta^{\prime}+\cos ^{2} \phi^{\prime} \\
-\sin \phi^{\prime} \sin \theta^{\prime}
\end{array}\right) .
\end{gathered}
$$

In the CMB literature, the standard approach is to write down correlation functions for axes aligned along the line joining the two points, which is a great circle as above. It is easy to see that this is equivalent to the parallel transport approach. With this definition to relate the polarization axes and additionally making the specific choice that the $\hat{x}$ axis is aligned along the line joining the two points, it is possible to compute the expected correlation between the polarization states of the gravitational waves at different sky positions. Using Eq. (37) we find [36]

$$
\begin{aligned}
&\left\langle h_{+}(f, \hat{k}) h_{+}^{*}\left(f^{\prime}, \hat{k}^{\prime}\right)\right\rangle_{k}=\frac{1}{2} \sum_{l=2}^{\infty} \frac{2 l+1}{4 \pi}\left(N_{l}\right)^{2}\left[C_{l}^{G G}(f) G_{l 2}^{+}(\cos \theta)+C_{l}^{C C}(f) G_{l 2}^{-}(\cos \theta)\right] \delta\left(f-f^{\prime}\right), \\
&\left\langle h_{\times}(f, \hat{k}) h_{\times}^{*}\left(f^{\prime}, \hat{k}^{\prime}\right)\right\rangle_{k}=\frac{1}{2} \sum_{l=2}^{\infty} \frac{2 l+1}{4 \pi}\left(N_{l}\right)^{2}\left[C_{l}^{C C}(f) G_{l 2}^{+}(\cos \theta)+C_{l}^{G G}(f) G_{l 2}^{-}(\cos \theta)\right] \delta\left(f-f^{\prime}\right), \\
&\left\langle h_{+}(f, \hat{k}) h_{\times}^{*}\left(f^{\prime}, \hat{k}^{\prime}\right)\right\rangle_{k}=\frac{1}{2} \sum_{l=2}^{\infty} \frac{2 l+1}{4 \pi}\left(N_{l}\right)^{2}\left[C_{l}^{G C}(f) G_{l 2}^{+}(\cos \theta)-C_{l}^{C G}(f) G_{l 2}^{-}(\cos \theta)\right] \delta\left(f-f^{\prime}\right), \\
&\left\langle h_{\times}(f, \hat{k}) h_{+}^{*}\left(f^{\prime}, \hat{k}^{\prime}\right)\right\rangle_{k}=\frac{1}{2} \sum_{l=2}^{\infty} \frac{2 l+1}{4 \pi}\left(N_{l}\right)^{2}\left[C_{l}^{C G}(f) G_{l 2}^{+}(\cos \theta)-C_{l}^{G C}(f) G_{l 2}^{-}(\cos \theta)\right] \delta\left(f-f^{\prime}\right),
\end{aligned}
$$

where the subscript $k$ indicates we are defining the axes by parallel transport of the polarization axes at $\hat{k}$ to $\hat{k}^{\prime}$.

If we require the background to be statistically unpolarized, ${ }^{1}$ we must impose the constraint that the correlation functions are independent of the choice of polarization axes. Parallel transport is angle preserving, so if the axes at $\hat{k}$ are rotated by an angle $\psi$, the same is true for the parallelly transported axes at $\hat{k}^{\prime}$. Such a rotation transforms the plus and cross polarizations as

$$
\bar{h}_{+}=h_{+} \cos 2 \psi+h_{\times} \sin 2 \psi, \quad \bar{h}_{\times}=-h_{+} \sin 2 \psi+h_{\times} \cos 2 \psi,
$$

with the corresponding effect on the correlation function

$$
\begin{aligned}
\left\langle\bar{h}_{+}(f, \hat{k}) \bar{h}_{+}^{*}\left(f^{\prime}, \hat{k}^{\prime}\right)\right\rangle_{k}=\left\langle h_{+}(f, \hat{k}) h_{+}^{*}\left(f^{\prime}, \hat{k}^{\prime}\right)\right\rangle_{k} \cos ^{2} 2 \psi+\left\langle h_{\times}(f, \hat{k}) h_{\times}^{*}\left(f^{\prime}, \hat{k}^{\prime}\right)\right\rangle_{k} \sin ^{2} 2 \psi & \\
& +\left(\left\langle h_{+}(f, \hat{k}) h_{\times}^{*}\left(f^{\prime}, \hat{k}^{\prime}\right)\right\rangle_{k}+\left\langle h_{\times}(f, \hat{k}) h_{+}^{*}\left(f^{\prime}, \hat{k}^{\prime}\right)\right\rangle_{k}\right) \sin 2 \psi \cos 2 \psi .
\end{aligned}
$$

\footnotetext{
${ }^{1}$ By "statistically unpolarized" we mean that the expectation values $\left\langle h_{+}(f, \hat{k}) h_{+}^{*}\left(f^{\prime}, \hat{k}^{\prime}\right)\right\rangle_{k}$ and $\left\langle h_{\times}(f, \hat{k}) h_{\times}^{*}\left(f^{\prime}, \hat{k}^{\prime}\right)\right\rangle_{k}$ are equal. Any particular realization of the background will be polarized at each point (see Fig. 16), but that polarization will have no preferred direction when averaged over the sky.
} 
Requiring this to be unchanged we must have

$$
\begin{aligned}
\left\langle h_{+}(f, \hat{k}) h_{+}^{*}\left(f^{\prime}, \hat{k}^{\prime}\right)\right\rangle_{k} & =\left\langle h_{\times}(f, \hat{k}) h_{\times}^{*}\left(f^{\prime}, \hat{k}^{\prime}\right)\right\rangle_{k}, \\
\left\langle h_{+}(f, \hat{k}) h_{\times}^{*}\left(f^{\prime}, \hat{k}^{\prime}\right)\right\rangle_{k} & =-\left\langle h_{\times}(f, \hat{k}) h_{+}^{*}\left(f^{\prime}, \hat{k}^{\prime}\right)\right\rangle_{k},
\end{aligned}
$$

which imposes the restrictions $C_{l}^{G G}(f)=C_{l}^{C C}(f) \equiv C_{l}(f)$ and $C_{l}^{G C}(f)=-C_{l}^{C G}(f)$ for statistically unpolarized backgrounds. But since $C_{l}^{G C}(f)=0=C_{l}^{C G}(f)$ follows from invariance under parity transformations for a statistically isotropic background (as shown in Sec. II D), the above expectation values simplify further to

$$
\begin{aligned}
\left\langle h_{+}(f, \hat{k}) h_{+}^{*}\left(f^{\prime}, \hat{k}^{\prime}\right)\right\rangle_{k} & =\left\langle h_{\times}(f, \hat{k}) h_{\times}^{*}\left(f^{\prime}, \hat{k}^{\prime}\right)\right\rangle_{k} \\
& =\frac{1}{2} \sum_{l=2}^{\infty} \frac{2 l+1}{4 \pi}\left(N_{l}\right)^{2} C_{l}(f)\left[G_{l 2}^{+}(\cos \theta)+G_{l 2}^{-}(\cos \theta)\right] \delta\left(f-f^{\prime}\right), \\
\left\langle h_{+}(f, \hat{k}) h_{\times}^{*}\left(f^{\prime}, \hat{k}^{\prime}\right)\right\rangle_{k} & =\left\langle h_{\times}(f, \hat{k}) h_{+}^{*}\left(f^{\prime}, \hat{k}^{\prime}\right)\right\rangle_{k}=0 .
\end{aligned}
$$

We note that it is also possible to have a statistically polarized but isotropic background which would be characterized by $C_{l}^{G G}(f) \neq C_{l}^{C C}(f)$. The correlations computed from Eqs. (140) and (141) would then no longer be equal, but this is perfectly consistent as these correlation functions are referred to the special choice of axes with the $\hat{x}$ direction along the line joining the two pulsars. Since PTAs are insensitive to the curl modes of the background, we will not be able to identify statistically polarized backgrounds using pulsar timing arrays. As a final comment, we note that, whether statistically polarized or not, the individual modes in this decomposition describe a background that has nontrivial correlations in the emission from different sky locations, which would be a startling discovery as discussed above.

Another interesting feature of a correlated background is in the behavior of the pulsar term. As discussed in Sec. III A, for uncorrelated backgrounds in the limit $f L / c \gg 1$, the pulsar term averages to zero except for the pulsar autocorrelation, for which the pulsar term contributes an equal amount as the Earth term, increasing the total correlation by a factor of 2 . For a correlated background, by contrast, the pulsar term always averages to zero, even for the autocorrelation. Mathematically, this is because for each individual mode we evaluate the product of two integrals, and this reduces to the squared magnitude of the integral in the autocorrelation limit. The integral over the sky of the pulsar term gives zero in the limit $f L / c \rightarrow \infty$, so there is no contribution from this term to the final result. Physically, the factor of 2 in the case of an uncorrelated background arises from the fact that the correlation in the pulsar residuals is caused by the same gravitational waves being seen by both pulsars. When the pulsar is the same, the pulsar term in a given gravitational wave adds coherently, while they interfere destructively for different pulsars. In the correlated background case, the correlation is coming from the interaction between gravitational waves propagating in different directions, but with correlated phases. The pulsar terms for the same pulsar but from different sky positions do not add coherently because of the $(1+\hat{k} \cdot \hat{u})$ factor, and therefore there are no correlated contributions from the pulsar terms in that case.

\section{B. Distinguishing a correlated background-overlap reduction functions}

We have written a TEMPO2 plug-in to generate an arbitrary gravitational-wave background by prescribing the gradient and curl spherical harmonic coefficients, $\left\{a_{(l m)}^{G}, a_{(l m)}^{C}\right\}$. The plug-in populates the sky with a large number of gravitational-wave sources, whose complex amplitudes are chosen to be consistent with the specified coefficients. This can be used to generate both uncorrelated and correlated backgrounds. As a test of the plug-in, in Fig. 12 we show the average correlation (computed over 100 realizations of the background) for distinct pulsar pairs within a chosen ensemble of pulsars. The data sets assume an array of 32 pulsars spread uniformly over the sky and observed fortnightly for 5 years. The injected background power spectrum is flat, creating a correlated white-noise influence in pulsars separated across the sky. Results are shown for both an unpolarized, $l_{\max }=2$ correlated background and an isotropic, unpolarized and uncorrelated background. The expected analytic result is shown in both cases as well, normalized such that the correlation for pulsars with zero angular separation is equal to 1 . We see that the analytic results agree perfectly with the numerical calculations, and the scatter in the two cases is approximately the same.

The distinguishability of the two types of backgrounds can be assessed using Bayesian evidence. We took one of the correlated-background data set realizations used in Fig. 12 


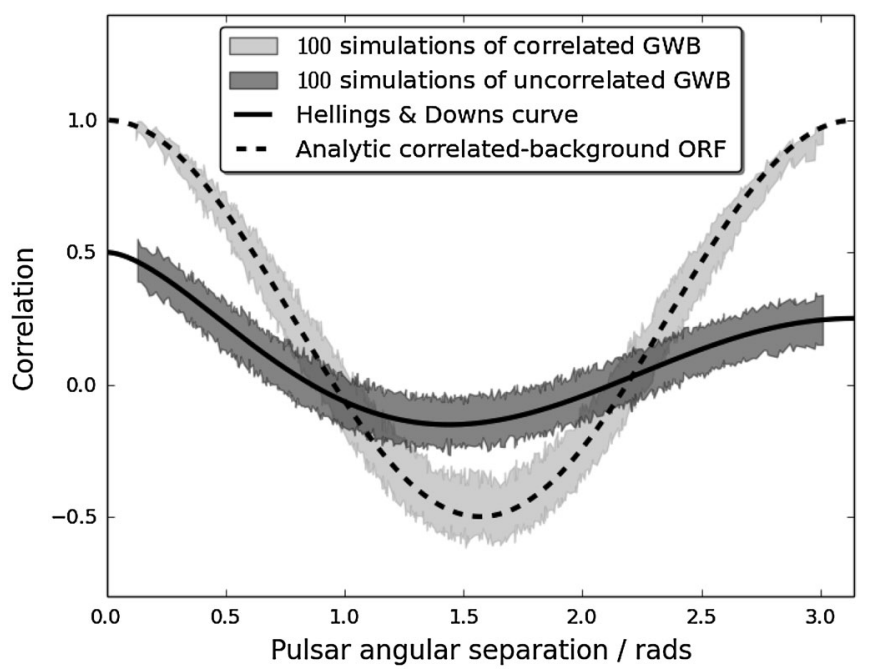

FIG. 12. The average correlation between pairs of pulsars (and errors), as a function of pulsar angular separation, for an unpolarized, $l_{\max }=2$ correlated gravitational-wave background (light-grey region) and for an isotropic, unpolarized and uncorrelated gravitational-wave background (dark-grey region). The averages were computed over 100 realizations of the backgrounds. Also shown are the analytic correlation functions expected in the two cases: the former being proportional to the Legendre polynomial $P_{2}(\cos \zeta)$, and the latter being the familiar Hellings and Downs curve.

(analyzing a subarray of 10 pulsars) and computed the Bayesian evidence within a time-domain likelihood formalism for models which assume that the background is correlated or uncorrelated. This gave a log evidence ratio of $\sim 500$ in favor of the correlated background, showing that the two types of background could be distinguished with high confidence. More work is needed to determine the threshold signal-to-noise ratio of the background that is needed to distinguish between the different models. The background considered here was very loud and would have been easily detectable regardless of the correlation signature.

Using this same data set, we then performed a modelindependent fully Bayesian reconstruction of the background-induced overlap reduction function. This technique was developed within the context of the first IPTA Data Challenge [65] to confirm that an isotropic distribution of uncorrelated astrophysical gravitational-wave sources will induce the distinctive Hellings and Downs signature in a cross-correlation analysis [66]. The overlap reduction function is parametrized at 13 distinct pulsar angular separations, and a cubic-spline interpolation used to compute the correlation at all other angular separations. The 13 "anchor" values of the overlap-reduction function are sampled, giving a posterior probability distribution that allows us to map out an envelope of all cubic splines which lie within desired credible intervals. Figure 13 shows this in practice, where the grey region defines the envelope of splines within the $95 \%$ credible interval of the full posterior

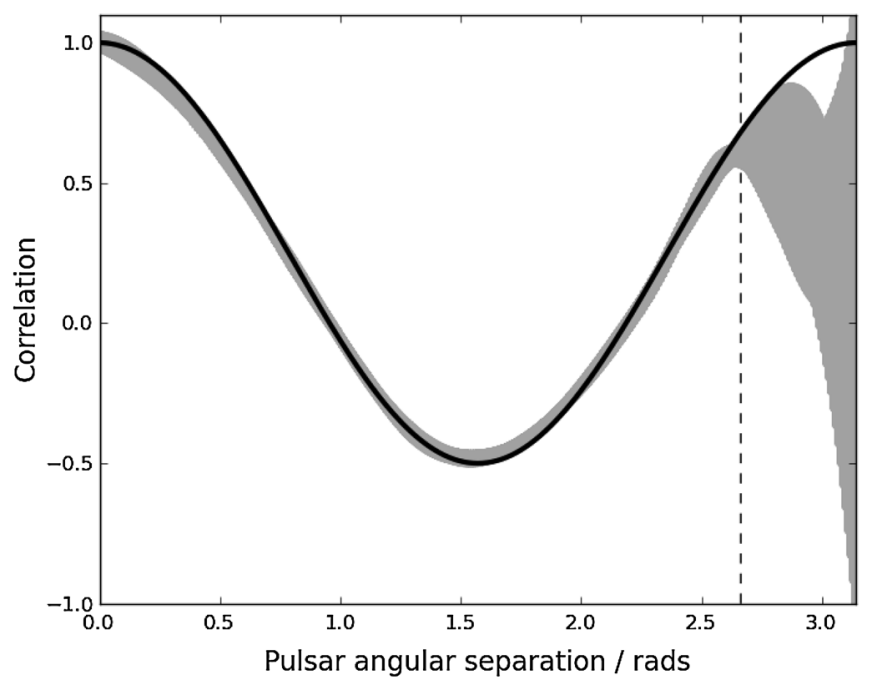

FIG. 13. A model-independent Bayesian reconstruction of the correlation between pairs of pulsars, as a function of pulsar angular separation, for a data set with an injected unpolarized, $l_{\max }=2$ correlated gravitational-wave background. The background spectrum is white, with the signal injected into 10 pulsars which are observed fortnightly over a time span of 5 years. The correlation is parametrized at 13 distinct separations, and a cubicspline interpolation used to compute the correlation at all other angular separations. The grey region shows the envelope of splines within the $95 \%$ credible interval of the full recovered posterior distribution, while the solid black curve is the expected analytic correlation function. The dashed black line indicates the largest angular separation between pulsars in our chosen array. As expected, beyond this line our reconstruction completely loses sensitivity.

probability distribution, the solid black line shows the expected overlap reduction function, and the dashed black line indicates the largest pulsar angular separation in this data set (beyond this we lose sensitivity). We see that not only can we distinguish between two alternative models, but we can directly measure the correlation function in the case that the background is correlated.

\section{Distinguishing a correlated background-sky maps}

Sky maps of $h_{+}$and $h_{\times}$will also allow us to distinguish between correlated and uncorrelated backgrounds, provided the angular resolution of the maps is finer than the angular scale of the correlated background. For coarser angular resolution, the correlations between neighboring sky directions would not be apparent in the maps, as the size of the pixels would be larger than the angular correlation scale of the background. Sky maps of gravitational-wave power, $P=\left|h_{+}\right|^{2}+\left|h_{\times}\right|^{2}$, lack some of the discriminating capability of sky maps of $h_{+}$and $h_{\times}$, since the phase relation between the different components of the waves is lost in the construction of the power map. 

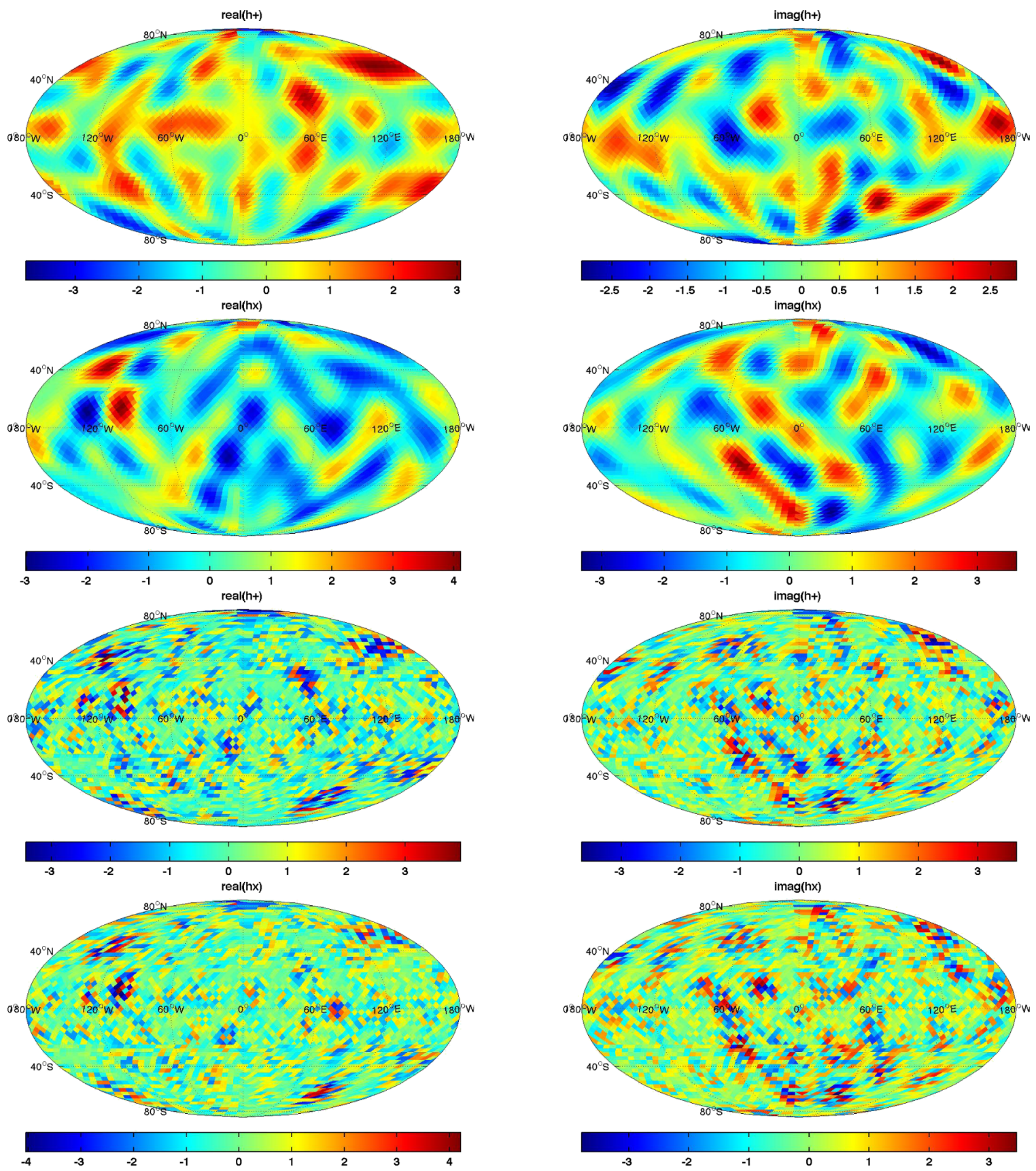

FIG. 14 (color online). Mollweide projections of the real and imaginary parts of $h_{+}$and $h_{\times}$for (a) a grad-only statistically isotropic correlated background with $C_{l}=1$ out to $l_{\max }=10$ (top four plots), and (b) an anisotropic uncorrelated background that has the same power distribution as the statistically isotropic background but uncorrelated phase (bottom four plots). Note the pixel-to-pixel variation in the maps for the uncorrelated background.

One problem that we encounter when making sky maps is that we measure only a single realization of the background, and thus do not have access to a statistical ensemble of such backgrounds. This means that even if the background is statistically isotropic, any particular realization will be anisotropic, having random hot and cold patches at different locations on the sky. Although one can calculate a set of $C_{l}$ 's for a correlated background that is consistent with the observed power on the sky (as they do for the $\mathrm{CMB}$ ), one could equally well regard the power distribution as fundamental and decompose it in terms of ordinary (i.e., scalar) spherical harmonics, effectively assuming it to be an anisotropic uncorrelated background, as in [34]. Although the latter approach is a way to describe any power distribution on the sky, the approach we adopt in this paper is more generic since it also incorporates the phase information of the waves at different points on the sky. 


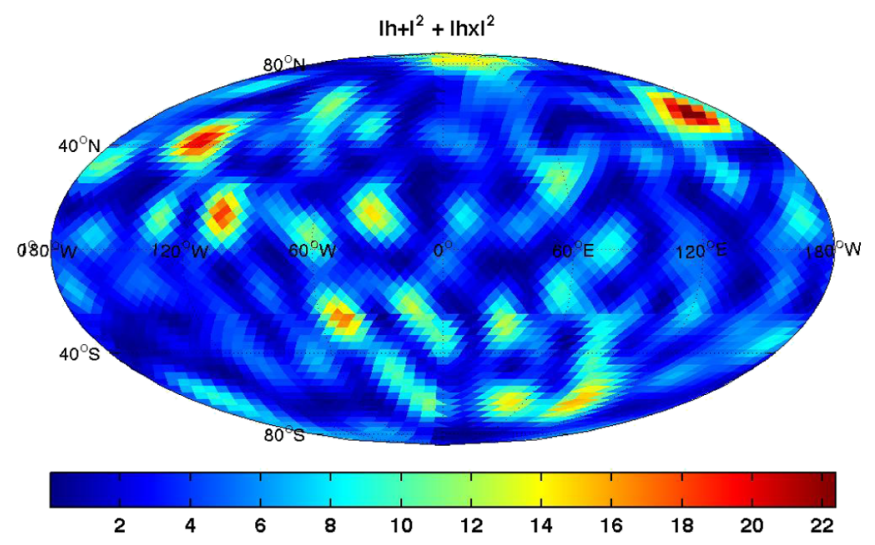

FIG. 15 (color online). Mollweide projection of the gravitational-wave power on the sky, $\left|h_{+}\right|^{2}+\left|h_{\times}\right|^{2}$, for both the statistically isotropic correlated background and the anisotropic uncorrelated background shown in Fig. 14.

To illustrate these statements, we simulated two gravitational-wave backgrounds that have exactly the same power distribution but different correlation properties: (a) a grad-only statistically isotropic correlated background with angular power $C_{l}=1$ for $l=2,3, \ldots, l_{\max }$, where $l_{\max }=10$, and (b) an anisotropic uncorrelated background whose power distribution is the same as that of the statistically isotropic background but has uncorrelated phase. (Both of these simulated backgrounds are statistically unpolarized.) The statistically isotropic background is the same as the gradient component of the simulated background that we analyzed in Sec. V C. (We consider only the gradient component for this discussion, since PTAs are insensitive to the curl component.) The anisotropic uncorrelated background is constructed from the statistically isotropic background by randomly rotating the polarization angle of the waves at each pixel on the sky. Maps of the real and imaginary parts of $h_{+}$and $h_{\times}$for both types of backgrounds are shown in Fig. 14. A sky map of the gravitational-wave power, $P=\left|h_{+}\right|^{2}+\left|h_{\times}\right|^{2}$, which is the same for both backgrounds, is shown in Fig. 15.

The size of the pixels in these sky maps is the same as that used for the maps in Secs. III A and V C, having an angular resolution $\Delta \Omega \sim 50 \mathrm{deg}^{2}$. This angular resolution corresponds to $l \sim 25$, which is substantially larger than the $l_{\max }(=10)$ used for the simulated statistically isotropic correlated background. To actually resolve individual pixels of this size using timing residual measurements would require a PTA with $N \gtrsim 700$ pulsars, which is the number of modes corresponding to $l_{\max } \sim 25$. This particular example is thus relevant for a PTA from the advanced SKA era. But the same qualitative conclusions that we will make here can also be made for a less ambitious PTA having $N \gtrsim 100$ pulsars and $l \sim 10$, provided $l_{\max }$ for the correlated background is sufficiently small (e.g., $l_{\max } \lesssim 5$ ) in order to ensure that the angular scale over which the phase is correlated is resolved by the measurements.

The additional information contained in the phase of $h_{+}$ and $h_{\times}$at each pixel can also be represented graphically by plotting polarization ellipses for the two different backgrounds, as shown in Fig. 16. The polarization ellipses are constructed as follows, using an approach similar to that for electromagnetic waves; see, e.g., Sec. 7.2 of [67].

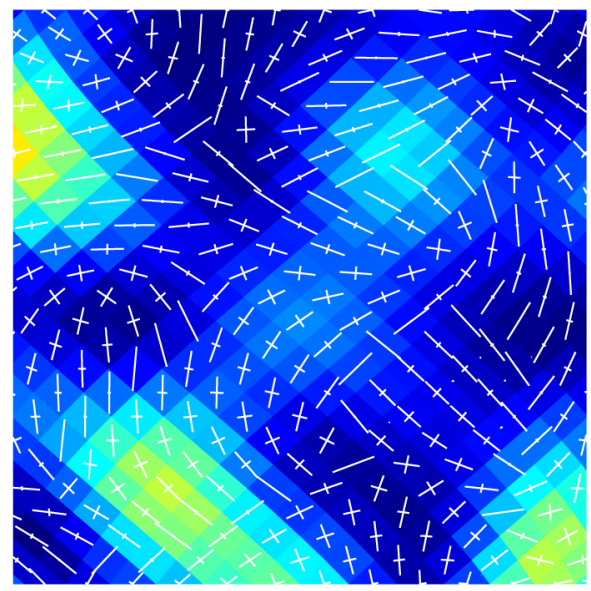

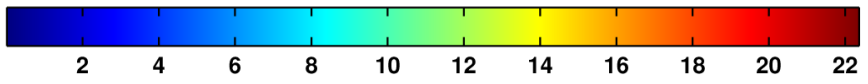

(a) Statistically isotropic background

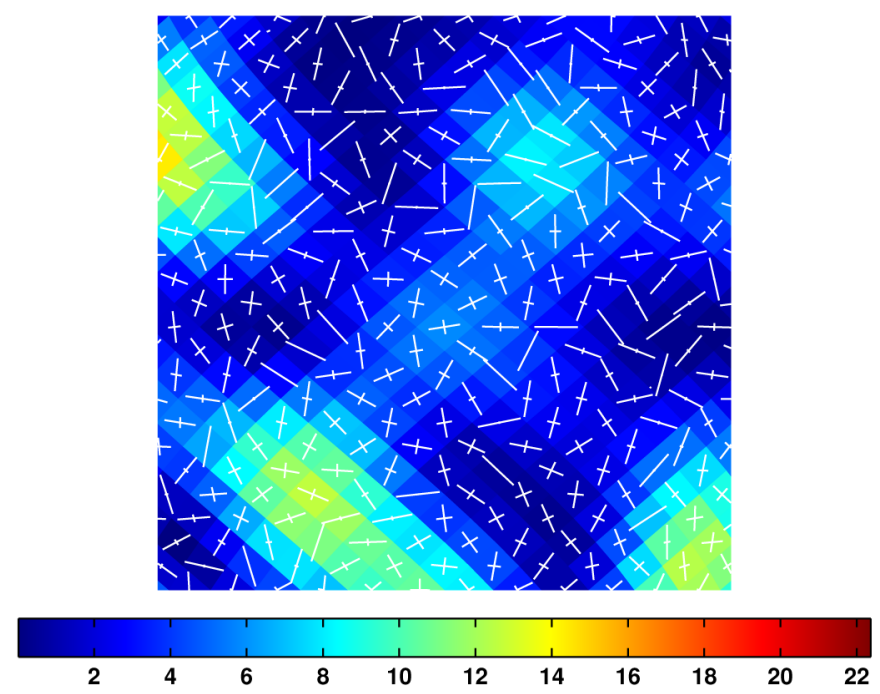

(b) Anisotropic uncorrelated background

FIG. 16 (color online). Polarization ellipses for a patch of sky (centered at $0^{\circ}$ latitude and $0^{\circ}$ longitude) for (a) the statistically isotropic correlated background, and (b) the anisotropic uncorrelated background shown in Fig. 14. The polarization ellipses are superimposed on the (common) map of gravitational-wave power shown in Fig. 15. The crosses indicate the direction and principal axes of the $h_{+}, h_{\times}$ polarization ellipses. Linear polarization in a particular direction is represented by a line, and circular polarization by a cross with equallength axes. Note that the polarization ellipses vary smoothly over the sky for the statistically isotropic correlated background, as compared to that for the anisotropic uncorrelated background, which have randomly oriented phase angles. 
Given the complex values of $h_{+}$and $h_{\times}$at each pixel for the two backgrounds, we first calculate the left- and rightcircularly polarized combinations $h_{L, R} \equiv\left(h_{+} \pm i h_{\times}\right) / \sqrt{2}$. If $\left|h_{L} / h_{R}\right|<1$, the polarization ellipse has semimajor and semiminor axes $a=1+r, b=1-r$, where $r=\left|h_{L} / h_{R}\right|$, and it is rotated by an angle $\psi \equiv \alpha / 2$ with respect to the horizontal (i.e., $\theta=$ const), where $\alpha=$ phase $\left(h_{L} / h_{R}\right)$. If $\left|h_{L} / h_{R}\right|>1$, we need to switch $h_{L}$ and $h_{R}$ in the above expressions for $r$ and $\alpha$. The angle $\psi$ is the polarization angle. Circular and linear polarization are special cases: $r=0$ corresponds to circular polarization, represented by a cross with equal-length axes; $r=1$ corresponds to linear polarization, represented by a line in a particular direction. Note that the polarization ellipses vary smoothly over the sky for the statistically isotropic correlated background, as compared to that for the anisotropic uncorrelated background. We therefore see that sky maps of $h_{+}$and $h_{\times}$have the potential to yield much more information than a map of just the total gravitational-wave power.

\section{Outlook}

The framework described here provides a completely generic approach to mapping the gravitational-wave sky using pulsar timing arrays. Our results suggest that it can be used to map the expected uncorrelated gravitational-wave background with almost as much sensitivity as the standard approach, while providing sensitivity to new physics. Further work is required to fully assess the computational costs and sensitivity of the approach, but we expect this will be a useful framework for future analyses. While we have focused on pulsar timing arrays in this work, the same approach can also be used to characterize gravitationalwave backgrounds in other frequency bands, relevant to ground-based or space-based detectors. We gave the overlap reduction function for a static interferometer in Appendix D. The extension to more realistic moving detectors is more involved, but work in this direction is underway.

\section{ACKNOWLEDGMENTS}

J. G.'s work is supported by the Royal Society. J. D. R. acknowledges support from NSF Grants No. PHY-1205585 and CREST No. HRD-1242090. S. R. T. acknowledges the support of the STFC and the RAS. C. M. F. M. acknowledges the support of the RAS, Universitas 21 and a Marie Curie International Outgoing Fellowship within the 7th European Community Framework Programme. We thank Sanjit Mitra for initial discussions, and Bruce Allen and Chiara Caprini for many e-mail exchanges regarding models for correlated backgrounds. C. M. F. M. thanks Gary Hinshaw for pointing her to Ref. [40], and J. D. R. thanks Graham Woan for useful feedback regarding an earlier draft of the paper. The authors also thank the anonymous referee for useful comments on the manuscript.
This research has made use of Python and its standard libraries: numpy and matplotlib. We have also made use of MEALPix (a Matlab implementation of HEALPix [68]), developed by the GWAstro Research Group and available from http://gwastro.psu.edu. This work was performed using the Darwin Supercomputer of the University of Cambridge High Performance Computing Service (http:// www.hpc.cam.ac.uk/), provided by Dell Inc. using Strategic Research Infrastructure Funding from the Higher Education Funding Council for England and funding from the Science and Technology Facilities Council.

\section{APPENDIX A: SPIN-WEIGHTED SPHERICAL HARMONICS}

The following is a list of some useful relations involving spin-weighted and ordinary spherical harmonics, ${ }_{s} Y_{l m}(\hat{k})$ and $Y_{l m}(\hat{k})$. For more details, see, e.g., [44] and [69]. Note that we use a slightly different normalization convention than in [44]. Namely, we put the Condon-Shortley factor $(-1)^{m}$ in the definition of the associated Legendre functions $P_{l}^{m}(x)$, and thus do not explicitly include it in the definition of the spherical harmonics. Also, for our analysis, we can restrict attention to spin-weighted spherical harmonics having integral spin weight $s$, even though spinweighted spherical harmonics with half-integral spin weight exist.

Ordinary spherical harmonics:

$$
\begin{aligned}
& Y_{l m}(\hat{k})=Y_{l m}(\theta, \phi)=N_{l}^{m} P_{l}^{m}(\cos \theta) e^{i m \phi}, \\
& N_{l}^{m}=\sqrt{\frac{2 l+1}{4 \pi} \frac{(l-m) !}{(l+m) !}}
\end{aligned}
$$

Relation of spin-weighted spherical harmonics to ordinary spherical harmonics:

$$
\begin{aligned}
& { }_{s} Y_{l m}(\theta, \phi)=\sqrt{\frac{(l-s) !}{(l+s) !}} \partial^{s} Y_{l m}(\theta, \phi) \text { for } 0 \leq s \leq l, \\
& { }_{s} Y_{l m}(\theta, \phi)=\sqrt{\frac{(l+s) !}{(l-s) !}}(-1)^{s} \bar{\partial}^{-s} Y_{l m}(\theta, \phi) \text { for }-l \leq s \leq 0,
\end{aligned}
$$

where

$$
\begin{aligned}
& ð \eta=-(\sin \theta)^{s}\left[\frac{\partial}{\partial \theta}+i \csc \theta \frac{\partial}{\partial \phi}\right](\sin \theta)^{-s} \eta, \\
& \bar{\jmath} \eta=-(\sin \theta)^{-s}\left[\frac{\partial}{\partial \theta}-i \csc \theta \frac{\partial}{\partial \phi}\right](\sin \theta)^{s} \eta,
\end{aligned}
$$

and $\eta=\eta(\theta, \phi)$ is a spin-s scalar field. 
Series representation:

$$
{ }_{s} Y_{l m}(\theta, \phi)=(-1)^{m}\left[\frac{(l+m) !(l-m) ! 2 l+1}{(l+s) !(l-s) !} \frac{1 / 2}{4 \pi}\right]^{1 / 2}(\sin \theta / 2)^{2 l} \sum_{k=0}^{l-s}\left(\begin{array}{c}
l-s \\
k
\end{array}\right)\left(\begin{array}{c}
l+s \\
k+s-m
\end{array}\right)(-1)^{l-k-s} e^{i m \phi}(\cot \theta / 2)^{2 k+s-m} .
$$

Complex conjugate:

$$
{ }_{s} Y_{l m}^{*}(\theta, \phi)=(-1)^{m+s} Y_{l,-m}(\theta, \phi) .
$$

Relation to Wigner rotation matrices:

$$
\begin{aligned}
D_{m^{\prime} m}^{l}(\phi, \theta, \psi) & =(-1)^{m^{\prime}} \sqrt{\frac{4 \pi}{2 l+1}}{ }^{m} Y_{l,-m^{\prime}}(\theta, \phi) e^{-i m \psi}, \\
{\left[D_{m^{\prime} m}^{l}(\phi, \theta, \psi)\right]^{*} } & =(-1)^{m} \sqrt{\frac{4 \pi}{2 l+1}}-m Y_{l, m^{\prime}}(\theta, \phi) e^{i m \psi} .
\end{aligned}
$$

Parity transformation:

$$
{ }_{s} Y_{l m}(\pi-\theta, \phi+\pi)=(-1)_{-s}^{l} Y_{l m}(\theta, \phi) .
$$

Orthonormality (for fixed $s$ ):

$$
\begin{aligned}
& \int_{S^{2}} \mathrm{~d}^{2} \Omega_{\hat{k} s} Y_{l m}(\hat{k})_{s} Y_{l^{\prime} m^{\prime}}^{*}(\hat{k}) \\
& \quad \equiv \int_{0}^{2 \pi} \mathrm{d} \phi \int_{0}^{\pi} \sin \theta \mathrm{d} \theta_{s} Y_{l m}(\theta, \phi)_{s} Y_{l^{\prime} m^{\prime}}^{*}(\theta, \phi) \\
& =\delta_{l l^{\prime}} \delta_{m m^{\prime}} .
\end{aligned}
$$

Addition theorem for spin-weighted spherical harmonics:

$$
\begin{aligned}
& \sum_{m=-l}^{l}{ }_{s} Y_{l m}\left(\theta_{1}, \phi_{1}\right)_{s^{\prime}} Y_{l m}^{*}\left(\theta_{2}, \phi_{2}\right) \\
& \quad=(-1)^{-s^{\prime}} \sqrt{\frac{2 l+1}{4 \pi}}-s^{\prime} Y_{l s}\left(\theta_{3}, \phi_{3}\right) e^{i s^{\prime} \chi_{3}},
\end{aligned}
$$

where

$$
\cos \theta_{3}=\cos \theta_{1} \cos \theta_{2}+\sin \theta_{1} \sin \theta_{2} \cos \left(\phi_{2}-\phi_{1}\right)
$$

and

$$
\begin{aligned}
e^{-i\left(\phi_{3}+\chi_{3}\right) / 2} & =\frac{\cos \frac{1}{2}\left(\phi_{2}-\phi_{1}\right) \cos \frac{1}{2}\left(\theta_{2}-\theta_{1}\right)-i \sin \frac{1}{2}\left(\phi_{2}-\phi_{1}\right) \cos \frac{1}{2}\left(\theta_{1}+\theta_{2}\right)}{\sqrt{\cos ^{2} \frac{1}{2}\left(\phi_{2}-\phi_{1}\right) \cos ^{2} \frac{1}{2}\left(\theta_{2}-\theta_{1}\right)+\sin ^{2} \frac{1}{2}\left(\phi_{2}-\phi_{1}\right) \cos ^{2} \frac{1}{2}\left(\theta_{1}+\theta_{2}\right)}}, \\
e^{i\left(\phi_{3}-\chi_{3}\right) / 2} & =\frac{\cos \frac{1}{2}\left(\phi_{2}-\phi_{1}\right) \sin \frac{1}{2}\left(\theta_{2}-\theta_{1}\right)+i \sin \frac{1}{2}\left(\phi_{2}-\phi_{1}\right) \sin \frac{1}{2}\left(\theta_{1}+\theta_{2}\right)}{\sqrt{\cos ^{2} \frac{1}{2}\left(\phi_{2}-\phi_{1}\right) \sin ^{2} \frac{1}{2}\left(\theta_{2}-\theta_{1}\right)+\sin ^{2} \frac{1}{2}\left(\phi_{2}-\phi_{1}\right) \sin ^{2} \frac{1}{2}\left(\theta_{1}+\theta_{2}\right)}} .
\end{aligned}
$$

Addition theorem for ordinary spherical harmonics:

$$
\sum_{m=-l}^{l} Y_{l m}\left(\hat{k}_{1}\right) Y_{l m}^{*}\left(\hat{k}_{2}\right)=\frac{2 l+1}{4 \pi} P_{l}\left(\hat{k}_{1} \cdot \hat{k}_{2}\right) .
$$

Integral of a product of spin-weighted spherical harmonics:

$$
\int_{S^{2}} \mathrm{~d}^{2} \Omega_{\hat{k} s_{1}} Y_{l_{1} m_{1}}(\hat{k})_{s_{2}} Y_{l_{2} m_{3}}(\hat{k})_{s_{3}} Y_{l_{3} m_{3}}(\hat{k})=\sqrt{\frac{\left(2 l_{1}+1\right)\left(2 l_{2}+1\right)\left(2 l_{3}+1\right)}{4 \pi}}\left(\begin{array}{ccc}
l_{1} & l_{2} & l_{3} \\
m_{1} & m_{2} & m_{3}
\end{array}\right)\left(\begin{array}{ccc}
l_{1} & l_{2} & l_{3} \\
-s_{1} & -s_{2} & -s_{3}
\end{array}\right) \text {, }
$$

where $\left(\begin{array}{ccc}l_{1} & l_{2} & l_{3} \\ m_{1} & m_{2} & m_{3}\end{array}\right)$ is a Wigner $3-j$ symbol. It can be written as 


$$
\begin{aligned}
\left(\begin{array}{ccc}
l & l^{\prime} & L \\
m & m^{\prime} & M
\end{array}\right)= & \sqrt{\frac{\left(l+l^{\prime}-L\right) !\left(l-l^{\prime}+L\right) !\left(-l+l^{\prime}+L\right) !(l+m) !(l-m) !\left(l^{\prime}+m^{\prime}\right) !\left(l^{\prime}-m^{\prime}\right) !(L+M) !(L-M) !}{\left(l+l^{\prime}+L+1\right) !}} \\
& \times \sum_{z \in \mathbb{Z}} \frac{(-1)^{z+l+l^{\prime}-M}}{z !\left(l+l^{\prime}-L-z\right) !(l-m-z) !\left(l^{\prime}+m^{\prime}-z\right) !\left(L-l^{\prime}+m+z\right) !\left(L-l-m^{\prime}+z\right) !}
\end{aligned}
$$

See, e.g., $[58,59,70]$, and references therein. Note that although this sum is over all integers, it contains only a finite number of nonzero terms since the factorial of a negative number is defined to be infinite.

\section{APPENDIX B: LEGENDRE POLYNOMIALS AND ASSOCIATED LEGENDRE FUNCTIONS}

The following is a list of some useful relations involving Legendre polynomials $P_{l}(x)$ and associated Legendre functions $P_{l}^{m}(x)$. For additional properties, see, e.g., [71].

Differential equation:

$$
\begin{aligned}
& \left(1-x^{2}\right) \frac{\mathrm{d}^{2}}{\mathrm{~d} x^{2}} P_{l}^{m}(x)-2 x \frac{\mathrm{d}}{\mathrm{d} x} P_{l}^{m}(x) \\
& +\left[l(l+1)-\frac{m^{2}}{\left(1-x^{2}\right)}\right] P_{l}^{m}(x)=0 .
\end{aligned}
$$

A useful recurrence relation:

$$
\left(x^{2}-1\right) \frac{\mathrm{d}}{\mathrm{d} x} P_{l}^{m}(x)=l x P_{l}^{m}(x)-(l+m) P_{l-1}^{m}(x) .
$$

Relation to ordinary Legendre polynomials, for $m=0,1, \ldots, l$ :

$$
\begin{aligned}
P_{l}^{m}(x) & =(-1)^{m}\left(1-x^{2}\right)^{m / 2} \frac{\mathrm{d}^{m}}{\mathrm{~d} x^{m}} P_{l}(x), \\
P_{l}^{-m}(x) & =(-1)^{m} \frac{(l-m) !}{(l+m) !} P_{l}^{m}(x) .
\end{aligned}
$$

Rodrigues' formula for $P_{l}(x)$ :

$$
P_{l}(x)=\frac{1}{2^{l} l !} \frac{\mathrm{d}^{l}}{\mathrm{~d} x^{l}}\left[\left(x^{2}-1\right)^{l}\right] .
$$

Series representation of Legendre polynomials:

$$
\begin{aligned}
P_{l}(x) & =\sum_{k=0}^{l}(-1)^{k} \frac{(l+k) !}{(k !)^{2}(l-k) !}\left(\frac{1-x}{2}\right)^{k} \\
& =\sum_{k=0}^{l}(-1)^{l+k} \frac{(l+k) !}{(k !)^{2}(l-k) !}\left(\frac{1+x}{2}\right)^{k} .
\end{aligned}
$$

Orthogonality relation (for fixed $m$ ):

$$
\int_{-1}^{1} \mathrm{~d} x P_{l}^{m}(x) P_{l^{\prime}}^{m}(x)=\frac{2(l+m) !}{(2 l+1)(l-m) !} \delta_{l l^{\prime}} .
$$

Shifted Legendre polynomials (defined for $x \in[0,1]$ ):

$$
\tilde{P}_{l}(x) \equiv P_{l}(1-2 x)=\frac{1}{l !} \frac{\mathrm{d}^{l}}{\mathrm{~d} x^{l}}\left(\left(x-x^{2}\right)^{l}\right) .
$$

\section{APPENDIX C: OSCILLATORY BEHAVIOR OF THE PULSAR TERM}

In Sec. III A, we assumed that the contribution of the oscillatory term $-2 \cos [2 \pi f L(1+\hat{k} \cdot \hat{u}) / c]$ to the integral for the autocorrelation was small, as it would be suppressed by at least a factor of $\sim 1 /(f L / c)$. Here we calculate the exact expression for the contribution from this term for an isotropic, unpolarized and uncorrelated gravitational-wave background. Analogous calculations may be carried out for higher multipole moments.

If we include the pulsar term, the autocorrelation function for an isotropic, unpolarized and uncorrelated background is given by

$$
\Gamma_{\text {auto }}(f)=\frac{1}{(2 \pi f)^{2}} \int_{S^{2}} \mathrm{~d}^{2} \Omega_{\hat{k}}(2-2 \cos [2 \pi f L(1+\hat{k} \cdot \hat{u}) / c])\left[\left|F^{+}(\hat{k})\right|^{2}+\left|F^{\times}(\hat{k})\right|^{2}\right],
$$

where $F^{+, \times}(\hat{k})$ are given in Eq. (61). [This is the same expression as in [34], but with an additional overall factor of $1 /(2 \pi f)^{2}$, which comes from the relation between the timing residual and redshift response functions.] If we choose coordinates so that the pulsar is located along the $z$ axis, then

$$
F^{+}(\hat{k})=\frac{1}{2}(1-\cos \theta), \quad F^{\times}(\hat{k})=0
$$

for which 


$$
\begin{aligned}
\Gamma_{\text {auto }}(f)=\frac{1}{(2 \pi f)^{2}} \int_{S^{2}} \mathrm{~d}^{2} \Omega_{\hat{k}}(2-2 \cos [2 \pi f L(1+\cos \theta) / c]) \\
\times \frac{1}{4}(1-\cos \theta)^{2} \\
=\frac{\pi}{(2 \pi f)^{2}} \int_{-1}^{1} \mathrm{~d} x(1-\cos [2 \pi f L(1+x) / c])(1-x)^{2} .
\end{aligned}
$$

This last integral is fairly simple to evaluate, the result being

$$
\Gamma_{\text {auto }}(f)=\frac{1}{(2 \pi f)^{2}}\left\{\frac{8 \pi}{3}-\frac{1}{\pi(f L / c)^{2}}\left[1-j_{0}(4 \pi f L / c)\right]\right\},
$$

where $j_{0}(x) \equiv \sin x / x$ is a spherical Bessel function of the first kind. Thus, for an isotropic, unpolarized and uncorrelated background, the oscillatory term is actually suppressed by a factor of $1 /(f L / c)^{2}$. The pulsar-term contribution to the autocorrelation is therefore well approximated by multiplying the overlap reduction function by a factor of 2 and neglecting the oscillatory term. For a correlated gravitational-wave background, there is no factor of 2 contribution from the pulsar term, as discussed in Sec. VI.

\section{APPENDIX D: RESPONSE OF A STATIC INTERFEROMETER}

In the point-detector limit the strain response of a static interferometer whose vertex is located at the origin of coordinates may be approximated by

$$
R^{A}(f, k)=\frac{1}{2} e_{a b}^{A}(\hat{k})\left(u_{1}^{a} u_{1}^{b}-u_{2}^{a} u_{2}^{b}\right),
$$

where $\hat{u}_{1}$ and $\hat{u}_{2}$ are unit vectors pointing along the two arms of the detector. We can evaluate this response in a computational frame in which $\hat{u}_{1}$ is in the $\hat{z}$ direction and $\hat{u}_{2}$ is in the $\hat{x}$ direction. We consider the $\hat{u}_{1}$ term of the response only to start with, which we denote by $R_{1(l m)}^{P}(f)$. In the computational frame $e_{a b}^{+}(\hat{k}) u_{1}^{a} u_{1}^{b}=\sin ^{2} \theta$ and $e_{a b}^{\times}(\hat{k}) u_{1}^{a} u_{1}^{b}=0$. This is independent of $\phi$, so as before we need $m=0$, and since $X_{(l 0)}(\hat{k})=0$, we find $R_{1(l m)}^{C}=0$ for all $l, m$. We also have $R_{1(l m)}^{G}=0$ for $m \neq 0$ and

$$
\begin{aligned}
R_{1(l 0)}^{G}(f) & =\frac{N_{l}}{2} \sqrt{(2 l+1) \pi} \int_{-1}^{1} \mathrm{~d} x\left(1-x^{2}\right) G_{(l m)}^{+}(x) \\
& =\frac{N_{l}}{2} \sqrt{(2 l+1) \pi} \int_{-1}^{1} \mathrm{~d} x\left[-\left(\frac{1}{2} l(l-1)\left(1-x^{2}\right)+l\right) P_{l}(x)+l x P_{l-1}(x)\right] \\
& =2 \sqrt{\frac{\pi}{15}} \delta_{l, 2},
\end{aligned}
$$

where the last line follows from orthogonality of the Legendre polynomials and the fact that we have grad modes with $l \geq 2$ only. To do the $\hat{u}_{2}$ part of the integral we can carry out a coordinate transformation to put $\hat{u}_{2}$ in the $\hat{z}$ direction (an anticlockwise rotation of $\pi / 2$ radians about the $\hat{y}$ axis). This transforms the values of the $a_{(l m)}^{P}$ coefficients. In the new frame the integral takes the same form as before, and as curl modes are transformed into curl modes, the curl response is still zero. For the grad response we need only the coefficient of $Y_{(20)}^{G}$ in the transformed coordinates. Using Eq. (89), we see this is zero for $l \neq 2$ and obtain the final result

$$
R_{(l m)}^{G}(f)=2 \sqrt{\frac{\pi}{15}} \delta_{l, 2}\left[1-\sqrt{\frac{4 \pi}{5}} Y_{2 m}(\pi / 2,0)\right]
$$

In the same way we can deduce that the response of an arbitrary two-arm detector in a frame in which the arms point in directions with spherical coordinates $\left(\theta_{1}, \phi_{1}\right)$ and $\left(\theta_{2}, \phi_{2}\right)$ is simply

$$
R_{(l m)}^{G}(f)=\frac{4 \pi}{5} \sqrt{\frac{1}{3}} \delta_{l, 2}\left(Y_{2 m}\left(\theta_{1}, \phi_{1}\right)-Y_{2 m}\left(\theta_{2}, \phi_{2}\right)\right) .
$$

If we drop the point-detector approximation but keep the vertex of the interferometer at the origin, the response function takes the form

$R^{A}(f, k)=\frac{1}{2} e_{a b}^{A}(\hat{k})\left(u_{1}^{a} u_{1}^{b} \mathcal{T}\left(f, \hat{k} \cdot \hat{u}_{1}\right)-u_{2}^{a} u_{2}^{b} \mathcal{T}\left(f, \hat{k} \cdot \hat{u}_{2}\right)\right)$,

where $\mathcal{T}$ is the transfer function. In the transformed reference frames used above, the quantities $\hat{k} \cdot \hat{u}_{1}$ and $\hat{k} \cdot \hat{u}_{2}$ depend only on the transformed polar coordinate and are independent of the transformed azimuthal coordinate. The detector therefore still has no response to curl modes, although there will now be sensitivity to grad modes with $l>2$. We leave a full treatment of the response of an extended and moving interferometer to future work. 


\section{APPENDIX E: CORRELATION CURVES FOR ANISOTROPIC UNCORRELATED BACKGROUNDS}

Expressions for the spherical harmonic components of the frequency-independent part of the pulsar timing overlap reduction function for anisotropic, unpolarized and uncorrelated backgrounds are given in [34]

$$
\bar{\Gamma}_{12, l m}=\int_{S^{2}} \mathrm{~d}^{2} \Omega_{\hat{k}} Y_{l m}(\hat{k})\left[F_{1}^{+}(\hat{k}) F_{2}^{+}(\hat{k})+F_{1}^{\times}(\hat{k}) F_{2}^{\times}(\hat{k})\right] .
$$

Analytic expressions for the quadrupole and lower terms are derived in that paper. Here we derive analytic expressions for $\bar{\Gamma}_{12, l m}$ for all values of $l$ and $m$, evaluating the integral in the computational frame, where pulsar 1 is located along the $z$ axis and pulsar 2 is located in the $x z$ plane, making an angle $\zeta$ with the $z$ axis,

$$
\begin{aligned}
& \hat{u}_{1}=(0,0,1), \\
& \hat{u}_{2}=(\sin \zeta, 0, \cos \zeta) .
\end{aligned}
$$

The calculation presented here differs from that presented in Sec. III D and in Sec. IV A, where the overlap reduction function is given by a sum of products of integrals involving the detector response functions for each pulsar separately. There we were able to evaluate the integrals over $\hat{k}$ and $\hat{k}^{\prime}$ separately by rotating coordinates so that the relevant pulsar was located along the transformed $z$ axis. Since the above expression for $\bar{\Gamma}_{12, l m}$ involves a single integral of a product of detector response functions, we can only rotate coordinates so that one pulsar is located along the transformed $z$ azis.

In this frame, we find that

$$
\begin{aligned}
F_{1}^{+}(\hat{k})= & \frac{1}{2}(1-\cos \theta), \\
F_{1}^{\times}(\hat{k})= & 0, \\
F_{2}^{+}(\hat{k})= & \frac{1}{2}[(1-\cos \zeta \cos \theta-\sin \zeta \sin \theta \cos \phi) \\
& \left.-\frac{2 \sin ^{2} \zeta \sin ^{2} \phi}{1+\cos \zeta \cos \theta+\sin \zeta \sin \theta \cos \phi}\right], \\
F_{2}^{\times}(\hat{k})= & -\frac{1}{2}\left(\frac{\sin ^{2} \zeta \cos \theta \sin (2 \phi)-\sin (2 \zeta) \sin \theta \sin \phi}{1+\cos \zeta \cos \theta+\sin \zeta \sin \theta \cos \phi}\right) .
\end{aligned}
$$

Thus, the expression for $\bar{\Gamma}_{12, l m}$ simplifies to

$$
\begin{aligned}
\bar{\Gamma}_{12, l m} & =\int_{S^{2}} \mathrm{~d}^{2} \Omega_{\hat{k}} Y_{l m}(\hat{k}) F_{1}^{+}(\hat{k}) F_{2}^{+}(\hat{k}) \\
& =\frac{1}{2} N_{l}^{m} \int_{-1}^{1} \mathrm{~d} x(1-x) P_{l}^{m}(x) I_{m}(x),
\end{aligned}
$$

where

$$
I_{m}(x) \equiv \int_{0}^{2 \pi} \mathrm{d} \phi F_{2}^{+}(\hat{k}) e^{i m \phi}
$$

The integral for $I_{m}(x)$ can be evaluated using contour integration, making the substitutions $z=e^{i \phi}, \cos \phi=$ $\left(z+z^{-1}\right) / 2$, etc. In general, the integrand has poles at 0 , $z_{+}, z_{-}$, where

$$
\begin{aligned}
z_{+} & \equiv-\frac{(1 \mp \cos \zeta)(1 \mp \cos \theta)}{\sin \zeta \sin \theta} \\
& =-\sqrt{\left(\frac{1 \mp \cos \zeta}{1 \pm \cos \zeta}\right)\left(\frac{1 \mp \cos \theta}{1 \pm \cos \theta}\right)}, \\
z_{-} & \equiv \frac{1}{z_{+}}
\end{aligned}
$$

In the above expressions, the top (bottom) sign corresponds to the region $-\cos \zeta \leq \cos \theta \leq 1 \quad(-1 \leq \cos \theta \leq-\cos \zeta)$. One can show that for both of these regions, $z_{+}$is inside the unit circle and hence contributes to the contour integral, while $z_{-}$is outside the unit circle. The results of the $\phi$ integrations for $m=0,1,2, \ldots$ are

$$
\begin{aligned}
I_{0}(x)= & \pi\left[(1-x \cos \zeta)-2 \frac{(1 \mp \cos \zeta)}{(1 \pm x)}\right], \\
I_{1}(x)= & I_{-1}(x) \\
= & \pi\left[-\frac{1}{2} \sin \zeta\left(1-x^{2}\right)^{\frac{1}{2}}+\frac{(1 \mp \cos \zeta)^{\frac{3}{2}}}{(1 \pm \cos \zeta)^{\frac{1}{2}}} \frac{(1 \mp x)^{\frac{1}{2}}}{(1 \pm x)^{\frac{3}{2}}}\right], \\
I_{m}(x)= & I_{-m}(x) \\
= & (-1)^{m+1} \pi\left[\frac{(1 \mp \cos \zeta)^{\frac{m}{2}+1}}{(1 \pm \cos \zeta)^{\frac{m}{2}}} \frac{(1 \mp x)^{\frac{m}{2}}}{(1 \pm x)^{\frac{m}{2}+1}}\right. \\
& \left.-\frac{(1 \mp \cos \zeta)^{\frac{m}{2}}}{(1 \pm \cos \zeta)^{\frac{m}{2}-1}} \frac{(1 \mp x)^{\frac{m}{2}-1}}{(1 \pm x)^{\frac{m}{2}}}\right], \quad m=2,3, \ldots .
\end{aligned}
$$

Since

$$
P_{l}^{m}(x)=(-1)^{m}(1-x)^{m / 2}(1+x)^{m / 2} \frac{\mathrm{d}^{m}}{\mathrm{~d} x^{m}} P_{l}(x),
$$

it follows that $\bar{\Gamma}_{12, l m}$ can be written in terms of sums of integrals of the form 


$$
\begin{aligned}
& \mathcal{F}_{q, r, l, m}^{-}(\cos \zeta) \equiv \int_{-1}^{-\cos \zeta} \mathrm{d} x \frac{(1+x)^{q}}{(1-x)^{r}} \frac{\mathrm{d}^{m}}{\mathrm{~d} x^{m}} P_{l}(x), \\
& \mathcal{F}_{q, r, l, m}^{+}(\cos \zeta) \equiv \int_{-\cos \zeta}^{1} \mathrm{~d} x \frac{(1-x)^{q}}{(1+x)^{r}} \frac{\mathrm{d}^{m}}{\mathrm{~d} x^{m}} P_{l}(x),
\end{aligned}
$$

where $q=m, m-1$ and $r=0,-1$ for $\mathcal{F}_{q, r, l, m}^{-}(\cos \zeta)$, and $q=m, m+1$ and $r=0,1$ for $\mathcal{F}_{q, r, l, m}^{+}(\cos \zeta)$. [The integrals for $\mathcal{F}_{q, r, l, m}^{ \pm}(\cos \zeta)$ are explicitly evaluated in the following subsection.]

Explicit expressions for $\bar{\Gamma}_{12, l m}$ are given below. For $m=0$ :

$$
\begin{aligned}
\bar{\Gamma}_{12, l 0}=\frac{\pi}{2} \sqrt{\frac{2 l+1}{4 \pi}}\left\{\int_{-1}^{1} \mathrm{~d} x(1-x)(1-x \cos \zeta) P_{l}(x)\right. \\
\left.-2(1+\cos \zeta) \int_{-1}^{-\cos \zeta} \mathrm{d} x P_{l}(x)-2(1-\cos \zeta) \int_{-\cos \zeta}^{1} \mathrm{~d} x \frac{(1-x)}{(1+x)} P_{l}(x)\right\} \\
=\frac{1}{2} \sqrt{(2 l+1) \pi}\left\{\left(1+\frac{1}{3} \cos \zeta\right) \delta_{l 0}-\frac{1}{3}(1+\cos \zeta) \delta_{l 1}+\frac{2}{15} \cos \zeta \delta_{l 2}\right. \\
\left.-(1+\cos \zeta) \mathcal{F}_{0,0, l, 0}^{-}(\cos \zeta)-(1-\cos \zeta) \mathcal{F}_{1,1, l, 0}^{+}(\cos \zeta)\right\},
\end{aligned}
$$

where we substituted combinations of Legendre polynomials for 1, $x, x^{2}$ and then used the orthogonality relation, Eq. (B6), to evaluate the integral from -1 to 1 .

Similarly for $m=1$ :

$$
\begin{aligned}
\bar{\Gamma}_{12, l 1}=\frac{\pi}{2} \sqrt{\frac{2 l+1}{4 \pi} \frac{(l-1) !}{(l+1) !}}\left\{\frac{1}{2} \sin \zeta \int_{-1}^{1} \mathrm{~d} x(1-x)\left(1-x^{2}\right) \frac{\mathrm{d}}{\mathrm{d} x} P_{l}(x)-\frac{(1+\cos \zeta)^{3 / 2}}{(1-\cos \zeta)^{1 / 2}} \int_{-1}^{-\cos \zeta} \mathrm{d} x(1+x) \frac{\mathrm{d}}{\mathrm{d} x} P_{l}(x)\right. \\
\left.\quad-\frac{(1-\cos \zeta)^{3 / 2}}{(1+\cos \zeta)^{1 / 2}} \int_{-\cos \zeta}^{1} \mathrm{~d} x \frac{(1-x)^{2}}{(1+x)} \frac{\mathrm{d}}{\mathrm{d} x} P_{l}(x)\right\} \\
=\frac{1}{4} \sqrt{(2 l+1) \pi} \sqrt{\frac{(l-1) !}{(l+1) !}}\left\{2 \sin \zeta\left(\frac{1}{3} \delta_{l 1}-\frac{1}{5} \delta_{l 2}\right)-\frac{(1+\cos \zeta)^{3 / 2}}{(1-\cos \zeta)^{1 / 2}} \mathcal{F}_{1,0, l, 1}^{-}(\cos \zeta)-\frac{(1-\cos \zeta)^{3 / 2}}{(1+\cos \zeta)^{1 / 2}} \mathcal{F}_{2,1, l, 1}^{+}(\cos \zeta)\right\}
\end{aligned}
$$

where we used integration by parts and orthogonality of Legendre polynomials, Eq. (B6), to evaluate the integral from -1 to 1 .

For $m=2,3, \cdots$ :

$$
\begin{aligned}
& \bar{\Gamma}_{12, l m}=-\frac{\pi}{2} \sqrt{\frac{2 l+1}{4 \pi} \frac{(l-m) !}{(l+m) !}}\left\{\frac{(1+\cos \zeta)^{\frac{m}{2}+1}}{(1-\cos \zeta)^{\frac{m}{2}}} \int_{-1}^{-\cos \zeta} \mathrm{dx}(1+x)^{m} \frac{\mathrm{d}^{m}}{\mathrm{~d} x^{m}} P_{l}(x)\right. \\
& -\frac{(1+\cos \zeta)^{\frac{m}{2}}}{(1-\cos \zeta)^{\frac{m}{2}-1}} \int_{-1}^{-\cos \zeta} \mathrm{dx}(1+x)^{m-1}(1-x) \frac{\mathrm{d}^{m}}{\mathrm{~d} x^{m}} P_{l}(x) \\
& \left.+\frac{(1-\cos \zeta)^{\frac{m}{2}+1}}{(1+\cos \zeta)^{\frac{m}{2}}} \int_{-\cos \zeta}^{1} \mathrm{dx} \frac{(1-x)^{m+1}}{(1+x)} \frac{\mathrm{d}^{m}}{\mathrm{~d} x^{m}} P_{l}(x)-\frac{(1-\cos \zeta)^{\frac{m}{2}}}{(1+\cos \zeta)^{\frac{m}{2}-1}} \int_{-\cos \zeta}^{1} \mathrm{dx}(1-x)^{m} \frac{\mathrm{d}^{m}}{\mathrm{~d} x^{m}} P_{l}(x)\right\} \\
& =-\frac{1}{4} \sqrt{(2 l+1) \pi} \sqrt{\frac{(l-m) !}{(l+m) !}}\left\{\frac{(1+\cos \zeta)^{\frac{m}{2}+1}}{(1-\cos \zeta)^{\frac{m}{2}}} \mathcal{F}_{m, 0, l, m}^{-}(\cos \zeta)-\frac{(1+\cos \zeta)^{\frac{m}{2}}}{(1-\cos \zeta)^{\frac{m}{2}-1}} \mathcal{F}_{m-1,-1, l, m}^{-}(\cos \zeta)\right. \\
& \left.+\frac{(1-\cos \zeta)^{\frac{m}{2}+1}}{(1+\cos \zeta)^{\frac{m}{2}}} \mathcal{F}_{m+1,1, l, m}^{+}(\cos \zeta)-\frac{(1-\cos \zeta)^{\frac{m}{2}}}{(1+\cos \zeta)^{\frac{m}{2}-1}} \mathcal{F}_{m, 0, l, m}^{+}(\cos \zeta)\right\} \text {. }
\end{aligned}
$$

For $m<0$, 


$$
\bar{\Gamma}_{12, l m}=(-1)^{m} \bar{\Gamma}_{12, l,-m},
$$

which follows from $Y_{l m}(\hat{k})=(-1)^{m} Y_{l,-m}(\hat{k})$.

Figure 8 in the main text shows plots of $\bar{\Gamma}_{12, l m}$ for $l=0,1, \ldots, 5$, calculated using the above formulas. The plots for $l=0,1,2,3$ are identical to those in Fig. 2 of [34], as expected. From the plots, we see that when the two pulsars are coaligned (i.e., when $\zeta=0$ or, equivalently, when $\hat{u}_{1}=\hat{u}_{2}$ ), the only nonzero contributions to the overlap reduction function come from $m=0$ and $l=0$, 1 , or 2 . To verify that this is the case for all $l \geq 3$, we note that in the computational frame with the coaligned pulsars located along the $z$ axis,

$$
\begin{aligned}
\bar{\Gamma}_{11, l m} & =\int_{S^{2}} \mathrm{~d}^{2} \Omega_{\hat{k}} Y_{l m}(\hat{k})\left[\left|F_{1}^{+}(\hat{k})\right|^{2}+\left|F_{1}^{\times}(\hat{k})\right|^{2}\right] \\
& =\frac{1}{4} \int_{0}^{2 \pi} \mathrm{d} \phi \int_{-1}^{1} \mathrm{~d} \cos \theta Y_{l m}(\theta, \phi)(1-\cos \theta)^{2} \\
& =\delta_{m 0} \frac{\pi}{2} \sqrt{\frac{2 l+1}{4 \pi}} \int_{-1}^{1} \mathrm{~d} x P_{l}(x)(1-x)^{2} .
\end{aligned}
$$

Since

$$
\begin{aligned}
(1-x)^{2} & =1-2 x+x^{2} \\
& =\frac{4}{3} P_{0}(x)-2 P_{1}(x)+\frac{2}{3} P_{2}(x)
\end{aligned}
$$

orthogonality of the Legendre polynomials, Eq. (B6), implies

$$
\begin{aligned}
\bar{\Gamma}_{11, l m} & =\delta_{m 0} \frac{\pi}{2} \sqrt{\frac{2 l+1}{4 \pi}}\left[\frac{8}{3} \delta_{l 0}-\frac{4}{3} \delta_{l 1}+\frac{4}{15} \delta_{l 2}\right] \\
& =\delta_{m 0}\left[\frac{\sqrt{4 \pi}}{3} \delta_{l 0}-\sqrt{\frac{\pi}{3}} \delta_{l 1}+\frac{1}{3} \sqrt{\frac{\pi}{5}} \delta_{l 2}\right]
\end{aligned}
$$

which is 0 for $l \geq 3$ or $m \neq 0$.

\section{Evaluating the $\mathcal{F}_{q, r, l, m}^{ \pm}(\cos \xi)$ integrals}

The integrals for $\mathcal{F}_{q, r, L, m}^{ \pm}(\cos \zeta)$ can be evaluated using the series representation of the Legendre polynomials

$$
P_{l}(x)=\sum_{k=0}^{l}(-1)^{k} \frac{(l+k) !}{(k !)^{2}(l-k) !}\left(\frac{1-x}{2}\right)^{k}=\sum_{k=0}^{l}(-1)^{l+k} \frac{(l+k) !}{(k !)^{2}(l-k) !}\left(\frac{1+x}{2}\right)^{k} .
$$

Explicitly, we find

$$
\begin{aligned}
\mathcal{F}_{q, r, L, m}^{-}(\cos \zeta) & \equiv \int_{-1}^{-\cos \zeta} \mathrm{d} x \frac{(1+x)^{q}}{(1-x)^{r}} \frac{\mathrm{d}^{m}}{\mathrm{~d} x^{m}} P_{L}(x) \\
& =\sum_{i=0}^{q} \sum_{j=m}^{L} 2^{i-j}(-1)^{q-i+j+m} \frac{q !(L+j) !}{i !(q-i) ! j !(L-j) !(j-m) !} \int_{-1}^{-\cos \zeta} \mathrm{d} x(1-x)^{q-i-r+j-m}
\end{aligned}
$$

for which

$$
\begin{aligned}
\mathcal{F}_{q, 0, L, m}^{-}(\cos \zeta)= & \sum_{i=0}^{q} \sum_{j=m}^{L} 2^{i-j}(-1)^{q-i+j+m} \frac{q !(L+j) !\left(2^{q-i+j-m+1}-(1+\cos \zeta)^{q-i+j-m+1}\right)}{i !(q-i) ! j !(L-j) !(j-m) !(q-i+j-m+1)} \\
\mathcal{F}_{q, 1, L, m}^{-}(\cos \zeta)= & \sum_{i=0}^{q-1} \sum_{j=m}^{L} 2^{i-j}(-1)^{q-i+j+m} \frac{q !(L+j) !\left(2^{q-i+j-m}-(1+\cos \zeta)^{q-i+j-m}\right)}{i !(q-i) ! j !(L-j) !(j-m) !(q-i+j-m)} \\
& +\sum_{j=m+1}^{L} 2^{q-j}(-1)^{j+m} \frac{(L+j) !\left(2^{j-m}-(1+\cos \zeta)^{j-m}\right)}{j !(L-j) !(j-m) !(j-m)} \\
& +\frac{2^{q-m}(L+m) !}{m !(L-m) !} \ln \left(\frac{2}{1+\cos \zeta}\right) .
\end{aligned}
$$

Similarly, 


$$
\begin{aligned}
\mathcal{F}_{q, r, L, m}^{+}(\cos \zeta) & \equiv \int_{-\cos \zeta}^{1} \mathrm{~d} x \frac{(1-x)^{q}}{(1+x)^{r}} \frac{\mathrm{d}^{m}}{\mathrm{~d} x^{m}} P_{L}(x) \\
& =\sum_{i=0}^{q} \sum_{j=m}^{L} 2^{i-j}(-1)^{L+q-i+j} \frac{q !(L+j) !}{i !(q-i) ! j !(L-j) !(j-m) !} \int_{-\cos \zeta}^{1} \mathrm{~d} x(1+x)^{q-i-r+j-m},
\end{aligned}
$$

for which

$$
\begin{aligned}
\mathcal{F}_{q, 0, L, m}^{+}(\cos \zeta)= & \sum_{i=0}^{q} \sum_{j=m}^{L} 2^{i-j}(-1)^{L+q-i+j} \frac{q !(L+j) !\left(2^{q-i+j-m+1}-(1-\cos \zeta)^{q-i+j-m+1}\right)}{i !(q-i) ! j !(L-j) !(j-m) !(q-i+j-m+1)}, \\
\mathcal{F}_{q, 1, L, m}^{+}(\cos \zeta)= & \sum_{i=0}^{q-1} \sum_{j=m}^{L} 2^{i-j}(-1)^{L+q-i+j} \frac{q !(L+j) !\left(2^{q-i+j-m}-(1-\cos \zeta)^{q-i+j-m}\right)}{i !(q-i) ! j !(L-j) !(j-m) !(q-i+j-m)} \\
& +\sum_{j=m+1}^{L} 2^{q-j}(-1)^{L+j} \frac{(L+j) !\left(2^{j-m}-(1-\cos \zeta)^{j-m}\right)}{j !(L-j) !(j-m) !(j-m)} \\
& +\frac{(-1)^{L+m} 2^{q-m}(L+m) !}{m !(L-m) !} \ln \left(\frac{2}{1-\cos \zeta}\right) .
\end{aligned}
$$

We also need to evaluate $\mathcal{F}_{q, r, l, m}^{-}(\cos \zeta)$ for $r=-1$. This can be reduced to combinations of $\mathcal{F}_{q, 0, l, m}^{-}(\cos \zeta)$ and $\mathcal{F}_{q+1,0, l, m}^{-}(\cos \zeta)$ by writing $(1-x)=2-(1+x)$,

$$
\mathcal{F}_{q,-1, l, m}^{-}(\cos \zeta)=2 \mathcal{F}_{q, 0, l, m}^{-}(\cos \zeta)-\mathcal{F}_{q+1,0, l, m}^{-}(\cos \zeta)
$$

Alternatively, we can just evaluate this integral directly, finding

$$
\mathcal{F}_{q,-1, l, m}^{-}(\cos \zeta)=\sum_{i=0}^{q} \sum_{j=m}^{l} 2^{i-j}(-1)^{q-i+j+m} \frac{q !(l+j) !\left(2^{q-i+j-m+2}-(1+\cos \zeta)^{q-i+j-m+2}\right)}{i !(q-i) ! j !(l-j) !(j-m) !(q-i+j-m+2)}
$$

[1] G. M. Harry, Classical Quantum Gravity 27, 084006 (2010).

[2] Advanced virgo baseline design, https://pub3.ego-gw.it/itf/ tds/file.php?callFile=VIR-0027 A-09.pdf.

[3] K. Somiya, Classical Quantum Gravity 29, 124007 (2012)

[4] C. S. Unnikrishnan, Int. J. Mod. Phys. D 22, 1341010 (2013).

[5] P. Amaro-Seoane et al., Classical Quantum Gravity 29, 124016 (2012).

[6] R. van Haasteren et al., Mon. Not. R. Astron. Soc. 414, 3117 (2011).

[7] P. B. Demorest et al., Astrophys. J. 762, 94 (2013).

[8] R. M. Shannon et al., Science 342, 334 (2013).

[9] R. N. Manchester and IPTA, Classical Quantum Gravity 30, 224010 (2013).

[10] A. Sesana, A. Vecchio, and M. Volonteri, Mon. Not. R. Astron. Soc. 394, 2255 (2009).

[11] K. J. Lee, N. Wex, M. Kramer, B. W. Stappers, C. G. Bassa, G. H. Janssen, R. Karuppusamy, and R. Smits, Mon. Not. R. Astron. Soc. 414, 3251 (2011).

[12] J. A. Ellis, X. Siemens, and J. D. E. Creighton, Astrophys. J. 756, 175 (2012).
[13] T. Damour and A. Vilenkin, Phys. Rev. D 64, 064008 (2001).

[14] L. Leblond, B. Shlaer, and X. Siemens, Phys. Rev. D 79, 123519 (2009).

[15] J. S. Key and N. J. Cornish, Phys. Rev. D 79, 043014 (2009).

[16] M. Rajagopal and R. W. Romani, Astrophys. J. 446, 543 (1995).

[17] A. H. Jaffe and D. C. Backer, Astrophys. J. 583, 616 (2003).

[18] J. S. B. Wyithe and A. Loeb, Astrophys. J. 590, 691 (2003).

[19] A. Vilenkin, Phys. Rev. D 24, 2082 (1981).

[20] A. Vilenkin, Phys. Lett. 107B, 47 (1981).

[21] S. Ölmez, V. Mandic, and X. Siemens, Phys. Rev. D 81, 104028 (2010).

[22] S. A. Sanidas, R. A. Battye, and B. W. Stappers, Phys. Rev. D 85, 122003 (2012).

[23] L. P. Grishchuk, J. Exp. Theor. Phys. Lett. 23, 293 (1976).

[24] L. P. Grishchuk, Phys. Usp. 48, 1235 (2005).

[25] R. S. Foster and D. C. Backer, Astrophys. J. 361, 300 (1990).

[26] M. V. Sazhin, Sov. Astron. 22, 36 (1978).

[27] S. Detweiler, Astrophys. J. 234, 1100 (1979). 
[28] F. B. Estabrook and H. D. Wahlquist, Gen. Relativ. Gravit. 6, 439 (1975).

[29] W. L. Burke, Astrophys. J. 196, 329 (1975).

[30] R. W. Hellings and G. S. Downs, Astrophys. J. 265, L39 (1983).

[31] K. J. Lee, F. A. Jenet, and R. H. Price, Astrophys. J. 685, 1304 (2008).

[32] S. J. Chamberlin and X. Siemens, Phys. Rev. D 85, 082001 (2012).

[33] K. J. Lee, Classical Quantum Gravity 30, 224016 (2013).

[34] C. M. F. Mingarelli, T. Sidery, I. Mandel, and A. Vecchio, Phys. Rev. D 88, 062005 (2013).

[35] S. R. Taylor and J. R. Gair, Phys. Rev. D 88, 084001 (2013).

[36] M. Kamionkowski, A. Kosowsky, and A. Stebbins, Phys. Rev. D 55, 7368 (1997).

[37] K. S. Thorne, Rev. Mod. Phys. 52, 299 (1980).

[38] R. Smits, M. Kramer, B. Stappers, D. R. Lorimer, J. Cordes, and A. Faulkner, Astron. Astrophys. 493, 1161 (2009).

[39] M. Maggiore, Gravitational Waves, Vol 1, Theory and Experiments (Oxford University Press, New York, 2008).

[40] A. Challinor, P. Fosalba, D. Mortlock, M. Ashdown, B. Wandelt, and K. Górski, Phys. Rev. D 62, 123002 (2000).

[41] F. J. Zerilli, Phys. Rev. D 2, 2141 (1970).

[42] W. Hu and M. White, Phys. Rev. D 56, 596 (1997).

[43] E. T. Newman and R. Penrose, J. Math. Phys. (N.Y.) 7, 863 (1966).

[44] J. N. Goldberg, A. J. MacFarlane, E. T. Newman, F. Rohrlich, and E. C. G. Sudarshan, J. Math. Phys. (N.Y.) 8, 2155 (1967).

[45] N. Christensen, Phys. Rev. D 46, 5250 (1992).

[46] É. É. Flanagan, Phys. Rev. D 48, 2389 (1993).

[47] L. S. Finn, S. L. Larson, and J. D. Romano, Phys. Rev. D 79, 062003 (2009).

[48] C. M. F. Mingarelli and T. Sidery, arXiv:1408.6840 [Phys. Rev. D (to be published)].

[49] G. B. Hobbs, R. T. Edwards, and R. N. Manchester, Mon. Not. R. Astron. Soc. 369, 655 (2006).

[50] R. T. Edwards, G. B. Hobbs, and R. N. Manchester, Mon. Not. R. Astron. Soc. 372, 1549 (2006).

[51] G. Hobbs, F. Jenet, K. J. Lee, J. P. W. Verbiest, D. Yardley, R. Manchester, A. Lommen, W. Coles, R. Edwards, and C. Shettigara, Mon. Not. R. Astron. Soc. 394, 1945 (2009).
[52] R. van Haasteren, Y. Levin, P. McDonald, and T.Lu, Mon. Not. R. Astron. Soc. 395, 1005 (2009).

[53] R. van Haasteren and Y. Levin, Mon. Not. R. Astron. Soc. 428, 1147 (2013).

[54] J. Skilling, AIP Conf. Proc. 735, 395 (2004), http://scitation .aip.org/content/aip/proceeding/aipcp/10.1063/1.1835238.

[55] F. Feroz and M. P. Hobson, Mon. Not. R. Astron. Soc. 384, 449 (2008).

[56] F. Feroz, M. P. Hobson, and M. Bridges, Mon. Not. R. Astron. Soc. 398, 1601 (2009).

[57] F. Feroz, M. P. Hobson, E. Cameron, and A. N. Pettitt, arXiv:1306.2144.

[58] E. P. Wigner, Group Theory and Its Application to the Quantum Mechanics of Atomic Spectra, expanded and improved ed. (Academic Press, New York, 1959).

[59] A. Messiah, Quantum Mechanics, Vol. 2 (North-Holland, Amsterdam, Netherlands, 1962).

[60] L. Lentati, P. Alexander, M. P. Hobson, S. Taylor, J. Gair, S. T. Balan, and R. van Haasteren, Phys. Rev. D 87, 104021 (2013).

[61] D. R. Lorimer and M. Kramer, Handbook of Pulsar Astronomy (Cambridge University Press, Cambridge, UK, 2012).

[62] N. J. Cornish and R. van Haasteren, arXiv:1406.4511.

[63] A. Sesana and A. Vecchio, Phys. Rev. D 81, 104008 (2010).

[64] N. J. Cornish and J. D. Romano, Phys. Rev. D 87, 122003 (2013).

[65] Ipta data challenge, http://www.ipta4gw.org/?page_id=89.

[66] S. R. Taylor, J. R. Gair, and L. Lentati, Phys. Rev. D 87, 044035 (2013).

[67] J. D. Jackson, Classical Electrodynamics (Wiley, New York, 1975).

[68] K. M. Górski, E. Hivon, A. J. Banday, B. D. Wandelt, F. K. Hansen, M. Reinecke, and M. Bartelmann, Astrophys. J. 622, 759 (2005).

[69] G. F. T. del Castillo, 3-D Spinors, Spin-Weighted Functions and their Applications (Springer, New York, 2003).

[70] L. D. Landau and E. M. Lifshitz, Quantum Mechanics: Non-relativistic Theory 3rd ed. (Butterworth-Heinemann, Oxford, UK, 1977).

[71] M. Abramowitz and I. A. Stegun, Handbook of Mathematical Functions (Dover, New York, 1972). 